\title{
C2-Selective Direct Alkynylation of Indoles
}

\author{
Gergely L. Tolnai, Stephanie Ganss, ${ }^{\dagger}$ Jonathan P. Brand and \\ Jérôme Waser*
}

Laboratory of Catalysis and Organic Synthesis, Institute of Chemical Sciences and

Engineering, Ecole Polytechnique Fédérale de Lausanne, EPFL SB ISIC LCSO, BCH

4306, 1015 Lausanne, Switzerland.

jerome.waser@epfl.ch

Received Date (will be automatically inserted after manuscript is accepted)

ABSTRACT

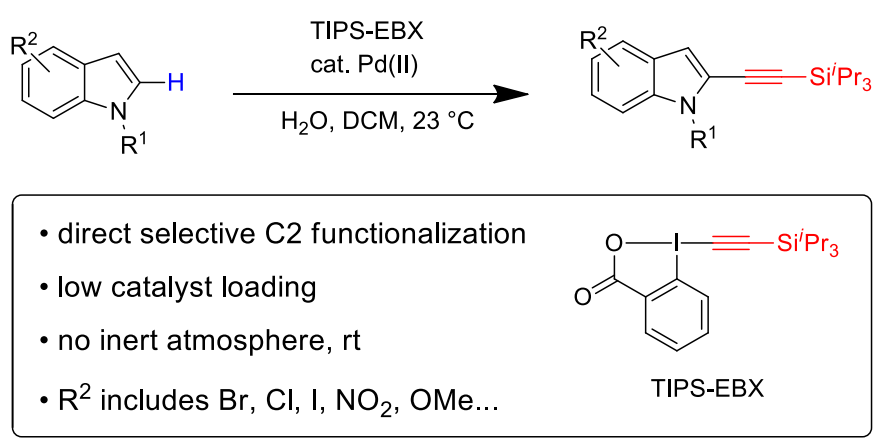

The first C2-selective alkynylation of indoles using the hypervalent iodine reagent triisopropylsilylethynyl-1,2benziodoxol-3(1H)-one (TIPS-EBX) with $\mathrm{Pd}(\mathrm{II})$ as a catalyst is described. This convenient and robust method gives a single-step access to substituted alkynyl indoles with very high C2 selectivity. The reaction is orthogonal to classical $\mathrm{Pd}(0)$ cross-coupling reactions as it is tolerant to bromide and iodide substituents. The used silyl protecting group can be easily removed to give terminal acetylenes.

Since the first synthesis of indole by Baeyer almost 150 years ago, ${ }^{1}$ interest in the preparation and functionalization of this privileged heterocycle has constantly grown. ${ }^{2}$ Indoles can indeed be found in numerous important molecules such as pharmaceuticals, dyes and natural products. Consequently, methods to synthesize and modify this heterocycle are of utmost importance in organic chemistry.

\footnotetext{
New Address: Institut für Organische Chemie und Biochemie, Albert-Ludwigs-Universität, Freiburg, Germany.

(1) Baeyer, A. Liebigs. Ann. Chem. 1866, 140, 295.

(2) (a) Sundberg, R.J. Indoles; Academic: New York, USA, 1996. (b) Cacchi, S.; Fabrizi, G. Chem. Rev. 2005, 105, 2873. (c) Humphrey, G. R.; Kuethe, J. T. Chem. Rev. 2006, 106, 2875. (d) Bandini, M.; Eichholzer, A. Angew. Chem., Int. Ed. 2009, 48, 9608. (e) Cacchi, S.; Fabrizi, G. Chem. Rev. 2011, 111, PR215-PR283.
}

Metal-catalyzed cross-coupling reactions constitute an efficient tool for the modification of aromatic rings, ${ }^{3}$ but the need for pre-functionalization makes this method less efficient. In comparison, $\mathrm{C}-\mathrm{H}$ functionalization constitutes a more direct alternative for the introduction of various valuable functional groups. Recently, the direct functionalization of indoles has been intensively examined using metal catalysts to complement traditional Friedel-Crafts reactions. Efficient methods have been developed to introduce vinyl $l^{4}$, aryl, ${ }^{5}$ alkyl $^{6}$ and cyano ${ }^{7}$

(3) de Meijere, A.; Diedrich, F. Metal-Catalyzed Cross-Coupling Reactions, 2nd, Completely Revised and Enlarged Edition; WileyWCH: Weinheim, Germany, 2004.

(4) Selected examples: (a) Grimster, N. P.; Gauntlett, C.; Godfrey, C. R. A.; Gaunt, M. J. Angew. Chem., Int. Ed. 2005, 44, 3125. (b) GarciaRubia, A.; Arrayas, R. G.; Carretero, J. C. Angew. Chem., Int. Ed. 2009, 48, 6511. (c) Ding, Z. H.; Yoshikai, N. Angew. Chem., Int. Ed. 2012, 51, 
groups among others. ${ }^{8}$ In several cases, the $\mathrm{C} 2 / \mathrm{C} 3$ regioselectivity of these functionalizations could be controlled by the reaction conditions or using directing groups. ${ }^{4,9}$

Despite the important applications of acetylenes in synthetic chemistry, biochemistry, and material sciences, ${ }^{10}$ there are only a few methods for the direct alkynylation of the indole core. ${ }^{11}$ In 2009, Gu and Wang first introduced the $\mathrm{C} 3$-selective alkynylation of indoles using bromoacetylenes and a Pd catalyst. ${ }^{1 \text { a }} \mathrm{C}$-selective alkynylation is especially challenging and only two examples have been reported so far. Li and co-workers described an oxidative Heck-Cassar-Sonogashira type method for the alkynylation of 1,3-dimethylindole. ${ }^{11 \mathrm{f}}$ This reaction could be applied to a broad scope of acetylenes, but only 3-methylindoles were reported. More recently, a method for the alkynylation of lithiated indoles using ethynylsulfonates as reagents was reported by Garcia Ruano and co-workers. ${ }^{1 \mathrm{~g}, \mathrm{~h}}$ Depending of the sterical hinderance of the substituent on the indole nitrogen, $\mathrm{C} 2$ or C3 alkynylation could be obtained. Nevertheless, the requirement for a strong base such as butyl lithium limited the scope of this transformation. Consequently, the most frequently used methods to access 2 -alkynylated indoles are often based on the formation of the heterocycles via cyclisation reactions. ${ }^{12}$

In 2009, our group introduced the hypervalent iodine compound triisopropylsilylethynyl-1,2-benziodoxol-

4698. (d) Kandukuri, S. R.; Schiffner, J. A.; Oestreich, M. Angew. Chem., Int. Ed. 2012, 51, 1265.

(5) Selected examples: (a) Lane, B. S.; Sames, D. Org Lett 2004, 6, 2897. (b) Wang, X.; Lane, B. S.; Sames, D. J. Am. Chem. Soc. 2005 , 127, 4996. (c) Lane, B. S.; Brown, M. A.; Sames, D. J. Am. Chem. Soc. 2005, 127, 8050. (d) Deprez, N. R.; Kalyani, D.; Krause, A.; Sanford, M. S. J. Am. Chem. Soc. 2006, 128, 4972. (e) Stuart, D. R.; Fagnou, K. Science 2007, 316, 1172. (f) Lebrasseur, N.; Larrosa, I. J. Am. Chem. Soc. 2008, 130, 2926. (g) Phipps, R. J.; Grimster, N. P.; Gaunt, M. J. J. Am. Chem. Soc. 2008, 130, 8172. (h) Cornella, J.; Lu, P. F.; Larrosa, I. Org. Lett. 2009, 11, 5506. (i) Potavathri, S.; Pereira, K. C.; Gorelsky, S. I.; Pike, A.; LeBris, A. P.; DeBoef, B. J. Am. Chem. Soc. 2010, 132 , 14676

(6) Jiao, L.; Bach, T. J. Am. Chem. Soc. 2011, 133, 12990.

(7) (a) Yan, G. B.; Kuang, C. X.; Zhang, Y.; Wang, J. B. Org. Lett. 2010, 12, 1052. (b) Xu, S.; Huang, X.; Hong, X.; Xu, B. Org. Lett 2012, $14,4614$.

(8) Reviews: (a) Beck, E. M.; Gaunt, M. J., Pd-Catalyzed C-H Bond Functionalization on the Indole and Pyrrole Nucleus. In $\mathrm{C}-\mathrm{H}$ Activation, Yu, J. Q.; Shi, Z., Eds. Springer-Verlag Berlin: Berlin, 2010; Vol. 292, pp 85-121. (b) Lebrasseur, N.; Larrosa, I., Recent Advances in the C2 and C3 Regioselective Direct Arylation of Indoles. In Advances in Heterocyclic Chemistry, Vol 105, Katritzky, A. R., Ed. Elsevier Academic Press Inc: San Diego, 2012; Vol. 105, pp 309-351.

(9) Huestis, M. P.; Chan, L.; Stuart, D. R.; Fagnou, K. Angew Chem, Int. Ed. 2011, 50, 1338 .

(10) Diedrich, F.; Stang, P. J.; Tykwinsky, R. R. Acetylene Chemistry; Wiley-WCH: Weinheim, Germany, 2004.

(11) (a) Gu, Y. H.; Wang, X. M. Tetrahedron Lett. 2009, 50, 763. (b) Brand, J. P.; Charpentier, J.; Waser, J. Angew. Chem., Int. Ed. 2009, 48, 9346. (c) Brand, J. P.; Chevalley, C.; Waser, J. Beilstein J. Org. Chem. 2011, 7, 565. (d) Brand, J. P.; Chevalley, C.; Scopelliti, R.; Waser, J. Chem. Eur. J. 2012, 18, 5655. (e) de Haro, T.; Nevado, C. J. Am. Chem. Soc. 2010, 132, 1512. (f) Yang, L.; Zhao, L.; Li, C. J. Chem. Commun. 2010, 46, 4184. (g) García Ruano, J. L.; Alemán, J.; Marzo, L.; Alvarado, C.; Tortosa, M.; Díaz-Tendero, S.; Fraile, A. Angew. Chem., Int. Ed. 2012, 51, 2712. (h) García Ruano, J. L.; Alemán, J.; Marzo, L.; Alvarado, C.; Tortosa, M.; Díaz-Tendero, S.; Fraile, A. Chem. Eur. J. 2012, 18,8414 .

(12) Mothe, S. R.; Kothandaraman, P.; Lauw, S. J.; Chin, S. M.; Chan, P. W. Chem. Eur J. 2012, 18, 6133. And references herein.
3(1H)-one (TIPS-EBX, 2) $)^{13}$ as an efficient reagent for the gold-catalyzed C3 alkynylation of indoles (Scheme 1). During our first investigation, palladium catalysts gave only traces of product, albeit with very high $\mathrm{C} 2$ selectivity. ${ }^{1 \mathrm{~b}}$ We later demonstrated that efficient acetylene transfer with Pd catalysts was possible for the amino- and oxy- alkynylation of olefins. ${ }^{14}$ Building upon these results, we report herein the first Pd-catalyzed C2selective alkynylation of 3H-indoles using TIPS-EBX (2), which proceeds at room temperature under air in presence of a broad range of functional groups (Scheme 1). In contrast to $\mathrm{Gu}$ and Wang's work, exclusive $\mathrm{C} 2$ alkynylation was observed. To the best of our knowledge, our work constitutes also the first example of Pdcatalyzed direct alkynylation of a heterocycle using a hypervalent iodine reagent.

Scheme 1. Regioselective alkynylation of indoles.

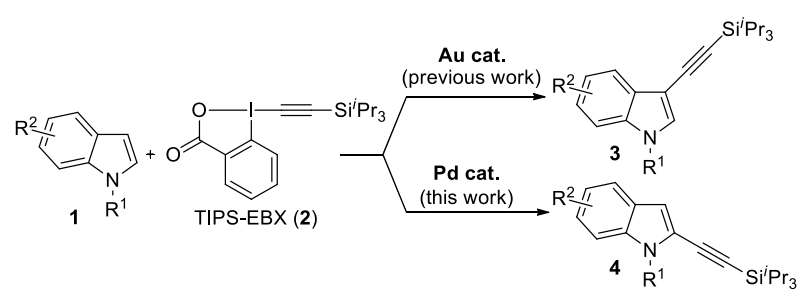

During preliminary investigations on indole itself, a broad screen of Pd catalysts, solvents and reaction conditions was not successful to improve the yield beyond $20 \%$. More promising results were obtained in the case of N-methyl indole (1a) using a dichloromethane/water mixture as solvent and three equivalents of TIPS-EBX (2) (Table 1). ${ }^{15}$ In this case, the reaction did not proceed without catalyst (entry 1) or with the $\operatorname{Pd}(0)$ source $\mathrm{Pd}\left(\mathrm{PPh}_{3}\right)_{4}$ (entry 2), but $\mathrm{Pd}(\mathrm{II})$ salts such as $\mathrm{Pd}(\mathrm{OAc})_{2}$ and $\mathrm{PdCl}_{2}$ gave promising yields (entries 3 and 4). A further increase in yield was observed with $\mathrm{Pd}(\mathrm{MeCN})_{4}\left(\mathrm{BF}_{4}\right)_{2}$, which has a less coordinating counteranion (entry 5). In this case, the importance of water was confirmed, as a lower yield was obtained under dry conditions (entry 6). The catalyst loading had a strong influence on the yield, with $2 \%$ being the optimal amount (entries 7-9). Further screening of catalysts did not lead to better yields and confirmed that the reaction did not proceed in the presence of phosphine ligands (entries 1012). When the reaction was scaled up to $0.5 \mathrm{mmol}$, the

(13) TIPS-EBX is commercially avaible, and easily preparable in a two step protocol from iodobenzoic acid in high yield on a $30 \mathrm{~g}$ scale: Brand, J. P.; Waser, J. Synthesis 2012, 44, 1155.

(14) (a) Nicolai, S.; Erard, S.; Fernandez Gonzalez, D.; Waser, J. Org. Lett. 2010, 12, 384. (b) Nicolai, S.; Piemontesi, C.; Waser, J. Angew. Chem., Int. Ed. 2011, 50, 4680.

(15) A broad range of other solvents and additives were examined, but without positive effect on the reaction outcome. A steady improvement of the yield was observed with increasing amounts of TIPS-EBX (2) up to three equivalents. Other silyl substituted alkynes gave lower yields and no product was observed with aryl or alkyl substituted acetylenes. See Supporting Information for details. 
alkynylation product was obtained in $66 \%$ isolated yield with $\mathrm{Pd}(\mathrm{MeCN})_{4}\left(\mathrm{BF}_{4}\right)_{2}$ (entry 13). In contrast to the work of $\mathrm{Gu}$ and Wang, ${ }^{11 \mathrm{a}}$ only the $\mathrm{C} 2$-alkynylated product was isolated from the reaction mixture. This transformation consequently was the first $\mathrm{C} 2$-selective direct alkynylation of 3-unsubstituted indoles.

Table 1. Optimization of the $\mathrm{C} 2$ selective alkynylation.

\begin{tabular}{|c|c|c|c|}
\hline entry & catalyst loading & Pd source & $\operatorname{yield}(\%)^{\mathrm{a}}$ \\
\hline 1 & & - & 0 \\
\hline 2 & $10 \%$ & $\mathrm{Pd}\left(\mathrm{PPh}_{3}\right)_{4}$ & 0 \\
\hline 3 & $10 \%$ & $\mathrm{Pd}(\mathrm{OAc})_{2}$ & 34 \\
\hline 4 & $10 \%$ & $\mathrm{PdCl}_{2}$ & 40 \\
\hline 5 & $10 \%$ & $\mathrm{Pd}(\mathrm{MeCN})_{4}\left(\mathrm{BF}_{4}\right)_{2}$ & 50 \\
\hline 6 & $10 \%$ & $\mathrm{Pd}(\mathrm{MeCN})_{4}\left(\mathrm{BF}_{4}\right)_{2}$ & $23^{\mathrm{b}}$ \\
\hline 7 & $25 \%$ & $\mathrm{Pd}(\mathrm{MeCN})_{4}\left(\mathrm{BF}_{4}\right)_{2}$ & 19 \\
\hline 8 & $0.5 \%$ & $\mathrm{Pd}(\mathrm{MeCN})_{4}\left(\mathrm{BF}_{4}\right)_{2}$ & 37 \\
\hline 9 & $2 \%$ & $\mathrm{Pd}(\mathrm{MeCN})_{4}\left(\mathrm{BF}_{4}\right)_{2}$ & 61 \\
\hline 10 & $2 \%$ & {$[\mathrm{Pd}(\text { allyl }) \mathrm{Cl}]_{2}$} & 60 \\
\hline 11 & $2 \%$ & $\mathrm{Pd}\left(\mathrm{PPh}_{3}\right)_{2} \mathrm{Cl}_{2}$ & traces \\
\hline 12 & $2 \%$ & $\mathrm{Pd}_{2} \mathrm{dba}_{3}$ & 57 \\
\hline 13 & $2 \%$ & $\mathrm{Pd}(\mathrm{MeCN})_{4}\left(\mathrm{BF}_{4}\right)_{2}$ & $66^{c}$ \\
\hline
\end{tabular}

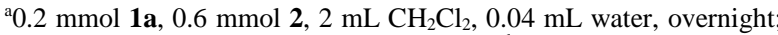
GC-MS yields, using dodecanitrile as standard. ${ }^{\text {IIn }}$ dry $\mathrm{CH}_{2} \mathrm{Cl}_{2}$. ${ }^{\mathrm{C}}$ Isolated yield on a $0.5 \mathrm{mmol}$ scale.

The reaction worked well with different halogens on various positions on the benzene ring (Table 2, entries 27), which has two main advantages. First, halogen substituents can be used to adjust the polarity, liphophilicity and metabolic stability of dyes or pharmaceuticals. Second, halogens allow further modification using cross-coupling reactions for the elaboration of molecule libraries. These results also indicated that the reaction most likely did not proceed via a $\operatorname{Pd}(0)$ intermediate, as oxidative addition on the carbonhalogen bond would have been expected in this case. This is an important advantage when compared with previously published methods involving $\operatorname{Pd}(0)$ catalysis. $^{11 \mathrm{a}}$ Furthermore, both electron-withdrawing (entries 8-9) and electron-donating (entry 10) groups were tolerated on the benzene ring.

In general, $\mathrm{N}$-alkylated indoles are very important building blocks for the synthesis of bioactive compounds, in particular for natural indole alkaloids. We consequently decided to further examine the scope of alkyl groups on the nitrogen (Table 3). ${ }^{16}$ Propylphenyl substituted indole $1 \mathbf{k}$ gave $58 \%$ yield, demonstrating that the reaction was not limited to the small methyl group (entry 1). Allyl or

(16) The required substrates were synthesized using known procedures: (a) Guida, W. C.; Mathre, D. J. J. Org. Chem. 1980, 45, 3172. (b) Ottoni, O.; Cruz, R.; Alves, R. Tetrahedron 1998, 54, 13915 (c) Bode, J. W.; Carreira, E. M. J. Org. Chem. 2001, 66, 6410. (d) Bressy, C.; Alberico, D.; Lautens, M. J. Am. Chem. Soc. 2005, 127, 13148. (e) Jorapur, Y. R.; Jeong, J. M.; Chi, D. Y. Tetrahedron Lett. 2006, 47, 2435. See Supporting Information for further details. benzyl groups on nitrogen could present a serious issue in the presence of a palladium catalyst. Nevertheless, the alkynylation products could still be obtained in moderate yields in this case (entries 2-4). Conversely, a TIPSOethyl and a sensitive bromo ethyl groups gave good yields, opening the door for a wide range of further synthetic modifications (entries 5-6). Finally, indole 1q, bearing an acetal protected aldehyde, could also be alkynylated in $66 \%$ yield (entry 7 ).

Table 2. Scope of substituents on the benzene ring.

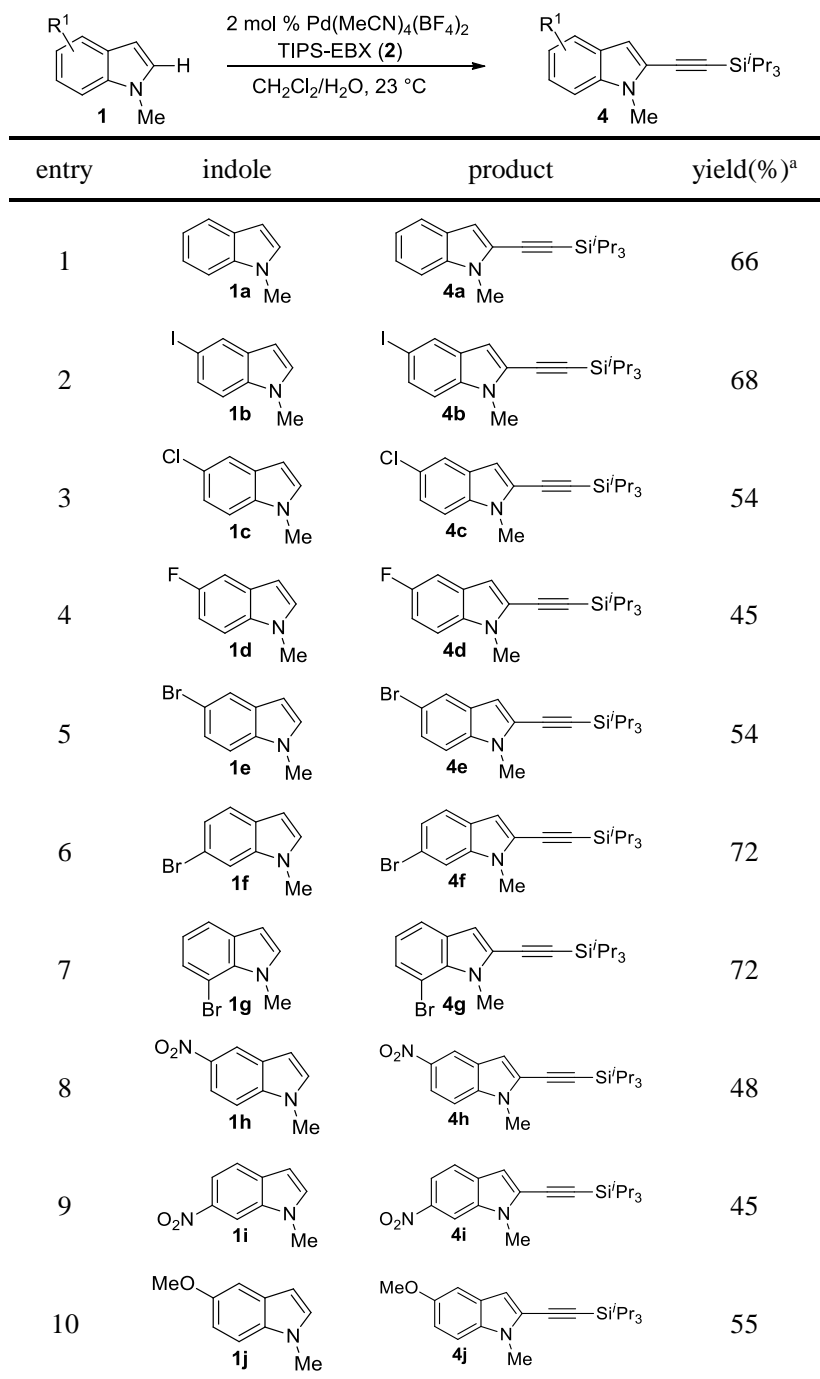

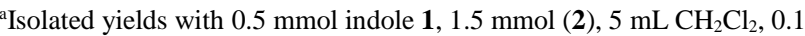
$\mathrm{mL}$ water, $10 \mu \mathrm{mol} \mathrm{Pd}(\mathrm{MeCN})_{4}\left(\mathrm{BF}_{4}\right)_{2}$.

Based on precedence from other $\mathrm{Pd}$-mediated transformations, in particular arylation, ${ }^{5}$ a speculative mechanism may possibly involve a $\mathrm{Pd}(\mathrm{II}) /(\mathrm{IV})$ cycle (Scheme 2). The reaction could be initiated by a $\mathrm{C} 2-$ palladation to give intermediate II, either via direct concerted metallation-deprotonation (CMD, path a) ${ }^{5 i, 17}$ or via electrophilic palladation at the $\mathrm{C} 3$ position to give $\mathbf{I}$,

(17) Lafrance, M.; Fagnou, K. J. Am. Chem. Soc. 2006, 128, 16496. 
followed by Pd migration (path b) ${ }^{5 \mathrm{~d}, 18}$ Water or waterclusters could play a key role in promoting this metallation-deprotonation step. The indole Pd complex II can then be oxidatively alkynylated by TIPS-EBX (2), to give $\mathrm{Pd}(\mathrm{IV})$ intermediate $\mathbf{I I I},{ }^{19}$ which undergoes reductive elimination to give the product $4 \mathbf{a}$ and regenerate the $\mathrm{Pd}(\mathrm{II})$ catalyst.

Table 3. Scope of substituents on the nitrogen.

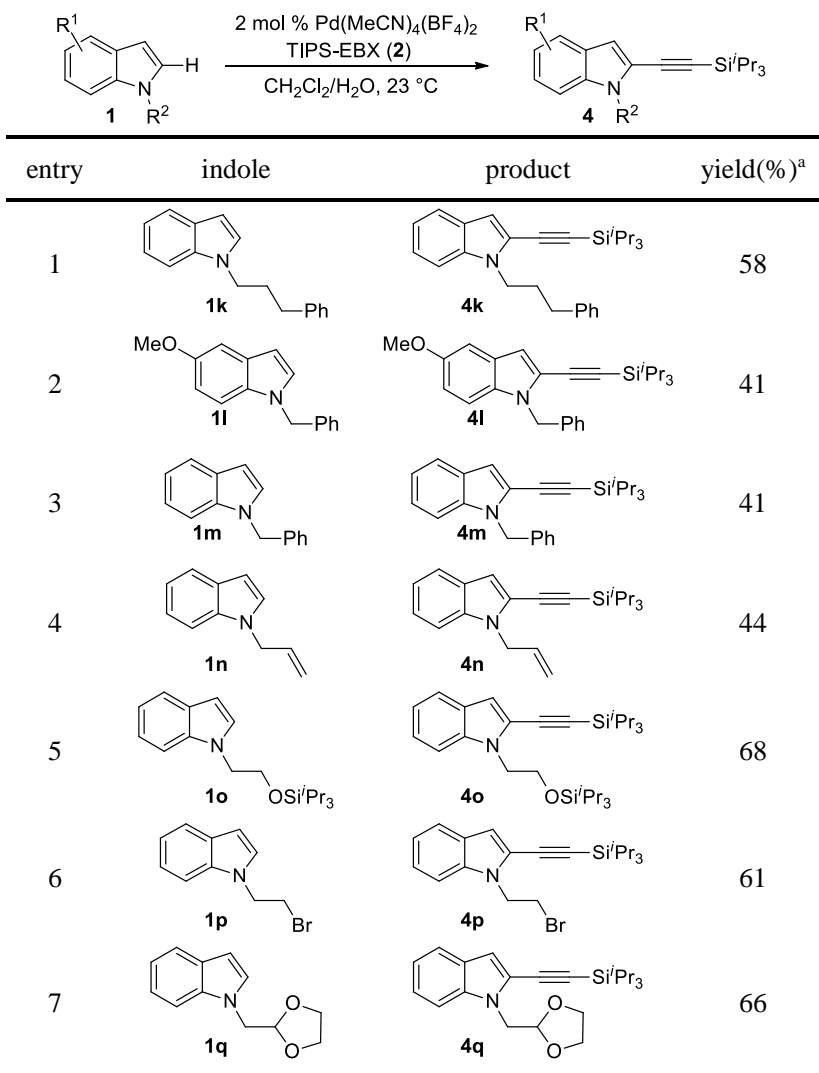

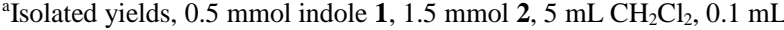
water, $10 \mu \mathrm{mol} \mathrm{Pd}(\mathrm{MeCN})_{4}\left(\mathrm{BF}_{4}\right)_{2}$.

In conclusion, we have described the first Pd-catalyzed alkynylation of indoles proceeding with high $\mathrm{C} 2$ regioselectivity. The reaction is orthogonal to classical cross-coupling reactions, and has a wide range of functional group tolerance both on the indole core and the alkyl substituent on the nitrogen. The mild and neutral reaction conditions and the tolerance of the process towards air and moisture lead to a convenient method for the direct $\mathrm{C} 2$ alkynylation of indoles.

(18) Li, Y.; Wang, W.-H.; He, K.-H.; Shi, Z. J. Organometallics 2012, 31, 4397 .

(19) (a) Canty, A. J.; Rodemann, T.; Skelton, B. W.; White, A. H. Organometallics 2006, 25, 3996. For a recent review on high valent metal catalysis, see: (b) Hickman, A. J.; Sanford, M. S. Nature 2012, 484,177 . The involvement of $\operatorname{Pd}(\mathrm{IV})$ or Pd(III) dimers in catalytic cycles is currently a topic of intensive discussion, see for example: (c) Powers, D. C.; Ritter, T. Nat. Chem. 2009, 1, 302. (d) Powers, D. C.; Lee, E.; Ariafard, A.; Sanford, M. S.; Yates, B. F.; Canty, A. J.; Ritter, T. J. Am. Chem. Soc. 2012, 134, 12002.
Scheme 2. Speculative mechanism for the alkynylation reaction.

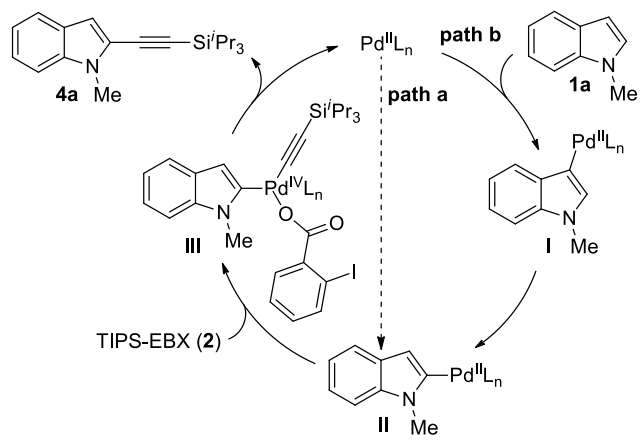

Supporting Information. Experimental procedures and analytical data for all new compounds. This material is available free of charge via the Internet at http://pubs.acs.org.

Acknowlegment. We thank the EPFL for funding and F. Hoffmann-La Roche Ltd for an unrestricted research grant. The work of G. L. T. was supported by a SciexNMS $^{\text {ch }}$ fellowship of the Swiss confederation. We thank Dr. Reto Frei (LCSO, EPFL) for proofreading the manuscript. 


\title{
C2 Selective Direct Alkynylation of Indoles
}

\author{
Gergely L. Tolnai, Stephanie Ganss, Jonathan P. Brand and Jérôme Waser
}

Laboratory of Catalysis and Organic Synthesis, Institute of Chemical Sciences and Engineering, Ecole Polytechnique Fédérale de Lausanne, EPFL SB ISIC LCSO, BCH 4306, 1015 Lausanne, Switzerland.

jerome.waser@epfl.ch 


\section{Contents}

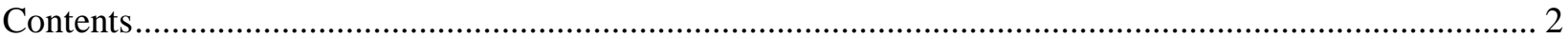

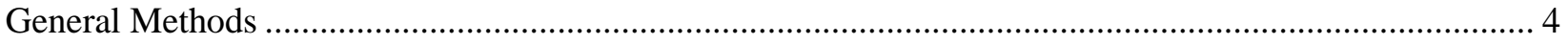

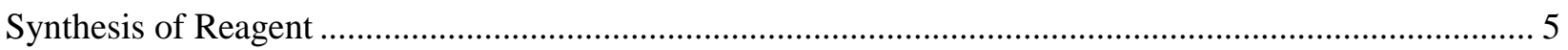

Synthesis of tetrakis(acetonitrile)palladium(II)tetrafluoroborate ….................................................... 7

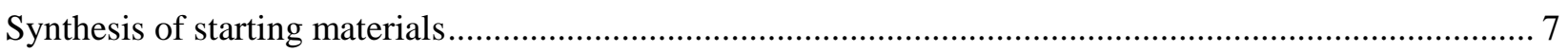

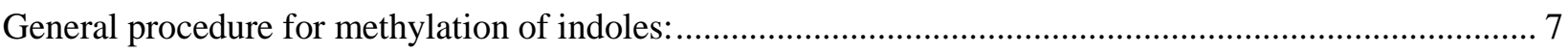

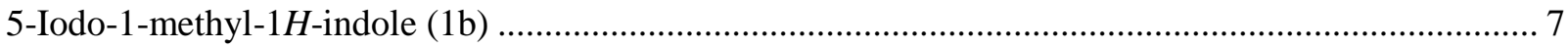

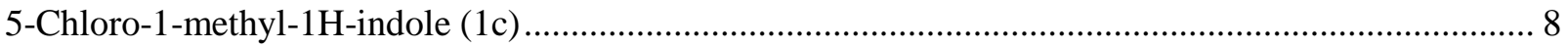

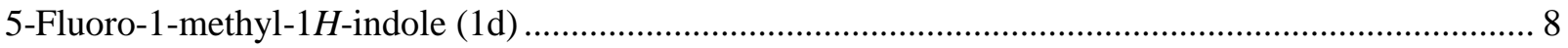

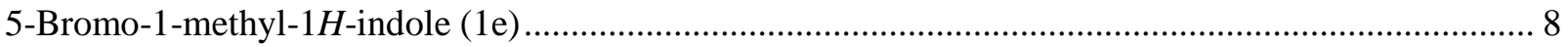

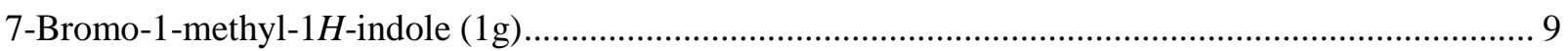

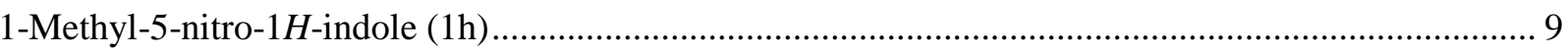

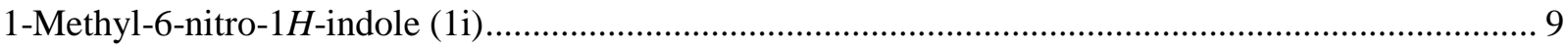

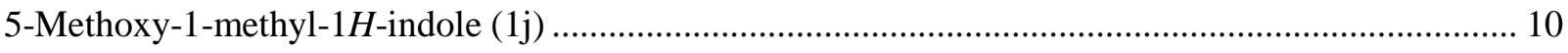

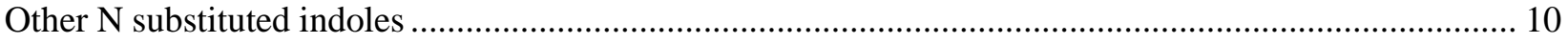

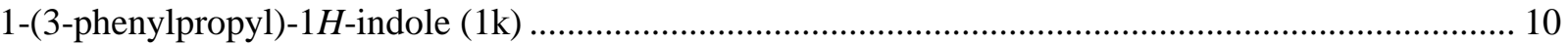

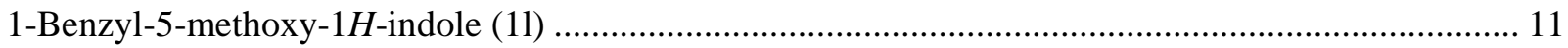

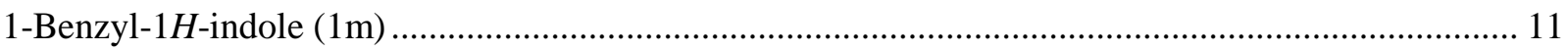

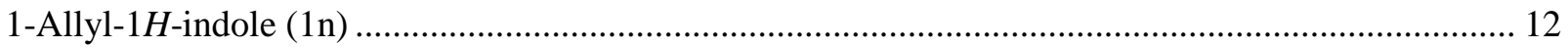

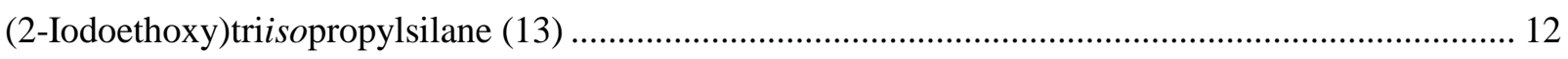

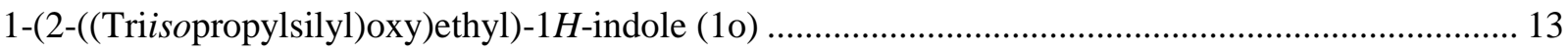

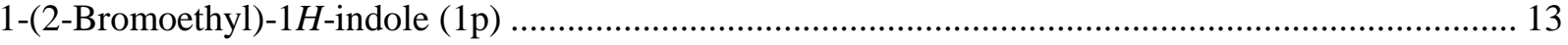

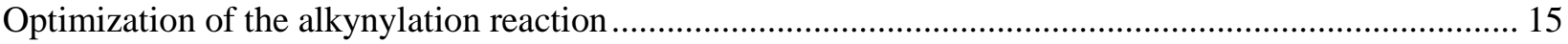

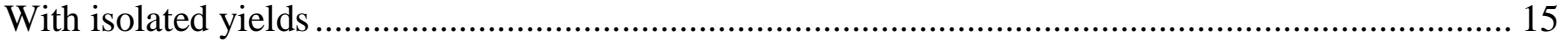

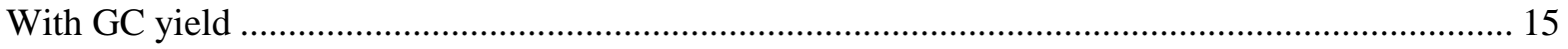

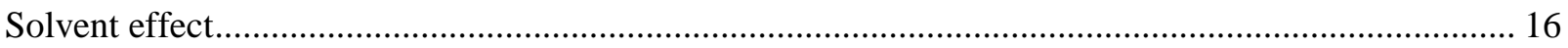

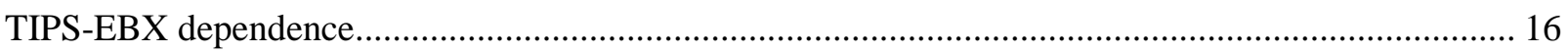

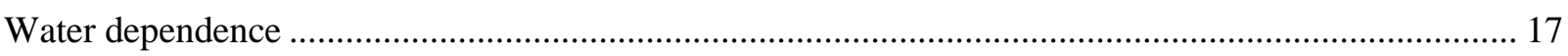

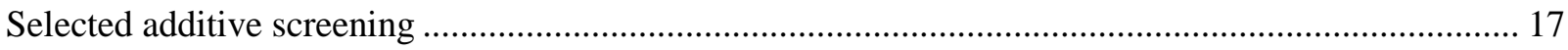

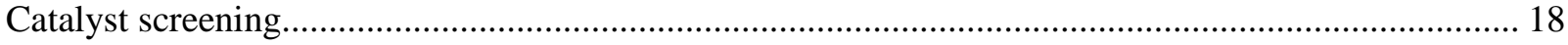

General procedure for $\mathrm{C} 2$ selective alkynylation of indoles. ............................................................ 18

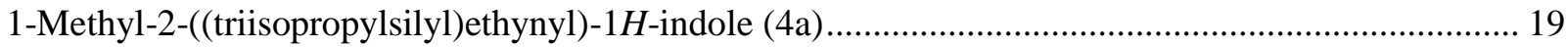


5-Iodo-1-methyl-2-((triisopropylsilyl)ethynyl)- $1 H$-indole (4b)

5-Chloro-1-methyl-2-((triisopropylsilyl)ethynyl)-1H-indole (4c)

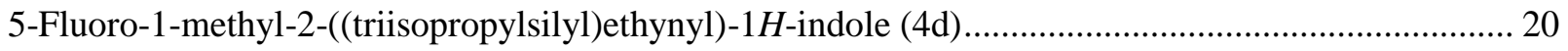

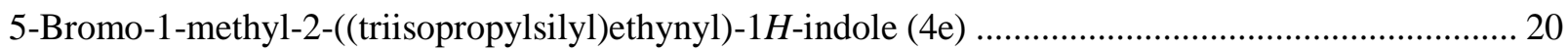

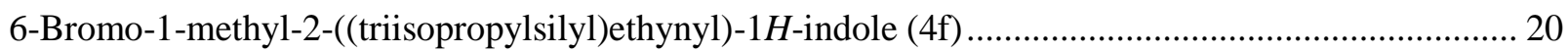

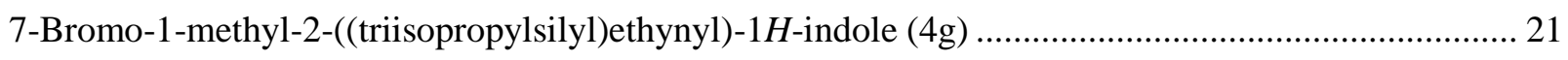

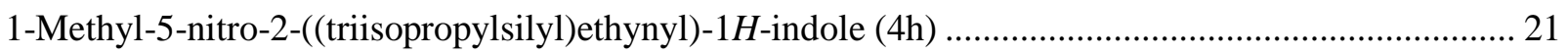

1-Methyl-6-nitro-2-((triisopropylsilyl)ethynyl)-1 $H$-indole (4i) ...................................................... 21

5-Methoxy-1-methyl-2-((triisopropylsilyl)ethynyl)-1 $H$-indole (4j) ................................................... 22

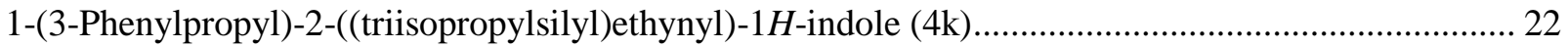

1-Benzyl-5-methoxy-2-((triisopropylsilyl)ethynyl)-1 $H$-indole (4l) .................................................. 22

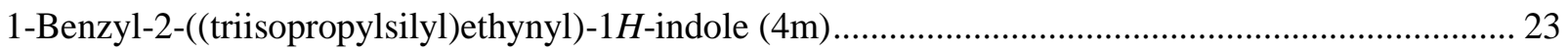

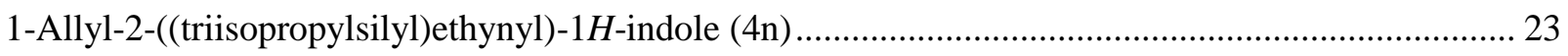

2-((Triisopropylsilyl)ethynyl)-1-(2-((triisopropylsilyl)oxy)ethyl)- $1 H$-indole (4o) ............................. 23

1-(2-Bromoethyl)-2-((triisopropylsilyl)ethynyl)-1 $H$-indole (4p) ................................................... 24

1-((1,3-Dioxolan-2-yl)methyl)-2-((triisopropylsilyl)ethynyl)-1 $H$-indole (4q) .................................... 24

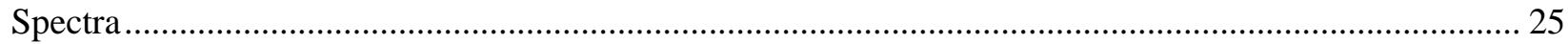




\section{General Methods}

All reactions were carried out in oven dried glassware under an atmosphere of nitrogen, unless stated otherwise. For quantitative flash chromatography technical grade solvents were used. For flash chromatography for analysis, HPLC grade solvents from Sigma-Aldrich were used. THF, $\mathrm{Et}_{2} \mathrm{O}, \mathrm{CH}_{3} \mathrm{CN}$, toluene, hexane and $\mathrm{CH}_{2} \mathrm{Cl}_{2}$ were dried by passage over activated alumina under nitrogen atmosphere $\left(\mathrm{H}_{2} \mathrm{O}\right.$ content $<10 \mathrm{ppm}$, Karl-Fischer titration). All chemicals were purchased from Acros, Aldrich, Fluka, VWR, Fluorochem, Aplichem or Merck and used without further purification, unless stated otherwise. Chromatographic purification was performed as flash chromatography using Macherey-Nagel silica 40-63, $60 \AA$, using the solvents indicated as eluent with 0.1-0.5 bar pressure. TLC was performed on Merck silica gel $60 \mathrm{~F}_{254}$ TLC aluminium plates and visualized with UV light and anisaldehyde stain. Melting points were measured on a calibrated Büchi B-540 melting point apparatus using open glass capillaries. ${ }^{1} \mathrm{H}-\mathrm{NMR}$ spectra were recorded on a Bruker DPX-400 $400 \mathrm{MHz}$ spectrometer in chloroform-d and/or DMSO- $\mathrm{d}_{6}$. All signals are reported in ppm with the internal chloroform signal at $7.26 \mathrm{ppm}$ or the internal DMSO signal at $2.50 \mathrm{ppm}$ as standard. The data is being reported as $(\mathrm{s}=$ singlet, $\mathrm{d}=$ doublet, $\mathrm{t}=$ triplet, $\mathrm{q}=$ quadruplet, $\mathrm{qi}=$ quintet, $\mathrm{m}=$ multiplet or unresolved, $\mathrm{br}=$ broad signal, app = apparent, coupling constant(s) in $\mathrm{Hz}$, integration, interpretation) ${ }^{13} \mathrm{C}-\mathrm{NMR}$ spectra were recorded with ${ }^{1} \mathrm{H}$ decoupling on a Bruker DPX-400 $100 \mathrm{MHz}$ spectrometer in chloroform-d and/or DMSO-d 6 . All signals are reported in ppm with the internal chloroform signal at $77.0 \mathrm{ppm}$ or the internal DMSO signal at 39.5 ppm as standard. Infrared spectra were recorded on a JASCO FT-IR B4100 spectrophotometer with an ATR PRO410-S and a ZnSe prisma and are reported as $\mathrm{cm}^{-1}(\mathrm{w}=$ weak, $\mathrm{m}=$ medium, $\mathrm{s}=$ strong, br $=$ broad). Gas chromatographic and low resolution mass spectrometric measurements were performed on a Perkin-Elmer Clarus 600 gas chromatographer and mass spectrometer using a Perkin-Elmer Elite fused silica column (length: $30 \mathrm{~m}$, diameter: $0.32 \mathrm{~mm}$ ) and Helium as carrier gas. High resolution mass spectrometric measurements were performed by the mass spectrometry service of ISIC at the EPFL on a MICROMASS (ESI) Q-TOF Ultima API. 


\section{Synthesis of Reagent}

\section{1-Hydroxy-1,2-benziodoxol-3(1H)-one (6)}

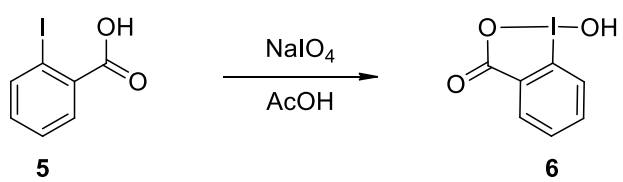

Caution: This reaction should be carried out behind a safety shield! Following a reported procedure ${ }^{1}, \mathrm{NaIO}_{4}(77.2 \mathrm{~g}, 361 \mathrm{mmol}, 1.0$ equiv) and 2-iodobenzoic acid (5) (89.5 g, $361 \mathrm{mmol}$, 1.0 equiv) were suspended in $30 \%$ (v:v) aq. $\mathrm{AcOH}(700 \mathrm{~mL})$ under air in a 4-neck sulfonation flask equipped with a mechanic stirrer, a thermometer and a condenser. The mixture was vigorously stirred and refluxed for $4 \mathrm{~h}$. The reaction mixture was then diluted with cold water $(500 \mathrm{~mL})$ and allowed to cool to room temperature, protecting it from light. After $45 \mathrm{~min}$, the suspension was added to water $(1.5 \mathrm{~L})$ and the crude product was collected by filtration, washed on the filter with ice cold water $(3 \times 300 \mathrm{~mL})$ and cold acetone $(3 \times 300 \mathrm{~mL})$, and air-dried in the dark overnight to give the pure product 1-hydroxy-1,2-benziodoxol-3(1H)-one (6) $(77.3 \mathrm{~g}, 0.292$ mol, $81 \%$ yield) as a colorless solid. ${ }^{1} \mathrm{H}$ NMR $\left(400 \mathrm{MHz},\left(\mathrm{CD}_{3}\right)_{2} \mathrm{SO}\right) \delta 8.02(\mathrm{dd}, J=7.7,1.4 \mathrm{~Hz}$, $1 \mathrm{H}, \operatorname{Ar} H), 7.97(\mathrm{~m}, 1 \mathrm{H}, \operatorname{Ar} H), 7.85(\mathrm{dd}, J=8.2,0.7 \mathrm{~Hz}, 1 \mathrm{H}, \operatorname{ArH}), 7.71(\mathrm{td}, J=7.6,1.2 \mathrm{~Hz}, 1$ $\mathrm{H}, \mathrm{ArH}) .{ }^{13} \mathrm{C}$ NMR $\left(100 \mathrm{MHz},\left(\mathrm{CD}_{3}\right)_{2} \mathrm{SO}\right) \delta 167.7,134.5,131.5,131.1,130.4,126.3,120.4$. IR v 3083 (w), 3060 (w), 2867 (w), 2402 (w), 1601 (m), 1585 (m), 1564 (m), 1440 (m), 1338 (s), 1302 (m), 1148 (m), $1018(\mathrm{w}), 834(\mathrm{~m}), 798(\mathrm{w}), 740(\mathrm{~s}), 694(\mathrm{~s}), 674(\mathrm{~m}), 649(\mathrm{~m}){ }^{1}$

\section{Trimethylsilyl(triisopropylsilyl)acetylene (8)}

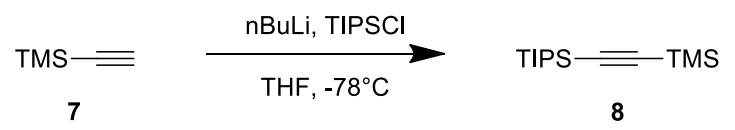

Following a modified reported procedure ${ }^{2}$, a 4-neck 500 mL flask equipped with a thermometer, a dropping funnel, a magnetic stirrer and a nitrogen inlet was charged with trimethylsilylacetylene (7) $(30.3 \mathrm{ml}, 213 \mathrm{mmol}, 1.0$ equiv.) under nitrogen . THF (330 mL) was added via a dropping funnel and the solution was cooled to $-78^{\circ} \mathrm{C}$. ${ }^{n} \mathrm{BuLi}(2.5 \mathrm{M}$ in hexanes, $86 \mathrm{~mL}, 0.21 \mathrm{~mol}, 0.98$ equiv) was added and the reaction mixture was stirred for 5 minutes at $-78^{\circ} \mathrm{C}$, then warmed to $0^{\circ} \mathrm{C}$ and stirred for 5 minutes. ${ }^{i} \mathrm{Pr}_{3} \mathrm{SiCl}(45.5 \mathrm{~mL}, 213 \mathrm{mmol}, 1$ equiv) was added dropwise via a dropping funnel at $-78^{\circ} \mathrm{C}$. The mixture was then allowed to warm to r.t. and stirred overnight. A saturated solution of $\mathrm{NH}_{4} \mathrm{Cl}_{\mathrm{aq}}(300 \mathrm{~mL})$ was added and the reaction was extracted with $\mathrm{Et}_{2} \mathrm{O}(2 \mathrm{x}$ $300 \mathrm{~mL}$ ). The organic layer was dried over $\mathrm{MgSO}_{4}$, filtered and concentrated. Distillation of the crude product $\left(1.4 \mathrm{mbar}, 55^{\circ} \mathrm{C}\right)$ afforded trimethylsilyl (triisopropylsilyl) acetylene $(\mathbf{8})(51.4 \mathrm{~g}$, $203 \mathrm{mmol}, 95 \%)$ as a colorless liquid. ${ }^{1} \mathrm{H}$ NMR (400 MHz, $\left.\mathrm{CDCl}_{3}\right) \delta 1.08$ (m, $\left.21 \mathrm{H}, \mathrm{TIPS}\right), 0.18$ (s, 9 H, TMS). IR v 2959 (m), 2944 (m), 2896 (w), 2867 (m), 1464 (w), 1385 (w), 1250 (m), 996 (w), $842(\mathrm{~s}), 764(\mathrm{~s}), 675(\mathrm{~m}), 660(\mathrm{~m}){ }^{2}$

${ }^{1}$ Kraszkiewicz, L.; Skulski, L. Arkivoc 2003, 6, 120.

${ }^{2}$ Helal, C J.; Magriotis, P. A.; Corey, E. J. J. Am. Chem. Soc. 1996, 118, 10938. 


\section{1-[(Triisopropyllsilyl)ethynyl]-1,2-benziodoxol-3(1H)-one (TIPS-EBX, (2))}

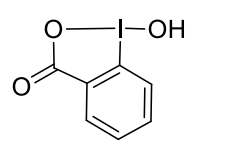

6

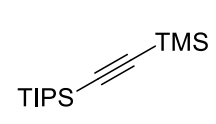

8

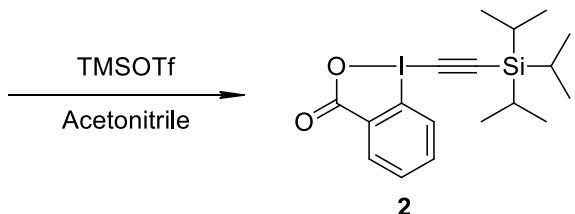

2

Caution: This reaction should be carried out behind a safety shield! ${ }^{3}$ Following a modified reported procedure, ${ }^{4}$ a 4 -neck flat-bottom flask equipped with a thermometer, a dropping funnel, a mechanic stirrer and a nitrogen inlet was charged with 2-iodosylbenzoic acid (6) (26.4 g, 100 mmol, 1.0 equiv). The system was flushed with $\mathrm{N}_{2}$ by three vacuum/ $\mathrm{N}_{2}$ cycles. Anhydrous acetonitrile $(350 \mathrm{~mL})$ was then canulated. The reaction mixture (white suspension) was cooled to $4^{\circ} \mathrm{C}$ and then trimethylsilyltriflate $(20.0 \mathrm{~mL}, 110 \mathrm{mmol}, 1.1$ equiv) was added dropwise for 15 min via a dropping funnel. The dropping funnel was rinsed with anhydrous acetonitrile $(10 \mathrm{~mL})$. No increase of temperature was observed. The ice bath was removed and the reaction stirred for 15 min. Trimethylsilyl(triisopropylsilyl)acetylene (8) $(28.0 \mathrm{~g}, 110 \mathrm{mmol}, 1.1$ equiv) was added dropwise via dropping funnel over $15 \mathrm{~min}$ (the colorless suspension was converted to a yellow solution). The dropping funnel was rinsed with anhydrous acetonitrile $(10 \mathrm{~mL})$ and the reaction was stirred for $30 \mathrm{~min}$. Then pyridine $(9.9 \mathrm{~mL}, 25 \mathrm{mmol}, 1.1$ equiv) was added dropwise via a dropping funnel over $5 \mathrm{~min}$. After $15 \mathrm{~min}$, the reaction mixture was transferred in a one-neck 1L flask and concentrated under reduced pressure until a solid was obtained. The solid was dissolved in $\mathrm{CH}_{2} \mathrm{Cl}_{2}(250 \mathrm{~mL})$ and transferred in a $2 \mathrm{~L}$ separatory funnel. The organic layer was added and washed with $1 \mathrm{M} \mathrm{HCl}(150 \mathrm{~mL})$ and the aqueous layer was extracted with $\mathrm{CH}_{2} \mathrm{Cl}_{2}(250 \mathrm{~mL})$. The organic layers were combined, washed with a saturated solution of $\mathrm{NaHCO}_{3}(2 \times 250 \mathrm{~mL})$, dried over $\mathrm{MgSO}_{4}$, filtered and the solvent was evaporated under reduced pressure. The resulting solid (44.8 g) was then recristallized in $\mathrm{CH}_{3} \mathrm{CN}(110 \mathrm{~mL})$. The colorless solid obtained over cooling down was then filtered over a Büchner funnel, washed with hexanes $(2 \times 40 \mathrm{~mL})$ and dried for $1 \mathrm{~h}$ at $40^{\circ} \mathrm{C}$ at 5 mbar. TIPS-EBX (2) $(36.2 \mathrm{~g}, 84.5 \mathrm{mmol}, 85 \%)$ was obtained as white crystals. Mp $173-177^{\circ} \mathrm{C}$ (decomposition). ${ }^{1} \mathrm{H}$ NMR $\left(400 \mathrm{MHz}, \mathrm{CDCl}_{3}\right) \delta 8.37(\mathrm{~m}, 1 \mathrm{H}, \mathrm{ArH}), 8.28(\mathrm{~m}, 1 \mathrm{H}$, $\mathrm{ArH}), 7.72(\mathrm{~m}, 2 \mathrm{H}, \mathrm{ArH}), 1.13(\mathrm{~m}, 21 \mathrm{H}, \mathrm{TIPS}) .{ }^{13} \mathrm{C}$ NMR $\left(101 \mathrm{MHz}, \mathrm{CDCl}_{3}\right) \delta 166.4,134.5$, $132.3,131.4,131.4,126.1,115.6,113.9,64.7,18.4,11.1 .^{4}$

\footnotetext{
${ }^{3}$ Differential scanning calorimetry showed that TIPS-EBX undergoes exothermic degradation at $187{ }^{\circ} \mathrm{C}$.

${ }^{4}$ Zhdankin, V. V.; Kuehl, C. J.; Krasutsky, A. P.; Bolz, J. T.; Simonsen, A. J. J. Org. Chem. 1996, $61,6547$.
} 


\section{Synthesis of tetrakis(acetonitrile)palladium(II)tetrafluoroborate}

Using a slight modification of a literature procedure,$^{5}$ a solution of palladium(II)chloride $(0.80 \mathrm{~g}$, $4.5 \mathrm{mmol}, 1.0$ equiv.) in anhydrous $\mathrm{MeCN}$ (40 mL, degassed by 3 "Freeze-Pump-Thaw" cycles) was prepared in a $250 \mathrm{~mL}$ 2-necked flask. Then $\mathrm{AgBF}_{4}(1.7 \mathrm{~g}, 9.0 \mathrm{mmol}, 2.0$ equiv.) was added as a solid. The flask was rinsed with anhydrous MeCN (24 mL). After $1.5 \mathrm{~h}$, the formed yellowish precipitate was filtered under nitrogen atmosphere. The filtrate was reduced to half of its original volume under vacuum. In order to precipitate the product, anhydrous $\mathrm{Et}_{2} \mathrm{O}(120 \mathrm{~mL})$ was canulated to the remaining solution. After filtration under nitrogen atmosphere and washing the solid with anhydrous $\mathrm{Et}_{2} \mathrm{O}(2 \times 20 \mathrm{~mL})$, the remaining solid was dried under vacuum overnight to afford $\mathrm{Pd}(\mathrm{MeCN})_{4}\left(\mathrm{BF}_{4}\right)_{2}(2.1 \mathrm{~g}, 4.5 \mathrm{mmol}$, quant.) as grey solid, which was used without further purification (no difference of activity was observed compared to a recrystallized product from MeCN from another synthesis). IR 3007 (w), 2948 (w), 2352 (w), 2321 (w), 1418 (w), $1370(\mathrm{w}), 1287$ (w), 1056 (s), 1024 (s), 964 (w), 769 (w), 623 (w). IR data corresponded to the literature values. ${ }^{6}$

\section{Synthesis of starting materials}

1-methyl-1H-indole (1a) and 6-bromo-1-methyl-1H-indole (1f) are commercially available.

\section{General procedure for methylation of indoles:}

The indole (1 equiv., 0.6 - $6.4 \mathrm{mmol}, 200$ - $1000 \mathrm{mg}$ ) was dissolved in dry THF in a 10 or $25 \mathrm{~mL}$ round-bottomed flask to give a $0.3 \mathrm{M}$ solution. Sodium hydride (60\% in mineral oil, 1.5 equiv.) was slowly added under a $\mathrm{N}_{2}$ flow at $0{ }^{\circ} \mathrm{C}$ to give a suspension. After stirring for $15 \mathrm{~min}$ at $0^{\circ} \mathrm{C}$ the reaction mixture was allowed to warm to r.t.. After $1.5 \mathrm{~h}$ it was cooled back to $0^{\circ} \mathrm{C}$ and methyl iodide (1.3 equiv.) was added. The mixture was then warmed up to r.t. and stirred overnight. After cooling back to $0^{\circ} \mathrm{C}$, it was quenched with water $(10 \mathrm{~mL})$, extracted with $\mathrm{Et}_{2} \mathrm{O}(3$ x $10 \mathrm{~mL}$ ), the organic layer was dried over $\mathrm{MgSO}_{4}$, filtered and then the solvent was evaporated under reduced pressure. The residue was purified via flash column chromatography (Hex:EtOAc 1:99-20:80), and recrystallized from hexane to give the N-methylated indole.

\section{5-Iodo-1-methyl-1H-indole (1b)}

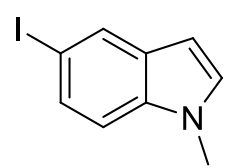

Starting from 5-iodo- $1 H$-indole $(1.00 \mathrm{~g}, 4.11 \mathrm{mmol})$, 5-iodo-1-methyl-1H-indole (1b) (0.768 g, $2.99 \mathrm{mmol}, 73 \%$ yield) was obtained as a white solid. $\mathrm{R}_{\mathrm{f}}: 0.65$ (hexanes:EtOAc 10:1). Mp: 76-78 ${ }^{\circ} \mathrm{C} .{ }^{1} \mathrm{H}$ NMR (400 $\left.\mathrm{MHz}, \mathrm{CDCl}_{3}\right) \delta 7.98(\mathrm{~s}, 1 \mathrm{H}$, ArH), 7.49 (d, $1 \mathrm{H}, J=8.6 \mathrm{~Hz}, \operatorname{ArH}), 7.13$ (d, $1 \mathrm{H}, J=8.6 \mathrm{~Hz}, \operatorname{ArH}), 7.04$ (s, $1 \mathrm{H}$, $\mathrm{ArH}), 6.43$ (s, $1 \mathrm{H}, \mathrm{ArH}), 3.80$ (s, $3 \mathrm{H}, \mathrm{Me}) .{ }^{13} \mathrm{C} \mathrm{NMR}\left(101 \mathrm{MHz}, \mathrm{CDCl}_{3}\right) \delta 135.8,131.0,129.8$, 129.7, 129.6, 111.3, 100.3, 82.9, 33.0. IR 3093 (w), 3053 (w), 2940 (w), 2919 (w), 2886 (w),

\footnotetext{
${ }^{5}$ Werner, H.; Bertleff, W.; Schubert, U., Inorg. Chim. Acta. 1980, 43, 199.

${ }^{6}$ Wayland, B. B.; Schramm, R. F. Inorg. Chem. 1969, 8, 971.
} 
2876 (w), 2856 (w), 1557 (m), 1510 (s), 1473 (s), 1432 (m), 1420 (s), 1379 (w), 1329 (m), 1277 (s), 1242 (s), 1193 (w), 1151 (w), 1103 (m), 1079 (m), 1045 (w), 1007 (m), 888 (s), 868 (m). HRMS (ESI) calcd for $\mathrm{C}_{9} \mathrm{H}_{9} \mathrm{IN}^{+}[\mathrm{M}+\mathrm{H}]^{+} 257.9774$; found 257.9776. NMR data is corresponding to the reported values. ${ }^{7}$

\section{5-Chloro-1-methyl-1H-indole (1c)}

Cl Starting from 5-chloro- $1 \mathrm{H}$-indole $(364 \mathrm{mg}, 2.40 \mathrm{mmol}$ ), 5-chloro-1-methyl- $1 \mathrm{H}$ indole (1c) $\left(268 \mathrm{mg}, 1.62 \mathrm{mmol}, 81 \%\right.$ yield) was obtained as a white solid. $\mathrm{R}_{\mathrm{f}}$ : 0.60 (hexanes:EtOAc 10:1). Mp: $33-34^{\circ} \mathrm{C}$, Lit $35^{\circ} \mathrm{C} .{ }^{1} \mathrm{H}$ NMR $(400 \mathrm{MHz}$, $\left.\mathrm{CDCl}_{3}\right) \delta 7.64(\mathrm{dd}, 1 \mathrm{H}, J=1.9,0.5 \mathrm{~Hz}, \mathrm{ArH}), 7.19-7.30(\mathrm{~m}, 2 \mathrm{H}), 7.10(\mathrm{~d}, 1 \mathrm{H}$, $J=3.1 \mathrm{~Hz}, \mathrm{ArH}), 6.47(\mathrm{dd}, 1 \mathrm{H}, J=3.1,0.7 \mathrm{~Hz}, \mathrm{ArH}), 3.80(\mathrm{~s}, 3 \mathrm{H}, \mathrm{Me}) \cdot{ }^{13} \mathrm{C}$ NMR $(101 \mathrm{MHz}$, $\left.\mathrm{CDCl}_{3}\right) \delta 135.1,130.1,130.1,125.1,121.8,120.2,110.2,100.6,33.1$. IR $3102(\mathrm{w}), 2943(\mathrm{w})$, 2913 (w), 2881 (w), 2817 (w), 1567 (w), 1513 (m), $1475(\mathrm{~s}), 1441$ (m), $1421(\mathrm{~s}), 1379$ (w), 1331 (m), 1278 (s), 1241 (s), 1199 (m), 1146 (m), 1106 (w), 1082 (m), 1063 (s), 1009 (m), 909 (m), 870 (m), 869 (m). HRMS (ESI) calcd for $\mathrm{C}_{9} \mathrm{ClH}_{9} \mathrm{~N}^{+}[\mathrm{M}+\mathrm{H}]^{+}$166.0418; found 166.0423. The NMR spectroscopic data is in accordance to those ones reported. ${ }^{8}$

\section{5-Fluoro-1-methyl-1H-indole (1d)}

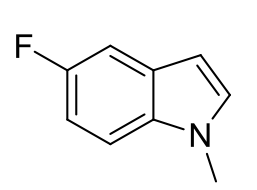

Starting from 5 -fluoro- $1 H$-indole $(541 \mathrm{mg}, 4.00 \mathrm{mmol})$, purification by column chromatography $\left(\mathrm{SiO}_{2}\right.$, hexane) gave pure 5-fluoro-1-methyl- $1 H$-indole (1d) $(536$ $\mathrm{mg}, 3.59 \mathrm{mmol}, 90 \%$ yield) as a white solid. $\mathrm{R}_{\mathrm{f}}$ : 0.70 (hexanes:EtOAc 10:1). Mp: 51-53 ${ }^{\circ} \mathrm{C} .{ }^{1} \mathrm{H}$ NMR $\left(400 \mathrm{MHz}, \mathrm{CDCl}_{3}\right) \delta 7.31(\mathrm{dd}, 1 \mathrm{H}, J=9.7,2.4 \mathrm{~Hz}, \mathrm{ArH})$, 7.26 (m, $1 \mathrm{H} \mathrm{ArH}), 7.12(\mathrm{~d}, 1 \mathrm{H}, J=3.1 \mathrm{~Hz}, \mathrm{ArH}), 7.01(1 \mathrm{H}, \mathrm{dt}, J=9.1,2 \mathrm{~Hz}, \mathrm{ArH}), 6.48$ (dd, 1 $\mathrm{H}, J=3.1,0.7 \mathrm{~Hz}, \mathrm{ArH}) .3 .81(\mathrm{~s}, 3 \mathrm{H}, \mathrm{Me}){ }^{13} \mathrm{C} \mathrm{NMR}\left(101 \mathrm{MHz}, \mathrm{CDCl}_{3}\right) \delta 158.0(\mathrm{~d}, J \mathrm{C}-\mathrm{F}=232$ $\mathrm{Hz}), 133.4,130.4,128.7(\mathrm{~d}, J \mathrm{C}-\mathrm{F}=10 \mathrm{~Hz}), 109.9(\mathrm{~d}, J \mathrm{C}-\mathrm{F}=15 \mathrm{~Hz}), 109.8,105.5(\mathrm{~d}, J \mathrm{C}-\mathrm{F}=$ $23 \mathrm{~Hz}$ ), 100.8 (d, $J$ C-F = 5 Hz), 33.1. IR 3104 (w), 2946 (w), 2922 (w), 2907 (w), 2887 (w), $2362(\mathrm{w}), 2343(\mathrm{w}), 1626(\mathrm{w}), 1576(\mathrm{w}), 1514(\mathrm{~m}), 1492$ (s), $1449(\mathrm{~m}), 1423(\mathrm{~m}), 1340(\mathrm{~m}), 1283$ (m), 1238 (s), 1228 (s), 1140 (m), 1129 (m), 1122 (m), 1100 (m), 1081 (m), 1013 (w), 949 (m), $859(\mathrm{~m}), 811$ (s). ${ }^{1} \mathrm{H}$ NMR is corresponding to the literature data. ${ }^{9}$

\section{5-Bromo-1-methyl-1H-indole (1e)}

Starting from 5-bromo- $1 H$-indole $(294 \mathrm{mg}, 1.50 \mathrm{mmol})$, 5-bromo-1-methyl$1 H$-indole $(\mathbf{1 e})(229 \mathrm{mg}, 1.10 \mathrm{mmol}, 73 \%)$ was obtained as an off-white solid. Rf: 0.45 (hexanes:EtOAc 10:1). Mp. $40-41{ }^{\circ} \mathrm{C}$, Lit.: $41{ }^{\circ} \mathrm{C} .{ }^{8}{ }^{1} \mathrm{H}$ NMR $(400 \mathrm{MHz}$, $\left.\mathrm{CDCl}_{3}\right) \delta 7.65(\mathrm{~d}, 1 \mathrm{H}, J=1.7 \mathrm{~Hz}, \mathrm{ArH}), 7.19(\mathrm{~m}, 1 \mathrm{H}, \mathrm{ArH}), 7.07(\mathrm{~d}, 1 \mathrm{H}, J=$ $8.7 \mathrm{~Hz}, \mathrm{ArH}), 6.93(\mathrm{~d}, 1 \mathrm{H}, J=3.1 \mathrm{~Hz}, \mathrm{ArH}), 6.32(\mathrm{dd}, 1 \mathrm{H}, J=3.1,0.8 \mathrm{~Hz}, \mathrm{ArH}), 3.64(\mathrm{~s}, 3 \mathrm{H}$, $\left.\mathrm{CH}_{3}\right) .{ }^{13} \mathrm{C}$ NMR $\left(101 \mathrm{MHz}, \mathrm{CDCl}_{3}\right) \delta 135.4,130.1,130.0,124.3,123.3,112.7,110.7,100.6$, 33.0. IR 3101 (w), 3065 (w), 2983 (w), 2947 (w), 2917 (w), 2869 (w), 2842 (w), $1606(w), 1557$ (m), $1517(\mathrm{w}), 1486(\mathrm{~m}), 1468(\mathrm{~m}), 1445(\mathrm{~m}), 1422(\mathrm{w}), 1421(\mathrm{w}), 1408(\mathrm{~m}), 1384(\mathrm{w}), 1353(\mathrm{w})$, $1334(\mathrm{~m}), 1320$ (s), 1302 (s), 1266 (w), 1216 (m), 1200 (w), 1139 (w), 1100 (s), 1087 (m), 1071 (w), 1052 (w), 1011 (w), 951 (w), 917 (m), 882 (w), 853 (w), 832 (w), 814 (m). HRMS (ESI)

\footnotetext{
${ }^{7}$ René, O.; Fagnou, K. Org. Lett. 2010, 12, 2116.

8 Klare, H. F.; Oestreich, M.; Ito, J.; Nishiyama, H.; Ohki, Y.; Tatsumi, K. J. Am. Chem. Soc. 2011, 133, 3312.5

9 Xu, X.-H.; Liu, G.-K.; Azuma, A.; Tokunaga, E.; Shibata, N. Org. Lett. 2011, 13, 4854.
} 
calcd for $\mathrm{C}_{9}{ }^{79} \mathrm{BrH}_{9} \mathrm{~N}^{+}[\mathrm{M}+\mathrm{H}]^{+}$209.9913; found 209.9901. The NMR corresponds to the reported data. $^{10}$

\section{7-Bromo-1-methyl-1H-indole (1g)}

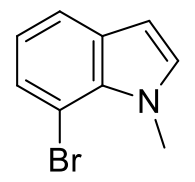

Starting from 7-bromo-1H-indole (392 mg, $2.00 \mathrm{mmol}$ ), 7-bromo-1-methyl- $\mathrm{H}$ indole (1g) (385mg, $1.833 \mathrm{mmol}, 92 \%$ yield) was obtained as white solid. $\mathrm{R}_{\mathrm{f}}: 0.80$ (hexanes:EtOAc 10:1). Mp 46-48 ${ }^{\circ} \mathrm{C}$, Lit $52^{\circ} \mathrm{C} .{ }^{10}{ }^{1} \mathrm{H} \mathrm{NMR}\left(400 \mathrm{MHz}, \mathrm{CDCl}_{3}\right) \delta 7.57$ $(\mathrm{dd}, J=7.8,1.0 \mathrm{~Hz}, 1 \mathrm{H}, \mathrm{ArH}), 7.37$ (dd, $1 \mathrm{H}, J=7.4,0.4 \mathrm{~Hz}, \mathrm{ArH}), 7.03$ (d, 1 $\mathrm{H}, J=3.1 \mathrm{~Hz}, \operatorname{ArH}), 6.95$ (t, $1 \mathrm{H}, J=7.7 \mathrm{~Hz}, \operatorname{ArH}), 6.49$ (d, $1 \mathrm{H}, J=3.1 \mathrm{~Hz}, \operatorname{ArH}), 4.19$ (s, $3 \mathrm{H}$, $\mathrm{Me}) .{ }^{13} \mathrm{C} \mathrm{NMR}\left(101 \mathrm{MHz}, \mathrm{CDCl}_{3}\right) \delta 133.1,131.8,131.7,126.6,120.5,120.4,103.9,101.2,36.9$. IR $3100(\mathrm{w}), 3066(\mathrm{w}), 3065(\mathrm{w}), 2947(\mathrm{w}), 2920(\mathrm{~m}), 2854(\mathrm{w}), 1557(\mathrm{~m}), 1517(\mathrm{w}), 1487(\mathrm{~m})$, 1467 (m), 1445 (m), 1408 (m), 1335 (m), 1320 (s), 1303 (s), 1216 (m), 1200 (w), 1101 (s), 1087 (w), $1052(\mathrm{w}), 917(\mathrm{~m}), 815$ (m). HRMS (ESI) calcd for $\mathrm{C}_{9}{ }^{79} \mathrm{BrH}_{9} \mathrm{~N}^{+}[\mathrm{M}+\mathrm{H}]^{+}$209.9913; found 209.9912. The NMR corresponds to the reported data. ${ }^{10}$

\section{1-Methyl-5-nitro-1H-indole (1h)}

$\mathrm{O}_{2} \mathrm{~N}$ Starting from 5-nitro- $1 \mathrm{H}$-indole (1.00 g, $\left.6.17 \mathrm{mmol}\right)$, 1-methyl-5-nitro-1Hindole (1h) (0.789 g, $4.48 \mathrm{mmol}, 73 \%$ yield) was obtained as yellow crystals. Rf: 0.20 (hexanes:EtOAc 10:1). Mp.: 170-172 ${ }^{\circ} \mathrm{C}$, Lit.: $169^{\circ} \mathrm{C} .{ }^{11}{ }^{1} \mathrm{H}$ NMR (400 $\left.\mathrm{MHz}_{\mathrm{CDCl}}\right) \delta 8.60(\mathrm{~d}, 1 \mathrm{H}, J=2.2 \mathrm{~Hz}, \mathrm{ArH}), 8.14(\mathrm{dd}, 1 \mathrm{H}, J=9.1,2.2 \mathrm{~Hz}, \mathrm{ArH}), 7.36(\mathrm{~d}, 1$ $\mathrm{H}, J=9.1 \mathrm{~Hz}, \mathrm{ArH}), 7.23(\mathrm{~d}, 1 \mathrm{H}, J=3.2 \mathrm{~Hz}, \mathrm{ArH}), 6.69$ (dd, $1 \mathrm{H}, J=3.2,0.8 \mathrm{~Hz}, \mathrm{ArH}), 3.88$ (s, $3 \mathrm{H}, \mathrm{Me}){ }^{13} \mathrm{C} \mathrm{NMR}\left(101 \mathrm{MHz}, \mathrm{CDCl}_{3}\right) \delta 142.9,135.3,134.6,133.3,120.7,114.8,106.4,102.2$, 33.3. IR 3104 (w), 2946 (w), 2936 (w), 2926 (w), 2919 (w), 2904 (w), $1614(w), 1580(w), 1519$ (s), 1481 (m), 1466 (m), 1465 (m), 1398 (w), 1333 (s), 1285 (m), 1243 (m), 1067 (m), 895 (w), 813 (w). HRMS (ESI) calcd for $\mathrm{C}_{9} \mathrm{H}_{9} \mathrm{~N}_{2} \mathrm{O}_{2}{ }^{+}[\mathrm{M}+\mathrm{H}]^{+}$177.0659; found 177.0662. The NMR corresponds to the literature data. ${ }^{12}$

\section{1-Methyl-6-nitro-1H-indole (1i)}

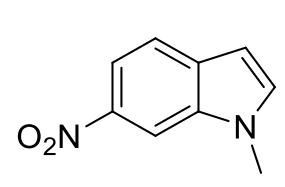

Starting from 6-nitro-1H-indole (649 mg, $4.00 \mathrm{mmol})$, 1-methyl-6-nitro-1Hindole (1i) (298 mg, $1.69 \mathrm{mmol}, 42 \%$ yield) was obtained as yellow needles. $\mathrm{R}_{\mathrm{f}}: 0.30$ (hexanes:EtOAc 10:1). Mp.: $78-80^{\circ} \mathrm{C}$. Lit: $77^{\circ} \mathrm{C} .{ }^{13}{ }^{1} \mathrm{H}$ NMR (400 $\left.\mathrm{MHz}_{\mathrm{CDCl}}\right) \delta 8.32(\mathrm{~d}, 1 \mathrm{H}, J=1.8 \mathrm{~Hz}), 8.02(\mathrm{dd}, 1 \mathrm{H}, J=8.8,2.0 \mathrm{~Hz}, \mathrm{ArH})$, $7.66(\mathrm{~d}, 1 \mathrm{H}, J=8.8 \mathrm{~Hz}, \mathrm{ArH}), 7.36(\mathrm{~d}, 1 \mathrm{H}, J=3.1 \mathrm{~Hz}, \mathrm{ArH}), 6.61(\mathrm{dd}, 1 \mathrm{H}, J=3.0,0.8 \mathrm{~Hz}$, ArH), 3.91 (s, $3 \mathrm{H}, \mathrm{Me}) .{ }^{13} \mathrm{C}$ NMR $\left(101 \mathrm{MHz} \mathrm{CDCl}_{3}\right) \delta 142.9,135.3,134.6,133.3,120.7,114.8$, 106.4, 102.2, 33.3. IR 3135 (w), 3122 (w), 3103 (w), 3079 (w), 2938 (w), 2909 (w), 2885 (w), 2813 (w), 1610 (w), 1584 (w), 1498 (s), 1463 (m), 1419 (m), 1408 (m), 1362 (m), 1335 (s), 1324 (s), 1300 (s), 1288 (s), 1235 (m), 1215 (w), 1134 (s), 1085 (w), 1063 (m), 933 (w), 880 (w), 843 (m), 817 (m). HRMS (ESI) calcd for $\mathrm{C}_{9} \mathrm{H}_{9} \mathrm{~N}_{2} \mathrm{O}_{2}{ }^{+}[\mathrm{M}+\mathrm{H}]^{+}$177.0659; found 177.0659. The NMR corresponds to the literature data. ${ }^{14}$

\footnotetext{
${ }^{10}$ Stadlwieser, J. F.; Dambaur, M. E. Helv. Chim. Acta 2006, 89, 936.

${ }^{11}$ Challis, B. C.; Lawson, A. J. J. Chem Soc., Perkin Trans. 2 1973, 918.

12 US patent, WO2009/42907, 2009.

${ }^{13}$ Coullet, F.; Morel, S.; Boyer, G.; Galy, J. P. Synth. Commun. 1998, $28,147$.

${ }^{14}$ Lane, B. S.; Brown, M. A.; Sames, D. J. Am. Chem. Soc. 2005, 127, 8050.
} 


\section{5-Methoxy-1-methyl-1H-indole (1j)}

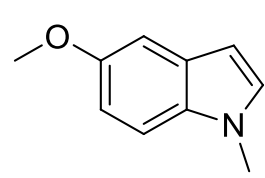

Starting from 5-methoxy- $1 H$-indole (221 mg, $1.50 \mathrm{mmol}) 5$-methoxy-1-methyl$1 H$-indole (104 mg, $0.645 \mathrm{mmol}, 43 \%$ yield) was obtained as white crystals. $\mathrm{R}_{\mathrm{f}}$ : 0.60 (hexanes:EtOAc 10:1). Mp.: 99-102 ${ }^{\circ} \mathrm{C}$, Lit.: $102-103^{\circ} \mathrm{C} .{ }^{15}{ }^{1} \mathrm{H}$ NMR $\left(400 \mathrm{MHz}, \mathrm{CDCl}_{3}\right) \delta 7.30(\mathrm{~d}, 1 \mathrm{H}, J=8.5 \mathrm{~Hz}, \mathrm{ArH}) 7.13(\mathrm{~s}, 1 \mathrm{H}, \mathrm{ArH}), 7.05(\mathrm{~s}$, $1 \mathrm{H}, \mathrm{ArH}), 6.92$ (d, $1 \mathrm{H}, J=8.8 \mathrm{~Hz}, \mathrm{ArH}), 6.43$ (d, $1 \mathrm{H}, J=1.0 \mathrm{~Hz}, \mathrm{ArH}), 3.90$ (s, $3 \mathrm{H}$ ), 3.80 (s, 3 $\mathrm{H}, \mathrm{Me}) .{ }^{13} \mathrm{C}$ NMR $\left(101 \mathrm{MHz}, \mathrm{CDCl}_{3}\right) \delta 154.0,132.2,129.3,128.8,111.9,109.9,102.5,100.4$, 55.9, 33.0. IR 2952 (w), 2918 (w), 2834 (w), 1622 (m), 1608 (w), 1577 (w), 1496 (s), 1459 (w), 1450 (m), 1449 (m), 1421 (s), 1347 (w), 1293 (w), 1243 (s), 1191 (m), 1152 (s), 1102 (w), 1026 (m), $942(\mathrm{w}), 855(\mathrm{~m}), 845(\mathrm{w}), 805$ (s). HRMS (ESI) calcd for $\mathrm{C}_{10} \mathrm{H}_{12} \mathrm{NO}^{+}[\mathrm{M}+\mathrm{H}]^{+}$162.0913; found 162.0914. The NMR corresponds to the spectral data from literature. ${ }^{16}$

\section{Other $\mathbf{N}$ substituted indoles}

\section{1-(3-phenylpropyl)-1H-indole (1k)}

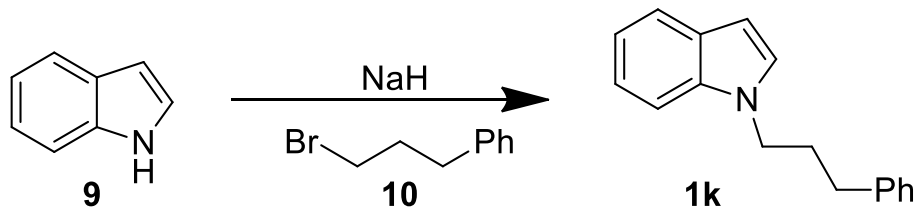

Following a reported procedure, ${ }^{17}$ in an oven-dried $10 \mathrm{~mL}$ round-bottomed flask $1 H$-indole (9) ( $0.644 \mathrm{~g}, 5.50 \mathrm{mmol}, 1.1$ equiv.) was dissolved in THF $(5 \mathrm{~mL})$ to give a colorless solution. Sodium hydride ( $60 \%$ in mineral oil, $0.240 \mathrm{~g}, 6.00 \mathrm{mmol}, 1.2$ equiv.) was added at $0^{\circ} \mathrm{C}$ and the reaction mixture was stirred for $30 \mathrm{~min}$. (3-bromopropyl) benzene (10) $(0.644 \mathrm{~mL}, 5.00 \mathrm{mmol}, 1$ eq.) was added dropwise. After 15 min the ice bath was removed and the reaction mixture was stirred for additional 4 hours, until there was no alkylating reagent (10) left according to TLC (Rf: 1.0, Hexanes:EtOAc 10:1) The reaction was cooled back to $0^{\circ} \mathrm{C}$, quenched with water, diluted with EtOAc $(10 \mathrm{~mL})$, extracted with water $(2 \times 10 \mathrm{~mL})$, washed with brine $(10 \mathrm{~mL})$ and dried over $\mathrm{MgSO}_{4}$. After filtration, the solvent was evaporated under reduced pressure. The crude product was purified by column chromatography $\left(\mathrm{SiO}_{2}\right.$, hexane:EtOAc $1 \%$ to $\left.10 \%\right)$ to give a colorless oil. This oil was then distillated (short path, Kugelrohr, $0.4 \mathrm{mbar}, 167-173^{\circ} \mathrm{C}$ ) to remove the 1,3-bis-alkylated indole. 1-(3-phenylpropyl)-1H-indole (1k) $(0.794 \mathrm{~g}, 3.37 \mathrm{mmol}, 68 \%$ yield) was obtained as a colorless oil. $\mathrm{R}_{\mathrm{f}}$ : 0.75 (hexanes:EtOAc 10:1). ${ }^{1} \mathrm{H}$ NMR $\left(400 \mathrm{MHz}, \mathrm{CDCl}_{3}\right) \delta$ $7.87(\mathrm{~d}, 1 \mathrm{H}, J=8 \mathrm{~Hz}, \mathrm{ArH}), 7.53-7.23(\mathrm{~m}, 9 \mathrm{H}, \mathrm{ArH}), 6.72(\mathrm{dd}, 1 \mathrm{H}, J=3.1,0.8 \mathrm{~Hz}, \mathrm{ArH}), 4.26$ (t, $2 \mathrm{H}, J=7.1 \mathrm{~Hz}, \mathrm{CH}_{2}$ ), 2.77 (t, $2 \mathrm{H}, J=8 \mathrm{~Hz}, \mathrm{CH}_{2}$ ), 2.34 (qi, $2 \mathrm{H}, J=7.8 \mathrm{~Hz},-\mathrm{CH}_{2}-\mathrm{CH}_{2}-\mathrm{CH}_{2}$ ). ${ }^{13} \mathrm{C}$ NMR $\left(101 \mathrm{MHz}, \mathrm{CDCl}_{3}\right) \delta 141.2,136.2,128.7,128.7,128.6,128.0,126.3,121.6,121.2$, 119.5, 109.6, 101.3, 45.8, 33.2, 31.7. IR 3085 (w), 3057 (w), 3026 (w), 3004 (w), 2946 (w), 2945 (w), $2870(\mathrm{w}), 1780(\mathrm{w}), 1738$ (s), 1717 (s), $1612(\mathrm{w}), 1603(\mathrm{w}), 1511(\mathrm{~m}), 1497(\mathrm{~m}), 1483(\mathrm{~m})$, 1464 (s), 1455 (s), 1400 (m), 1377 (s), 1354 (s), 1336 (s), 1315 (s), 1254 (s), 1207 (s), 1179 (m), 1166 (m), 1143 (m), 1143 (m), 1122 (m), 1114 (m), 1080 (m), 1031 (m), 1020 (m), 1004 (w), 952

\footnotetext{
${ }^{15}$ Flaugh, M. E.; Crowell, T. A.; Clemens, J. A.; Sawyer, B. D. J. Med. Chem. 1979, 22, 63.

${ }^{16}$ US patent, US2004/59131 A1, 2004.
} 
(w), 928 (w), 909 (w), 885 (m), 855 (w), 838 (w), 821 (w), $811($ w), 802 (w). HRMS (ESI) calcd for $\mathrm{C}_{17} \mathrm{H}_{18} \mathrm{~N}^{+}[\mathrm{M}+\mathrm{H}]^{+}$236.1434; found 236.1440. The NMR spectra correspond to the literature. ${ }^{17}$

\section{1-Benzyl-5-methoxy-1H-indole (11)}
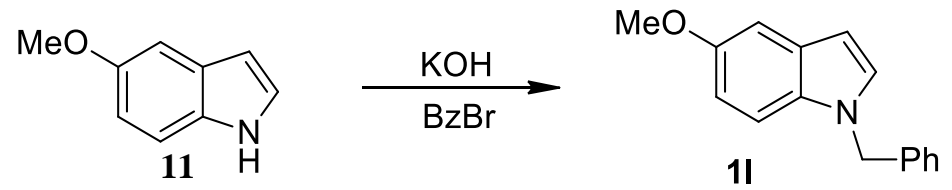

Following a reported method, ${ }^{18}$ 5-methoxy-1H-indole (11) $(300 \mathrm{mg}, 2.04 \mathrm{mmol}, 1$ equiv.) was dissolved in dry $\mathrm{EtOH}(17 \mathrm{~mL})$, to give a pale yellow solution. $\mathrm{KOH}$ (143 mg, $2.60 \mathrm{mmol}, 1.25$ equiv.) was added, and the reaction mixture was stirred until the base was dissolved. The solvent was evaporated under reduced pressure. The residue was dissolved in $17 \mathrm{~mL}$ acetone to give an orange solution. Then benzyl-bromide ( $244 \mu \mathrm{l}, 349 \mathrm{mg}, 2.04 \mathrm{mmol}, 1$ equiv.) was added, while a white solid precipitated from the mixture. The reaction was stirred for $15 \mathrm{~min}$, then the solid was filtered off, the liquid was concentrated, and purified by column chromatography $\left(\mathrm{SiO}_{2}, 5 \% \mathrm{Et}_{2} \mathrm{O}\right.$ in pentane), to give 1-benzyl-5-methoxy-1H-indole (1l) $(199 \mathrm{mg}, 0.839 \mathrm{mmol}, 41 \%)$ as a yellowish solid. $\mathrm{R}_{\mathrm{f}}$ : 0.33 (5\% $\mathrm{Et}_{2} \mathrm{O}$ in pentane). Mp.: 69-72 ${ }^{\circ} \mathrm{C}$ Lit: $66-68{ }^{\circ} \mathrm{C} .{ }^{19}{ }^{1} \mathrm{H}$ NMR (400

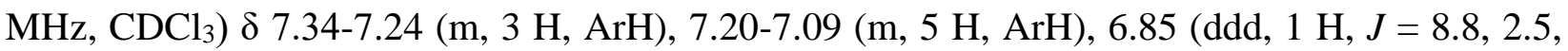
$0.3 \mathrm{~Hz}, \mathrm{ArH}), 6.49$ (dd, $1 \mathrm{H}, J=3.1,0.8 \mathrm{~Hz}, \mathrm{ArH}), 5.31\left(\mathrm{~s}, 2 \mathrm{H}, \mathrm{Bz} \mathrm{CH}_{2}\right), 3.86\left(\mathrm{~s}, 3 \mathrm{H}, \mathrm{OCH}_{3}\right)$. ${ }^{13} \mathrm{C}$ NMR $\left(101 \mathrm{MHz}, \mathrm{CDCl}_{3}\right) \delta 154.1,137.6,129.1,128.9,128.8,127.6,126.7,112.0,110.5$, 102.6, 101.2, 55.9, 50.3. ${ }^{20}$ IR 3063 (w), 3030 (w), 2995 (w), 2935 (w), 2917 (w), 2853 (w), 2853 (w), $2852(\mathrm{w}), 2831(\mathrm{w}), 1622(\mathrm{w}), 1576(\mathrm{w}), 1486(\mathrm{~s}), 1448(\mathrm{~m}), 1398(\mathrm{w}), 1356(\mathrm{w}), 1347(\mathrm{w})$, 1297 (w), 1255 (m), 1238 (s), 1192 (w), 1183 (w), 1149 (s), 1132 (m), 1030 (m), 836 (w), 797 (m), $752(\mathrm{~m}), 718(\mathrm{~s}), 717(\mathrm{~s}), 705(\mathrm{~s}), 704(\mathrm{~s}), 627(\mathrm{w})$. NMR is corresponding to the one in the literature. $^{19}$

\section{1-Benzyl-1H-indole (1m)}
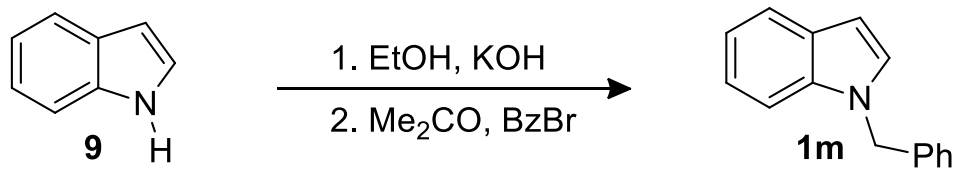

Following a reported method, ${ }^{18} 1 \mathrm{H}$-indole (9) (1.17 g, $10.0 \mathrm{mmol}, 1.00$ equiv.) was dissolved in ethanol (50 mL). $\mathrm{KOH} \mathrm{(566} \mathrm{mg,} 10.0 \mathrm{mmol}, 1.00$ equiv.) was added and the reaction mixture was stirred until the base dissolved. The solvent was evaporated under reduced pressure. The residue was dissolved in acetone (30 mL), and benzyl bromide was added dropwise, an exothermic reaction and precipitation was observed. After stirring for 30 minutes, the solid was filtered off. The liquid was concentrated under vacuum, and purified via column chromatography to give 1benzyl-1H-indole (1m) (586 mg, 28\% yield) as a white solid. $\mathrm{R}_{\mathrm{f}}: 0.75$ (hexanes:EtOAc 10:1) Mp.: 42-43 ${ }^{\circ} \mathrm{C}$, Lit: $41-43^{\circ} \mathrm{C},{ }^{21}{ }^{1} \mathrm{H}$ NMR $\left(400 \mathrm{MHz}, \mathrm{CDCl}_{3}\right) \delta 7.70(\mathrm{~d}, 1 \mathrm{H}, J=7.7 \mathrm{~Hz}, \mathrm{ArH})$,

\footnotetext{
${ }^{17}$ Jorapur, Y. R.; Jeong, J. M.; Chi, D. Y. Tetrahedron Lett. 2006, 47, 2435.

${ }^{18}$ Ottoni, O.; Cruz, R.; Alves, R. Tetrahedron 1998, 54, 13915.

${ }^{19}$ Evans, D. A.; Scheidt, K. A.; Fandrick, K. R.; Lam, H. W.; Wu, J. J. Am. Chem. Soc. 2003, 125, 10780.

${ }^{20}$ One Carbon signal was not resolved.

${ }^{21}$ Gribble, G. W.; Leiby, R. W.; Sheehan, M. N. Synthesis 1977, 856.
} 
7.41-7.09 (m, $9 \mathrm{H}, \mathrm{ArH}), 6.59$ (d, $1 \mathrm{H}, J=5 \mathrm{~Hz}, \mathrm{ArH}), 5.35$ (s, $\left.2 \mathrm{H}, \mathrm{Bz} \mathrm{CH}_{2}\right) .{ }^{13} \mathrm{C}$ NMR (101 $\left.\mathrm{MHz}, \mathrm{CDCl}_{3}\right) \delta 137.6,136.3,128.8,128.3,127.6,126.8,121.7,121.0,119.6,109.7,101.7,50.1$. IR 3099 (w), 3087 (w), $3086(w), 3056$ (w), 3030 (w), 3029 (w), $2919(w), 2858$ (w), $1613(w)$, 1612 (w), 1512 (s), 1496 (m), 1485 (m), 1464 (s), 1455 (s), 1439 (m), 1398 (m), 1357 (s), 1335 (s), 1318 (s), 1302 (s), 1256 (m), 1235 (w), 1208 (w), 1197 (m), 1182 (s), 1079 (w), 1062 (w), $1046(\mathrm{w}), 1030(\mathrm{~m}), 1012(\mathrm{~m}), 884(\mathrm{w}), 842(\mathrm{w}), 822(\mathrm{w})$. MS (ESI) calcd for $\mathrm{C}_{15} \mathrm{H}_{14} \mathrm{~N}^{+}[\mathrm{M}+\mathrm{H}]^{+}$ 208.1121; found 208.1122, The NMR spectral data is the same as in the literature. ${ }^{22}$

\section{1-Allyl-1H-indole (1n)}
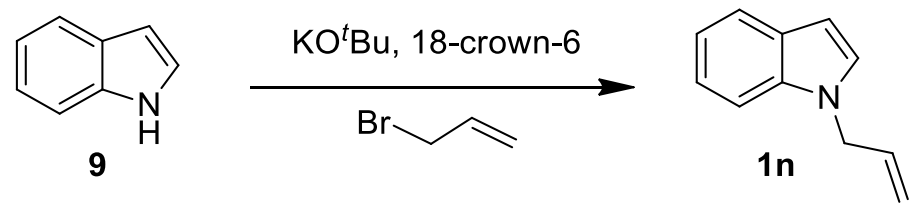

Following a reported procedure, ${ }^{23}$ potassium tertbutoxide (1234 mg, $11.00 \mathrm{mmol}, 1.1$ equiv.) was added to a solution of 18-crown-6 (26.4 mg, $0.100 \mathrm{mmol}, 0.01$ equiv.) in dry THF (25 mL). The mixture was stirred while $1 H$-indole (9) $(1.17 \mathrm{~g}, 10.0 \mathrm{mmol}, 1$ equiv.) was added in a single portion. The reaction was cooled to $0{ }^{\circ} \mathrm{C}$ in an ice bath. A solution of allyl bromide $(952 \mu 1,11.0$ mmol, 1.1 equiv.) in THF $(10 \mathrm{~mL})$ was added dropwise to the reaction mixture. After stirring for $4 \mathrm{~h}$, water $(20 \mathrm{~mL})$ was added to the reaction mixture, the layers were separated, and the aqueous layer was extracted with $\mathrm{Et}_{2} \mathrm{O}(2 \times 20 \mathrm{~mL})$. The combined organic layers were extracted with bringe $(50 \mathrm{~mL})$ and then dried over anhydrous $\mathrm{MgSO}_{4}$. The solvent was removed by evaporation under reduced pressure, and the residue was purified by column chromatography to give a mixture of 1-allylindole and 1,3-diallyl indole (1.24 g) as a colorless oil. The mixture was purified by short-path (Kugelrohr) distillation $\left(104-105{ }^{\circ} \mathrm{C}, 0.4 \mathrm{mbar}\right)$ to give 1-allyl-1H-indole (1n) (95\% pure, $266 \mathrm{mg}, 1.69 \mathrm{mmol}, 17 \%$ yield). $\mathrm{R}_{\mathrm{f}}$ : 0.60 (hexanes:EtOAc 10:1), co-spotting with impurity. ${ }^{1} \mathrm{H}$ NMR $\left(400 \mathrm{MHz}, \mathrm{CDCl}_{3}\right) \delta 7.75(\mathrm{~d}, 1 \mathrm{H}, J=6.7, \mathrm{ArH}), 7.42$ (d, $1 \mathrm{H}, J=8.2$ $\mathrm{Hz}, \mathrm{ArH}), 7.31(\mathrm{t}, 1 \mathrm{H}, J=7.0 \mathrm{~Hz}, \mathrm{ArH}), 7.22$ (t, $1 \mathrm{H}, J=6.5 \mathrm{~Hz}, \mathrm{ArH}), 7.18(\mathrm{~d}, 1 \mathrm{H}, J=3.3 \mathrm{~Hz}$, ArH), 6.63 (dd, $1 \mathrm{H}, J=3.2,0.8 \mathrm{~Hz})$ 6.13-6.02 (m, $1 \mathrm{H}$, Allyl H), 5.31-5.14 (m, $2 \mathrm{H}$, allyl $\mathrm{CH}_{2}$ ), $4.80\left(\mathrm{dt}, 2 \mathrm{H}, J=5.4, J=1.4\right.$, allyl $\left.\mathrm{CH}_{2}\right) .{ }^{13} \mathrm{C} \mathrm{NMR}\left(101 \mathrm{MHz}, \mathrm{CDCl}_{3}\right) \delta 136.2,133.6,128.8$, $127.9,121.6,121.1,119.5,117.3,109.7,101.5,48.9$. NMR is corresponding to the literature data. $^{24}$

\section{(2-Iodoethoxy)triisopropylsilane (13)}

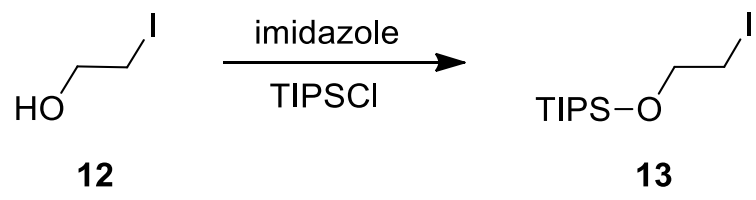

Following a reported procedure, ${ }^{25}$ 2-iodoethanol (12) $(1.10 \mathrm{~mL}, 10.0 \mathrm{mmol}, 1$ equiv.) was added to a solution of imidazole $(0.885 \mathrm{~g}, 13.0 \mathrm{mmol}, 1.3 \mathrm{equiv}$.) in DMF (5 mL) under an atmosphere of $\mathrm{N}_{2}$. Chlorotriisopropylsilane $(2.75 \mathrm{~mL}, 13.0 \mathrm{mmol}, 1.3$ equiv.) was added dropwise. After $1 \mathrm{~h}$ the reaction turned into a thick suspension, as a white solid precipitated. The ice bath was

\footnotetext{
${ }^{22}$ Kim, J.; Kim, H.; Chang, S. Org. Lett. 2012, 14, 3924.

${ }^{23}$ Guida, W. C.; Mathre, D. J. J. Org. Chem. 1980, 45, 3172.

${ }^{24}$ Choy, P. Y.; Lau, C. P.; Kwong, F. Y. J. Org. Chem. 2010, 76, 80.

${ }^{25}$ Bode, J. W.; Carreira, E. M. J. Org. Chem. 2001, 66, 6410.
} 
removed and the reaction mixture was stirred for an additional hour. Water $(5 \mathrm{~mL})$ was added to dissolve the solid. The organic layer was separated and washed through a $\mathrm{SiO}_{2}$ pad with pentane $(100 \mathrm{~mL})$. The solvent was evaporated, and the crude product was dried under vacuum to give (2iodoethoxy)triisopropylsilane (13) $\left(3.21 \mathrm{~g}, 9.78 \mathrm{mmol}, 98 \%\right.$ yield) as a colorless liquid. ${ }^{1} \mathrm{H}$ NMR (400 MHz, CDCl 3 ) $\delta 3.85\left(\mathrm{t}, J=6.9 \mathrm{~Hz}, 2 \mathrm{H}, \mathrm{CH}_{2}\right.$ ), 3.15 (t, $J=7.0 \mathrm{~Hz} .2 \mathrm{H}, \mathrm{CH}_{2}$ ), 1.11-0.88 (m, $21 \mathrm{H}$, TIPS). ${ }^{13} \mathrm{C} \mathrm{NMR}\left(101 \mathrm{MHz}, \mathrm{CDCl}_{3}\right) \delta$ 64.6, 18.0, 12.1, 6.9. IR $2958(\mathrm{~m}), 2942(\mathrm{~m}), 2891$ (w), 2866 (m), 1464 (m), 1384 (w), 1275 (w), 1249 (w), 1190 (w), 1169 (w), 1123 (s), 1092 (s), 1069 (s), 1013 (w), 999 (m), 943 (w), 920 (w), 882 (s), 857 (w). The NMR spectra is corresponding to the literature data. ${ }^{25}$

\section{1-(2-((Triisopropylsilyl)oxy)ethyl)-1H-indole (10)}
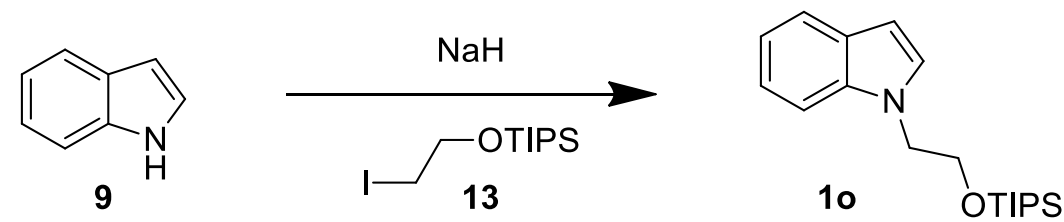

$1 H$-indole (9) (0.843 g, $7.20 \mathrm{mmol}, 1.2$ equiv.) was dissolved in N,N-dimethylformamide (6 mL) and $\mathrm{NaH}$ (60\% in mineral oil, $0.360 \mathrm{~g}, 9.00 \mathrm{mmol}, 1.33$ equiv., 1.25 equiv. compared to indole) was added at RT under strong stirring and the reaction mixture was stirred for one hour. N,NDimethylformamide $(18 \mathrm{~mL})$ was added to dissolve the white precipitae and to give a greenish solution. The reaction was cooled to $0{ }^{\circ} \mathrm{C}$ and (2-iodoethoxy)triisopropylsilane (13) (1.97 g, 6.00 mmol, 1equiv.) was added dropwise. The reaction was stirred overnight and let to slowly warm up to RT. The reaction was then quenched with water $(20 \mathrm{~mL})$ and the reaction mixture was extracted with EtOAc (3 x $25 \mathrm{~mL})$. The combined organic layers were washed with water (10 $\mathrm{mL})$, brine $(3 \times 10 \mathrm{~mL})$ and dried over $\mathrm{MgSO}_{4}$. The solvent was evaporated and the crude product was dried under vacuum with stirring. The crude NMR did not show the presence of the alkylating agent. TLC (10:1 hexanes: EtOAc, Rf prod.: 0.7). Purification by flash chromatography $\left(\mathrm{SiO}_{2}, 1 \%\right.$ to $10 \%$ EtOAc in hexane) gave 1-(2-((triisopropylsilyl)oxy)ethyl)$1 H$-indole (1o) (1.56 g, $4.91 \mathrm{mmol}, 82 \%$ yield) as a colorless oil, $\mathrm{R}_{\mathrm{f}}: 0.65$ (hexanes:EtOAc 10:1) ${ }^{1} \mathrm{H}$ NMR (400 MHz, $\left.\mathrm{CDCl}_{3}\right) \delta 7.66(\mathrm{~m}, 1 \mathrm{H}, \mathrm{ArH}), 7.38(\mathrm{dd}, 1 \mathrm{H}, J=8.2,0.8 \mathrm{~Hz}, \mathrm{ArH}), 7.25-$ $7.19(\mathrm{~m}, 2 \mathrm{H}, \mathrm{ArH}) 7.13(\mathrm{~m}, 1 \mathrm{H}, \mathrm{ArH}), 6.52(\mathrm{dd}, 1 \mathrm{H}, J=3.1,0.8 \mathrm{~Hz}, \mathrm{ArH}), 4.30(\mathrm{t}, 2 \mathrm{H}, J=6.0$ $\left.\mathrm{Hz}, \mathrm{CH}_{2}\right), 4.04\left(\mathrm{t}, 2 \mathrm{H}, J=5.8 \mathrm{~Hz}, \mathrm{CH}_{2}\right), 1.17-0.85$ (m, $\left.21 \mathrm{H}, \mathrm{TIPS}\right) .{ }^{13} \mathrm{C}$ NMR (101 MHz, $\left.\mathrm{CDCl}_{3}\right) \delta 136.1,128.7,128.6,121.3,120.9,119.2,109.3,101.0,62.8,48.8,17.9,11.9$. IR 3056 (w), 2942 (m), 2891 (m), 2865 (s), 1514 (w), 1464 (s), 1439 (w), 1400 (w), 1387 (w), 1360 (w), 1334 (w), 1317 (m), 1250 (w), 1200 (w), 1115 (s), 1077 (m), 1013 (m), 997 (w), 923 (m), 883 (s), 819 (w). HRMS (ESI) calcd for $\mathrm{C}_{19} \mathrm{H}_{32} \mathrm{NOSi}^{+}[\mathrm{M}+\mathrm{H}]^{+} 318.2248$; found 318.2236.

\section{1-(2-Bromoethyl)-1H-indole (1p)}
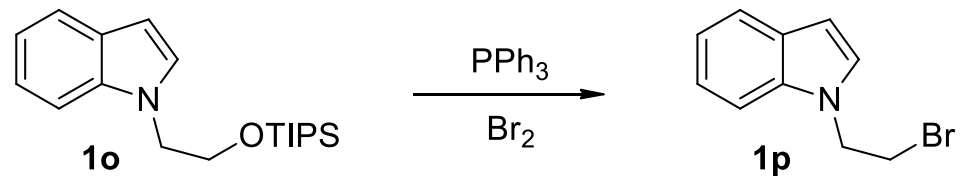
Following a modification of a reported procedure, ${ }^{26} \mathrm{Br}_{2}(0.170 \mathrm{~mL}, 3.30 \mathrm{mmol}, 1.1$ equiv. $)$ was added dropwise in 5 minutes to a solution of triphenylphosphine ( $866 \mathrm{mg}, 3.30 \mathrm{mmol}, 1.1$ equiv.) in DCM (3 mL) to give a slightly brownish suspension. The reaction mixture was stirred for 15 min, then 1-(2-((triisopropylsilyl)oxy)ethyl)-1H-indole (953 mg, $3.00 \mathrm{mmol}, 1$ equiv.) was added dropwise as a solution in DCM (3 mL). After $48 \mathrm{~h}$, the mixture became a pale yellow solution, and the TLC did not show any starting material. The reaction was quenched with water $(10 \mathrm{~mL})$, the layers were separated, and the organic layer was washed with water $(2 \mathrm{x} 10 \mathrm{~mL})$ and dried over $\mathrm{MgSO}_{4}$. After filtration, the solvent was removed under reduced pressure. Purification by column chromatography $\left(\mathrm{SiO}_{2}\right.$, hexane/EtOAc, $95 / 5$ to $\left.85 / 15\right)$ gave pure 1-(2-bromoethyl)-1Hindole (1p) (534 mg, $2.38 \mathrm{mmol}, 79 \%$ yield) as a colorless oil. Rf: 0.45 (hexanes : EtOAc 10:1). ${ }^{1} \mathrm{H}$ NMR (400 MHz, $\mathrm{CDCl}_{3}$ ) $\delta$ 7.73-7.63 (m, $\left.1 \mathrm{H}, \mathrm{ArH}\right), 7.41-7.25$ (m, $\left.2 \mathrm{H}, \mathrm{ArH}\right), 7.23-7.15$ (m, 2 $\mathrm{H}, \mathrm{ArH}), 6.58(\mathrm{dd}, 1 \mathrm{H}, J=3.2,0.8 \mathrm{~Hz}, \mathrm{ArH}), 4.59-4.49\left(\mathrm{~m}, 2 \mathrm{H}, \mathrm{CH}_{2}\right), 3.72-3.62\left(\mathrm{~m}, 2 \mathrm{H}, \mathrm{CH}_{2}\right)$. ${ }^{13} \mathrm{C}$ NMR $\left(101 \mathrm{MHz}, \mathrm{CDCl}_{3}\right) \delta 135.7,128.8,127.9,122.0,121.3,119.9,108.9,102.0,48.0,29.8$. IR $3101(w), 3100(w), 3086(w), 3053(w), 3027(w), 2962(w), 2961(w), 2917(w), 1612(w)$, 1514 (m), 1485 (m), 1477 (m), 1464 (s), 1453 (m), 1452 (m), 1436 (m), 1399 (m), 1355 (m), $1334(\mathrm{~m}), 1314$ (s), 1279 (m), 1241 (s), 1232 (m), 1218 (m), 1196 (m), 1166 (m), 1158 (m), 1119 (w), $1089(\mathrm{w}), 1040(\mathrm{w}), 1013(\mathrm{~m}), 927$ (w), 884 (m), 847 (w). HRMS (ESI) calcd for $\mathrm{C}_{10}{ }^{79} \mathrm{BrH}_{11} \mathrm{~N}^{+}[\mathrm{M}+\mathrm{H}]^{+} 224.0069$; found 224.0072. The obtained NMR data is in accordance with reported NMR values. ${ }^{26}$

\section{1-((1,3-Dioxolan-2-yl)methyl)-1H-indole (1q)}

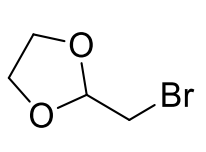

14

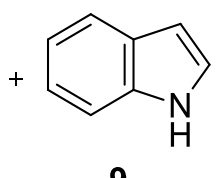

9

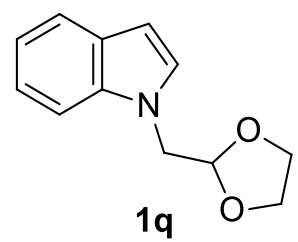

$1 \mathrm{q}$

$1 H$-indole (9) (586 mg, $5.00 \mathrm{mmol}, 1$ equiv) was dissolved in dry DMF (5 mL). The solution was then cooled to $0{ }^{\circ} \mathrm{C}$ and $\mathrm{NaH}(60 \%$ in mineral oil, $300 \mathrm{mg}, 7.50 \mathrm{mmol}, 1.5$ equiv) was added. The reaction mixture was stirred for $1 \mathrm{~h}$ at $\mathrm{RT}$ and a white solid precipitated. Additional DMF (15 $\mathrm{mL}$ ) was added, and 2-(bromomethyl)-1,3-dioxolane (14) (0.622 mL, $6.00 \mathrm{mmol}, 1.2$ equiv.) was added. The reaction mixture was stirred overnight at $50^{\circ} \mathrm{C}$, then cooled to RT, quenched with water and extracted with EtOAc $(3 \times 25 \mathrm{~mL})$. The combined organic layers were washed with water $(25 \mathrm{~mL})$, then brine $(2 \times 25 \mathrm{~mL})$, dried over $\mathrm{MgSO}_{4}$ and the solvent was evaporated to give a brownish crude oil. Purification by column chromatography $\left(\mathrm{SiO}_{2}\right.$, hexane/EtOAc 95/5 to 80/20) gave 1-((1,3-dioxolan-2-yl)methyl)- $1 H$-indole (1q) $(680 \mathrm{mg}, 3.35 \mathrm{mmol}, 67 \%$ yield) as colorless oil. $\mathrm{R}_{\mathrm{f}}$ : 0.2 (hexanes:EtOAc 10:1) ${ }^{1} \mathrm{H}$ NMR (400 MHz, $\left.\mathrm{CDCl}_{3}\right) \delta 7.68(\mathrm{~d}, 1 \mathrm{H}, J=7.9 \mathrm{~Hz}, \mathrm{ArH}$ ), $7.49(\mathrm{~d}, 1 \mathrm{H}, J=8.3 \mathrm{~Hz}, \mathrm{ArH}), 7.32-7.07$ (m, $3 \mathrm{H}, \mathrm{ArH}), 6.58(\mathrm{~d}, 1 \mathrm{H}, J=3.1 \mathrm{~Hz}, \mathrm{ArH}), 5.27$ (t, 1 $\left.\mathrm{H}, J=3.4 \mathrm{~Hz}, \mathrm{CH}_{2}-\mathrm{CH}\right), 4.37-4.24\left(\mathrm{~m}, 2 \mathrm{H}, \mathrm{N}-\mathrm{CH}_{2}\right), 3.81\left(\mathrm{~m}, 4 \mathrm{H}, \mathrm{CH}_{2}-\mathrm{CH}_{2}\right) .{ }^{13} \mathrm{C} \mathrm{NMR}(101$ $\left.\mathrm{MHz}_{2} \mathrm{CDCl}_{3}\right) \delta 136.8,129.0,128.5,121.6,120.8,119.5,109.8,102.5,101.7,65.3,49.2$. IR 3053 (w), 2973 (w), 2939 (w), 2887 (w), 2886 (w), 1683 (w), 1613 (w), 1514 (m), 1485 (m), 1475 (m), 1464 (s), 1398 (m), 1385 (w), 1366 (w), 1335 (m), 1316 (s), 1259 (m), 1258 (m), 1229 (w), 1228

${ }^{26}$ Bressy, C.; Alberico, D.; Lautens, M. J. Am. Chem. Soc. 2005, 127, 13148. 
(w), 1196 (m), 1142 (s), 1141 (s), 1093 (w), 1061 (m), 1036 (s), 1012 (s), 946 (m), 884 (w), 844

(m), 843 (m). HRMS (ESI) calcd for $\mathrm{C}_{12} \mathrm{H}_{14} \mathrm{NO}_{2}{ }^{+}[\mathrm{M}+\mathrm{H}]^{+}$204.1019; found 204.1025.

\section{Optimization of the alkynylation reaction}

\section{With isolated yields}

The optimization was carried out as following: TIPS EBX (2) was suspended/dissolved in the solvent, 1-methyl- $1 H$-indole (1a) $(25 \mu 1,26 \mathrm{mg}, 0.20 \mathrm{mmol}, 1$ equiv.) was added via a Hamilton syringe, then the additive was added, followed by the catalyst. The reaction was stirred overnight, the solvent was evaporated, the residue was taken up in EtOAc $(10 \mathrm{~mL})$, washed with $0.1 \mathrm{M}$ $\mathrm{NaOH}(10 \mathrm{~mL})$, saturated $\mathrm{NaHCO}_{3}(2 \times 10 \mathrm{~mL})$ and brine $(10 \mathrm{~mL})$, and dried over $\mathrm{MgSO}_{4}$. After filtration, the solvent was evaporated under reduced pressure, and purified via column chromatography.

\section{With GC yield}

The optimization was carried out as following: TIPS EBX (2) was suspended/dissolved in the solvent, 1-methyl-1H-indole (1a) $(25 \mu \mathrm{l}, 26 \mathrm{mg}, 0.20 \mathrm{mmol}, 1$ equiv.) was added via a Hamilton syringe, then the additive was added, followed by the catalyst. The reaction was stirred overnight. Then $10 \mu 1$ dodecanitrile was added via a Hamilton syringe. The mixture was homogenized then, ca. $100 \mu \mathrm{l}$ were transferred to a vial of $1 \mathrm{~mL}$ DCM. The GC yield was determined by the following calibration curve, using the ratio of the area of the product peak and the standard peak of the FID detector.

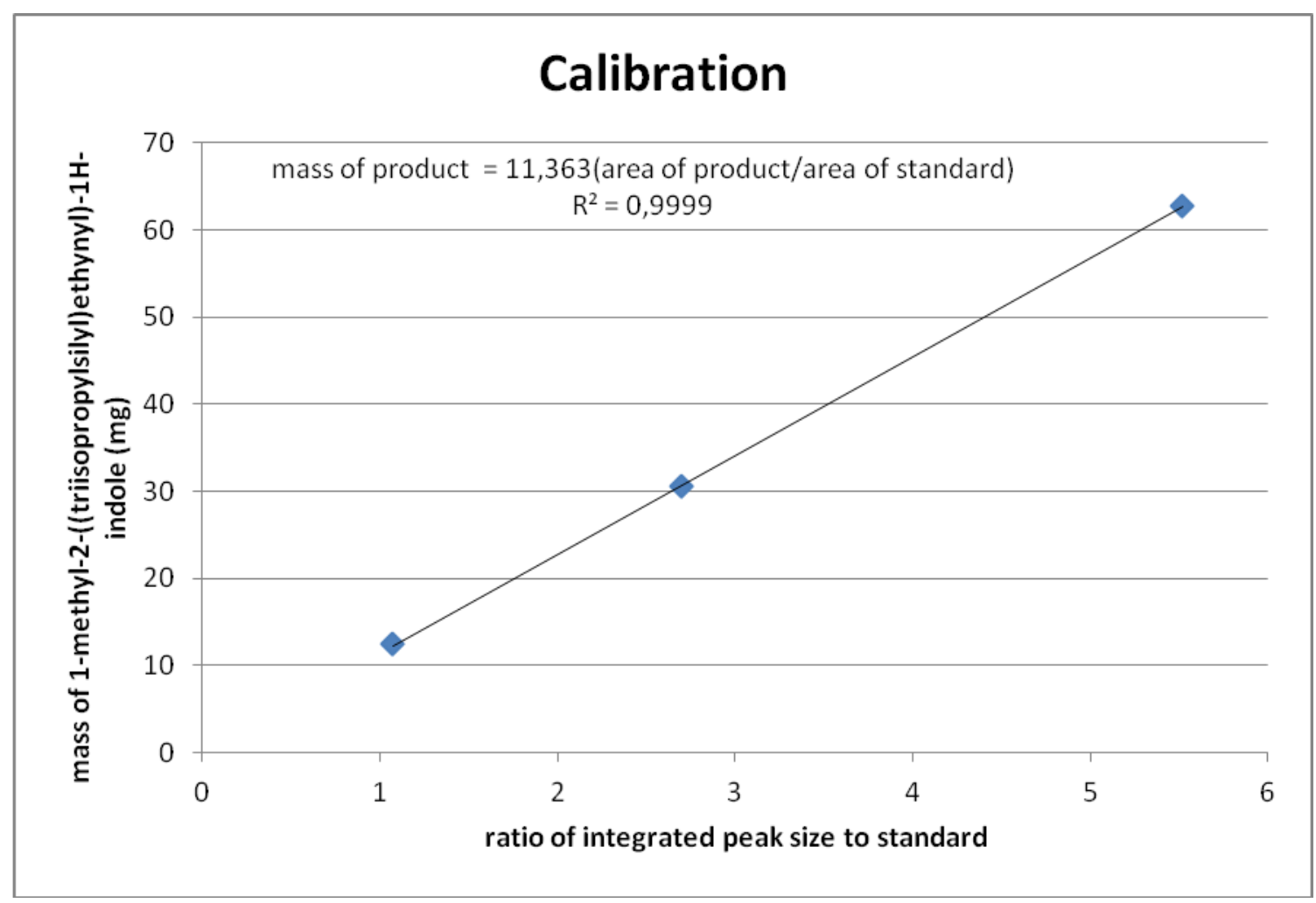




\section{Solvent effect}

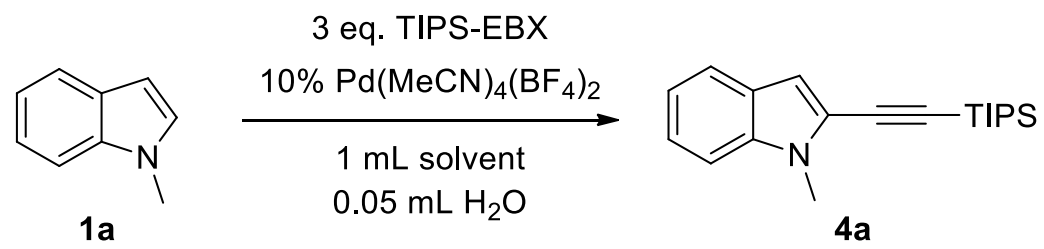

\begin{tabular}{|c|c|c|}
\hline Entry & Solvent & Isolated yield (\%) \\
\hline 1 & $\mathrm{Et}_{2} \mathrm{O}$ & 47 \\
\hline 2 & Toluene & 48 \\
\hline 3 & Acetonitrile & 16 \\
\hline 4 & Isopropanol & 32 \\
\hline 5 & Ethyl-acetate & 44 \\
\hline 6 & THF & 50 \\
\hline 7 & DMF & 37 \\
\hline 8 & DMSO & 0 \\
\hline 9 & EtOH & 6 \\
\hline 10 & $\mathrm{MeOH}$ & 10 \\
\hline
\end{tabular}

\section{TIPS-EBX dependence}

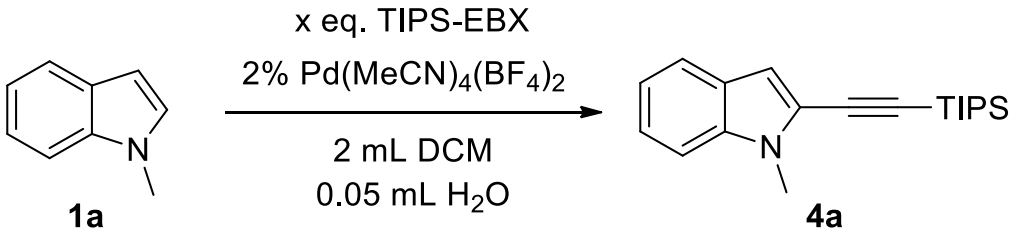

\begin{tabular}{|c|c|c|}
\hline Entry & Equivalent of TIPS EBX (2) & GC yield (\%) \\
\hline 1 & 0.5 & 16 \\
\hline 2 & 1 & 29 \\
\hline 3 & 2 & 51 \\
\hline 4 & 3 & 59 \\
\hline 5 & 5 & 57 \\
\hline
\end{tabular}




\section{Water dependence}

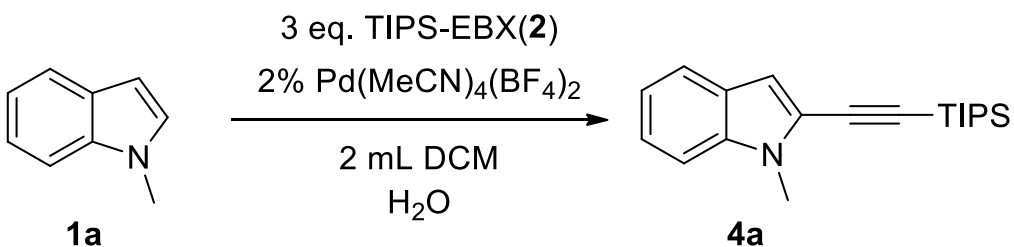

$1 \mathrm{a}$

\begin{tabular}{|c|c|c|}
\hline Entry & Water content $(\mu \mathrm{l})$ & GC yield $(\%)$ \\
\hline 1 & $0($ dry DCM $)$ & 22 \\
\hline 2 & 1.8 & 37 \\
\hline 3 & 100 & 61 \\
\hline 4 & 500 & 61 \\
\hline 5 & 1000 & 53 \\
\hline 6 & 2000 & 60 \\
\hline
\end{tabular}

\section{Selected additive screening}
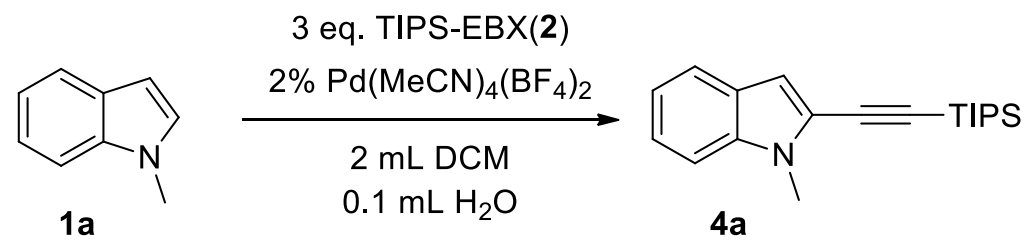

\begin{tabular}{|c|c|c|c|}
\hline Entry & Equivalent & Additive & GC yield (\%) \\
\hline 1 & - & - & 61 \\
\hline 2 & 1 & $\mathrm{Na}_{2} \mathrm{CO}_{3}$ & 16 \\
\hline 3 & 1 & $\mathrm{NaHCO}_{3}$ & 17 \\
\hline 4 & 1 & PivOH & 56 \\
\hline 5 & 10 & PivOH & 14 \\
\hline 6 & 5 & TFA & Traces \\
\hline 7 & 1 & $\mathrm{Cs}_{2} \mathrm{CO}_{3}$ & Traces \\
\hline 8 & 1 & $\mathrm{CsOPiv}$ & Traces \\
\hline 9 & 0.1 & TMEDA & 0 \\
\hline 10 & 0.1 & DMEDA & 0 \\
\hline 11 & 1 & Ethylene glycol & 8 \\
\hline
\end{tabular}




\section{Catalyst screening}

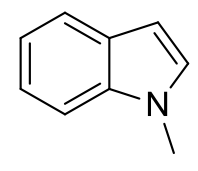

$1 \mathrm{a}$
Pd cat.

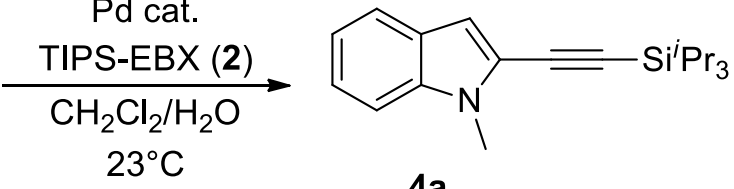

$4 a$

\begin{tabular}{|c|c|c|c|c|}
\hline Entry & loading & Pd source & ligand & GC yield (\%) \\
\hline 1 & $2 \%$ & $\mathrm{Pd}(\mathrm{MeCN})_{4}\left(\mathrm{BF}_{4}\right)_{2}$ & - & 61 \\
\hline 2 & $2 \%$ & $\mathrm{PdCl}_{2}$ & - & 22 \\
\hline 3 & $2 \%$ & $\mathrm{Pd}_{2} \mathrm{dba}_{3}$ & - & 57 \\
\hline 4 & $2 \%$ & $\mathrm{Pd}($ allyl $) \mathrm{codBF}_{4}$ & - & 44 \\
\hline 5 & $2 \%$ & $\mathrm{Pd}($ allyl $) \mathrm{Cl} \mathrm{dimer}$ & - & 60 \\
\hline 6 & $2 \%$ & $\mathrm{Pd}\left(\mathrm{PPh}_{3}\right)_{4}$ & - & traces \\
\hline 7 & $2 \%$ & $\mathrm{Pd}(\mathrm{dba})_{2}$ & - & 44 \\
\hline 8 & $2 \%$ & $\mathrm{Pd}_{2}(\mathrm{dba})_{3} \mathrm{CHCl}_{3}$ & - & 54 \\
\hline 9 & $2 \%$ & $\mathrm{Pd}_{2}(\mathrm{dba})_{3}$ & $\mathrm{dppp}$ & 24 \\
\hline 10 & $2 \%$ & $\mathrm{Pd}_{2}(\mathrm{dba})_{3}$ & $\mathrm{Xphos}$ & 26 \\
\hline 11 & $10 \%$ & $\mathrm{Pd}_{3}(\mathrm{TFA})_{2}$ & - & 42 \\
\hline 12 & $10 \%$ & $\mathrm{Pd}_{2}\left(\mathrm{MeCN}_{4}\left(\mathrm{BF}_{4}\right)_{2}\right.$ & - & 50 \\
\hline
\end{tabular}

\section{General procedure for $\mathrm{C} 2$ selective alkynylation of indoles.}<smiles>[R]c1cccc2cc[nH]c12</smiles>

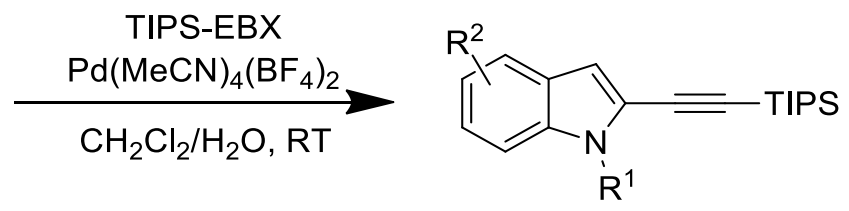

In a $10 \mathrm{~mL}$ round bottom-flask, the corresponding indole $(0.500 \mathrm{mmol}, 1.0$ equiv. $)$ and 1 [(triisopropylsilyl)ethynyl]-1,2-benziodoxol-3(1H)-one (TIPS-EBX, 2) $(643 \mathrm{mg}, 1.50 \mathrm{mmol}, 3$ equiv.) were dissolved in DCM $(5 \mathrm{~mL})$ under air, then water was added $(0.10 \mathrm{~mL})$. Lastly $\mathrm{Pd}(\mathrm{MeCN})_{4}\left(\mathrm{BF}_{4}\right)_{2}(4.4 \mathrm{mg}, 10 \mu \mathrm{mol}, 2 \%)$ was added with strong stirring. The flask was closed and the reaction mixture was stirred overnight (the reaction is generally completed after 4-6 h), when it became brownish. The solvent was evaporated under reduced pressure. EtOAc $(25 \mathrm{~mL})$ was added to the crude product, and the solution was washed with $\mathrm{NaOH}_{\mathrm{aq}}(0.1 \mathrm{M}, 25 \mathrm{~mL})$, conc. $\mathrm{NaHCO}_{3}(2 \times 25 \mathrm{~mL})$ and brine $(25 \mathrm{~mL})$. The organic layer was dried over $\mathrm{MgSO}_{4}$, filtered and 
the solvent was evaporated under reduced pressure. Purification by column chromatography gave the pure alkynylated product. ${ }^{27}$

\section{1-Methyl-2-((triisopropylsilyl)ethynyl)-1H-indole (4a)}

Starting from 1-methyl-1H-indole (1a) $(64 \mu \mathrm{l}, 66 \mathrm{mg}, 0.50 \mathrm{mmol}), 1$ -<smiles>Cn1c(C#C[In])cc2ccccc21</smilesmethyl-2-((triisopropylsilyl)ethynyl)-1H-indole (4a) (102 mg, 0.33 mmol, 66\%) was obtained as a pale yellow oil after purification by column chromatography $\left(\mathrm{SiO}_{2}\right.$, hexane to hexane/DCM 90/10), $\mathrm{R}_{\mathrm{f}}: 0.75$ (hexanes:EtOAc 10:1). ${ }^{1} \mathrm{H} \mathrm{NMR}\left(400 \mathrm{MHz}, \mathrm{CDCl}_{3}\right) \delta 7.63$ (dt, $\left.1 \mathrm{H}, J=8.0,0.9 \mathrm{~Hz}, \mathrm{ArH}\right), 7.33$ 7.28 (m, 2 H, ArH), 7.16 (q, 1 H, J = 4 Hz, ArH), 6.86 (s, $1 \mathrm{H}, \operatorname{ArH}), 3.78$ (s, $3 \mathrm{H}, \mathrm{Me}$ ), 1.30$1.09\left(\mathrm{~m}, 21 \mathrm{H}\right.$, TIPS) ${ }^{13} \mathrm{C}$ NMR $\left(101 \mathrm{MHz}, \mathrm{CDCl}_{3}\right) \delta 137.1,127.1,123.1,122.3,121.1,120.1$, 109.4, 107.7, 98.2, 97.8, 30.6, 18.8, 11.4. IR 3058 (w), 2942 (s), 2891 (m), 2864 (s), 2150 (s), 1463 (s), 1429 (w), 1383 (m), 1364 (m), 1339 (s), 1317 (m), 1238 (m), 1170 (w), 1152 (w), 1073 (w), 1012 (m), 997 (m), 920 (m), 883 (s), 854 (m). HRMS (ESI) calcd. for $\mathrm{C}_{20} \mathrm{H}_{30} \mathrm{NSi}^{+}[\mathrm{M}+\mathrm{H}]^{+}$ 312.2142; found 312.2147.

\section{5-Iodo-1-methyl-2-((triisopropylsilyl)ethynyl)-1H-indole (4b)}

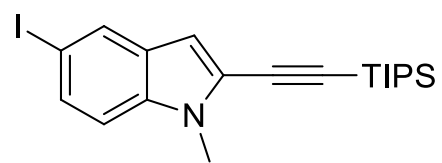

Starting from 5-iodo-1-methyl-1H-indole (1b) (129 mg, 0.500

mmol), 5-iodo-1-methyl-2-((triisopropylsilyl)ethynyl)-1H-indole (4b) (148 mg, $0.339 \mathrm{mmol}, 68 \%$ yield) was obtained as a white solid after purification by column chromatography $\left(\mathrm{SiO}_{2}\right.$, hexane to hexane/DCM 90/10). Rf: 0.85 (hexanes:EtOAc 10:1). Mp.: 76-78 ${ }^{\circ} \mathrm{C} .{ }^{1} \mathrm{H}$ NMR $\left(400 \mathrm{MHz}, \mathrm{CDCl}_{3}\right)$ $\delta 7.71(\mathrm{~d}, 1 \mathrm{H}, J=1.2 \mathrm{~Hz}, \mathrm{ArH}), 7.29(\mathrm{dd}, 1 \mathrm{H}, J=8.6,1.5 \mathrm{~Hz}, \mathrm{ArH}), 6.84$ (d, $1 \mathrm{H}, J=8.6 \mathrm{~Hz}$, ArH), 6.51 (s, $1 \mathrm{H}, \mathrm{ArH}), 3.60$ (s, $3 \mathrm{H}, \mathrm{Me}), 1.15-0.88$ (m, $21 \mathrm{H}, \mathrm{TIPS}) .{ }^{13} \mathrm{C}$ NMR (101 MHz, $\left.\mathrm{CDCl}_{3}\right) \delta 136.1,131.3,129.6,129.5,123.1,111.4,106.7,98.7,97.4,83.6,30.7,18.7,11.3$. IR 2957 (s), 2942 (s), 2891 (m), 2890 (m), 2865 (s), 2154 (m), 1557 (w), 1516 (w), 1465 (s), 1426 (w), 1425 (w), 1383 (m), 1367 (w), 1326 (m), 1274 (w), 1236 (w), 1169 (w), 1147 (w), 1101 (w), 1075 (w), 1063 (w), 1044 (w), 1018 (w), 997 (m), 911 (m), 883 (s). HRMS (ESI) calcd for $\mathrm{C}_{20} \mathrm{H}_{29} \mathrm{INSi}^{+}[\mathrm{M}+\mathrm{H}]^{+}$438.1109; found 438.1113.

\section{5-Chloro-1-methyl-2-((triisopropylsilyl)ethynyl)-1H-indole (4c)}

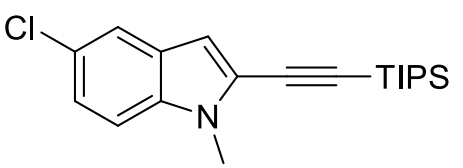

Starting from 5-chloro-1-methyl-1H-indole $(83 \mathrm{mg}, 0.50 \mathrm{mmol} \mathrm{5-}$ chloro-1-methyl-2-((triisopropylsilyl)ethynyl)-1H-indole (4c) (94.2 $\mathrm{mg}, 0.272 \mathrm{mmol}, 54 \%$ yield) was obtained as a white solid after purification by column chromatography $\left(\mathrm{SiO}_{2}\right.$, hexane to hexane/DCM 90/10). Mp.: 36-38 ${ }^{\circ} \mathrm{C} .{ }^{1} \mathrm{H}$ NMR (400 MHz, $\left.\mathrm{CDCl}_{3}\right) \delta 7.34$ (d, $1 \mathrm{H}, J=1.4 \mathrm{~Hz}$, ArH), 7.03-6.95 (m, 2 H, ArH), 6.53 (s, 1 H, ArH), 3.61 (s, 3 H, Me), 1.11-0.87 (m, 21 H, TIPS). ${ }^{13} \mathrm{C}$ NMR $\left(101 \mathrm{MHz}, \mathrm{CDCl}_{3}\right) \delta 135.5,127.9,125.8,123.7,123.4,120.2,110.4,107.1,98.7,97.5$, 30.8, 18.7, 11.3. IR 2943 (s), 2892 (w), 2865 (s), 2362 (w), 2341 (w), 2331 (w), 2153 (m), 1467 (s), $1427(\mathrm{w}), 1384(\mathrm{~m}), 1330(\mathrm{~m}), 1272(\mathrm{w}), 1235(\mathrm{w}), 1169(\mathrm{w}), 1160(\mathrm{w}), 1146(\mathrm{w}), 1101(\mathrm{w})$,

\footnotetext{
${ }^{27}$ No dry glassware is needed. The starting materials are giving a brownish-red color on anisaldehyde stain, while the less polar products are purple. The reaction is never observed to go until full conversion. The order of addition is crucial in term of yield.
} 
1064 (m), 1017 (w), 997 (w), 923 (m), 883 (s), 866 (w). HRMS (ESI) calcd for $\mathrm{C}_{20} \mathrm{ClH}_{29} \mathrm{NSi}^{+}$ $[\mathrm{M}+\mathrm{H}]^{+}$346.1752; found 346.1753.

\section{5-Fluoro-1-methyl-2-((triisopropylsilyl)ethynyl)-1H-indole (4d)}

Starting from 5-fluoro-1-methyl-1 $H$-indole (1d) $(74.6 \mathrm{mg}, 0.500$<smiles>Cn1c(C#C[In])cc2cc(F)ccc21</smiles>
mmol), 5-fluoro-1-methyl-2-((triisopropylsilyl)ethynyl)-1H-indole (4d) (74 mg, $0.23 \mathrm{mmol}, 45 \%$ yield) was obtained as a yellow oil after purification by column chromatography $\left(\mathrm{SiO}_{2}\right.$, hexane). $\mathrm{R}_{\mathrm{f}}$ : 0.30 (Hexanes) 0.9 (hexanes/EtOAc 10:1), ${ }^{1} \mathrm{H}$ NMR $\left(400 \mathrm{MHz}, \mathrm{CDCl}_{3}\right) \delta$ 7.03-6.95 (m, $2 \mathrm{H}$, ArH), $6.81(\mathrm{td}, 1 \mathrm{H}, J=9.1,2.5 \mathrm{~Hz}, \mathrm{ArH}), 6.55$ (d, $1 \mathrm{H}, J=0.7 \mathrm{~Hz}, \mathrm{ArH}), 3.61$ (s, 3H, Me), 1.16$0.87(\mathrm{~m}, 21 \mathrm{H}, \mathrm{TIPS}) .{ }^{13} \mathrm{C} \mathrm{NMR}\left(101 \mathrm{MHz}, \mathrm{CDCl}_{3}\right) \delta 158.3(\mathrm{~d}, J \mathrm{C}-\mathrm{F}=233 \mathrm{~Hz}), 133.8,127.1(\mathrm{~d}$, $J \mathrm{C}-\mathrm{F}=10 \mathrm{~Hz}), 123.8,111.7(\mathrm{~d}, J \mathrm{C}-\mathrm{F}=26 \mathrm{~Hz}), 110.1(\mathrm{~d}, J \mathrm{C}-\mathrm{F}=9.4 \mathrm{~Hz}), 107.4(\mathrm{~d}, J \mathrm{C}-\mathrm{F}=5.8$ Hz), 105.5(d, J C-F = 23 Hz), 98.4, 97.7, 30.8, 18.7, 11.3. IR 2943 (m), $2891(\mathrm{w}), 2865$ (m), 2151 (m), 1624 (w), 1580 (w), 1477 (s), 1430 (w), 1389 (m), 1366 (w), 1342 (m), 1282 (m), 1232 (w), 1191 (s), 1130 (w), 1116 (m), $1101(\mathrm{w}), 1073$ (w), 1017 (w), 997 (m), 955 (m), $921(\mathrm{w}), 883(\mathrm{~s})$, 853 (m). HRMS (ESI) calcd for $\mathrm{C}_{20} \mathrm{FH}_{29} \mathrm{NSi}^{+}[\mathrm{M}+\mathrm{H}]^{+} 330.2048$; found 330.2039.

\section{5-Bromo-1-methyl-2-((triisopropylsilyl)ethynyl)-1H-indole (4e)}

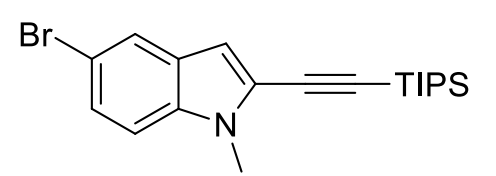

Starting from 5-bromo-1-methyl-1H-indole (1e) (105 mg, 0.500 mmol), 5-bromo-1-methyl-2-((triisopropylsilyl)ethynyl)-1H-indole (4e) (105 mg, $0.269 \mathrm{mmol}, 54 \%$ yield) as a white solid after purification by column chromatography $\left(\mathrm{SiO}_{2}\right.$, hexane). $\mathrm{R}_{\mathrm{f}}: 0.70$ (hexanes:EtOAc 10:1) Mp.: 59-60 ${ }^{\circ} \mathrm{C},{ }^{1} \mathrm{H}$ NMR (400 MHz, $\left.\mathrm{CDCl}_{3}\right) \delta 7.50$ (s, $\left.1 \mathrm{H}, \mathrm{ArH}\right), 7.13$ (d, $1 \mathrm{H}, J=8.7 \mathrm{~Hz}, \mathrm{ArH}), 6.93$ (d, $1 \mathrm{H}, J=8.7 \mathrm{~Hz}, \mathrm{ArH}), 6.52$ (s, $1 \mathrm{H}, \mathrm{ArH}), 3.60$ (s, $3 \mathrm{H}, \mathrm{Me}$ ), 1.05$0.89\left(\mathrm{~m}, 21 \mathrm{H}\right.$, TIPS). ${ }^{13} \mathrm{C}$ NMR $\left(101 \mathrm{MHz}, \mathrm{CDCl}_{3}\right) \delta 135.7,128.6,125.9,123.5,123.3,113.4$, 110.8, 106.9, 98.7, 97.4, 30.8, 18.7, 11.3. IR 2943 (s), 2890 (w), 2865 (s), 2362 (m), 2341 (w), 2335 (w), 2153 (m), 1517 (w), 1494 (m), 1466 (s), 1426 (w), $1386(\mathrm{w}), 1366$ (w), 1330 (m), 1283 (w), $1272(\mathrm{w}), 1238(\mathrm{~m}), 1191(\mathrm{w}), 1170(\mathrm{w}), 1141(\mathrm{w}), 1131(\mathrm{w}), 1124(\mathrm{w}), 1101(\mathrm{w}), 1074(\mathrm{w})$, 1052 (w), 1014 (w), 998 (w), $951(\mathrm{w}), 916$ (m), 884 (s), 866 (w), $857(\mathrm{w}), 837(\mathrm{w}), 826(\mathrm{w}), 812$ (w). HRMS (ESI) calcd for $\mathrm{C}_{20}{ }^{79} \mathrm{BrH}_{29} \mathrm{NSi}^{+}[\mathrm{M}+\mathrm{H}]^{+} 390.1247$; found 390.1252.

\section{6-Bromo-1-methyl-2-((triisopropylsilyl)ethynyl)-1H-indole (4f)}

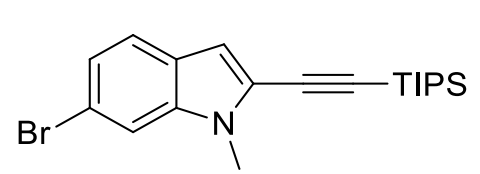

Starting from 6-bromo-1-methyl-1H-indole (1f) (105 mg, 0.500 mmol), 6-bromo-1-methyl-2-((triisopropylsilyl)ethynyl)-1 $H$-indole (4f) (140 mg, $0.359 \mathrm{mmol}, 72 \%$ yield) was obtained as a brown oil after purification by column chromatography $\left(\mathrm{SiO}_{2}\right.$, hexane), $\mathrm{R}_{\mathrm{f}}$ : 0.80 (hexanes:EtOAc 10:1) ${ }^{1} \mathrm{H}$ NMR $\left(400 \mathrm{MHz} \mathrm{CDCl}_{3}\right) \delta 7.22(\mathrm{~m}, 2 \mathrm{H}, \mathrm{ArH}), 7.01(\mathrm{~d}, 1 \mathrm{H}, J=$ $8.4 \mathrm{~Hz}, \mathrm{ArH}), 6.55$ (s, $1 \mathrm{H}, \mathrm{ArH}), 3.57$ (s, $3 \mathrm{H}, \mathrm{Me}), 1.07-0.86$ (m, $21 \mathrm{H}, \mathrm{TIPS}) .{ }^{13} \mathrm{C}$ NMR (101 $\left.\mathrm{MHz}_{\mathrm{CDCl}}\right) \delta 137.8,125.9,123.4,123.0,122.2,116.9,112.4,107.8,98.6,97.6,30.7,18.7$, 11.3; IR 2942 (m), 2891 (w), 2864 (m), 2151 (m), 1605 (w), 1464 (s), 1383 (w), 1339 (m), 1330 (m), $1229(\mathrm{w}), 997$ (w), 922 (w), 883 (s), 855 (m), 808 (s). HRMS (ESI) calcd for $\mathrm{C}_{20}{ }^{79} \mathrm{BrH}_{29} \mathrm{NSi}^{+}[\mathrm{M}+\mathrm{H}]^{+}$390.1247; found 390.1243. 


\section{7-Bromo-1-methyl-2-((triisopropylsilyl)ethynyl)-1H-indole (4g)}

Starting from 7-bromo-1-methyl-1H-indole (1g) $(105 \mathrm{mg}, 0.500 \mathrm{mmol})$,

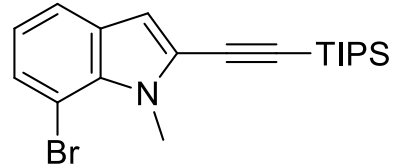

7-bromo-1-methyl-2-((triisopropylsilyl)ethynyl)-1H-indole (4g) $(141 \mathrm{mg}$, 0.361 mmol, $72 \%$ yield) was obtained as as a white solid after purification by column chromatography $\left(\mathrm{SiO}_{2}\right.$, hexane). $\mathrm{R}_{\mathrm{f}}$ : 0.95 (hexanes/EtOAc 10/1). Mp.: 39-42 ${ }^{\circ} \mathrm{C} .{ }^{1} \mathrm{H} \mathrm{NMR}\left(400 \mathrm{MHz}, \mathrm{CDCl}_{3}\right) \delta 7.47$ (dd, $1 \mathrm{H}, J=7.9,0.9$ $\mathrm{Hz}, \mathrm{ArH}), 7.37$ (dd, $1 \mathrm{H}, J=7.6,0.9 \mathrm{~Hz}, \mathrm{ArH}), 6.90$ (t, $1 \mathrm{H}, J=7.7 \mathrm{~Hz}, \mathrm{ArH}), 6.76$ (s, $1 \mathrm{H}, \operatorname{ArH})$, 4.22 (s, $3 \mathrm{H}, \mathrm{Me}), 1.05-1.23$ (m, $21 \mathrm{H}$, TIPS) ${ }^{13} \mathrm{C} \mathrm{NMR}\left(101 \mathrm{MHz}, \mathrm{CDCl}_{3}\right) \delta 133.5,130.1,128.2$, 124.7, 121.1, 120.4, 108.2, 103.8, 99.2, 97.6, 34.0, 18.7, 11.3. IR 2942 (s), 2891 (w), 2865 (s), 2153 (m), 1557 (w), 1483 (w), 1463 (m), 1449 (m), 1406 (w), 1381 (w), 1368 (w), 1347 (m), 1317 (s), 1308 (m), 1208 (w), 1162 (w), 1099 (s), 1073 (w), 1055 (w), 1017 (w), 997 (m), 922 (m), 883 (s). HRMS (ESI) calcd for $\mathrm{C}_{20}{ }^{79} \mathrm{BrH}_{29} \mathrm{NSi}^{+}[\mathrm{M}+\mathrm{H}]^{+} 390.1247$; found 390.1232 .

\section{1-Methyl-5-nitro-2-((triisopropylsilyl)ethynyl)-1H-indole (4h)}

Starting from 1-methyl-5-nitro-1H-indole (1h) $(88 \mathrm{mg}, 0.50$<smiles>Cn1c(C#C[In])cc2cc([N+](=O)[O-])ccc21</smiles>
mmol), 1-methyl-5-nitro-2-((triisopropylsilyl)ethynyl)-1 $H$-indole (4h) (86 mg, $0.24 \mathrm{mmol}, 48 \%$ yield) was obtained as a yellow solid, after purification by column chromatography $\left(\mathrm{SiO}_{2}\right.$, hexane to hexane/DCM 8/2, then hexane/EtOAc 98/2 to 95/5) Rf: 0.65 (hexanes:EtOAc 10:1). Mp.: 57$59^{\circ} \mathrm{C} .{ }^{1} \mathrm{H} \mathrm{NMR}\left(400 \mathrm{MHz}, \mathrm{CDCl}_{3}\right) \delta 8.53(\mathrm{~d}, 1 \mathrm{H}, J=2.2 \mathrm{~Hz}, \mathrm{ArH}), 8.15$ (dd, $1 \mathrm{H}, J=9.1,2.2$ $\mathrm{Hz}, \mathrm{ArH}), 7.30$ (d, $1 \mathrm{H}, J=9.1 \mathrm{~Hz}, \mathrm{ArH}), 6.94$ (s, $1 \mathrm{H}, \mathrm{ArH}), 3.88$ (s, $3 \mathrm{H}, \mathrm{Me}), 1.18$ (m, $21 \mathrm{H}$, TIPS). ${ }^{13} \mathrm{C}$ NMR $\left(101 \mathrm{MHz}, \mathrm{CDCl}_{3}\right) \delta 142.1,139.6,126.2,125.8,118.5,118.1,109.7,109.3$, 100.4, 96.4, 31.1, 18.7, 11.2, IR 2942 (w), 2890 (w), 2865 (w), 2360 (w), 2343 (w), 2154 (w), $1520(\mathrm{~m}), 1463(\mathrm{~m}), 1390(\mathrm{w}), 1348(\mathrm{~m}), 1329(\mathrm{~s}), 1068(\mathrm{~m}), 997(\mathrm{w}), 883(\mathrm{~m}), 784(\mathrm{w}), 752(\mathrm{~m})$, 721 (s), 677 (m), 662 (m), 647 (m), 617 (m), 574 (w), 512 (m), 498 (m), 491 (s), 461 (s), 445 (m), 430 (s), 403 (s). HRMS (ESI) calcd for $\mathrm{C}_{20} \mathrm{H}_{29} \mathrm{~N}_{2} \mathrm{O}_{2} \mathrm{Si}^{+}[\mathrm{M}+\mathrm{H}]^{+}$357.1993; found 357.1997.

\section{1-Methyl-6-nitro-2-((triisopropylsilyl)ethynyl)-1H-indole (4i)}

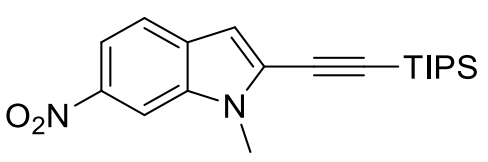

Starting from 1-methyl-6-nitro- $1 H$-indole (1i) $(88 \mathrm{mg}, 0.50 \mathrm{mmol})$, 1-methyl-6-nitro-2-((triisopropylsilyl)ethynyl)-1H-indole (4i) (81 $\mathrm{mg}, 0.23 \mathrm{mmol}, 45 \%$ yield) obtained as a brown solid after purification by column chromatography $\left(\mathrm{SiO}_{2}\right.$, hexane to hexane/DCM 70/30) Rf: 0.70 (hexanes/EtOAc 10/1). Mp.: 89-96 ${ }^{\circ} \mathrm{C} .{ }^{1} \mathrm{H}$ NMR (400 MHz, $\left.\mathrm{CDCl}_{3}\right)$ $\delta 8.18(\mathrm{~d}, 1 \mathrm{H}, J=1.8 \mathrm{~Hz}, \mathrm{ArH}), 7.93(\mathrm{dd}, 1 \mathrm{H}, J=8.8,2.0 \mathrm{~Hz}, \mathrm{ArH}), 7.53(\mathrm{~d}, 1 \mathrm{H}, J=8.8 \mathrm{~Hz}$, ArH), 6.77 (d, $1 \mathrm{H}, J=0.7 \mathrm{~Hz}, \mathrm{ArH}), 3.84$ (s, $3 \mathrm{H}, \mathrm{Me}$ ), 1.20-0.94 (m, $21 \mathrm{H}, \mathrm{TIPS}) .{ }^{13} \mathrm{C}$ NMR $\left(101 \mathrm{MHz}, \mathrm{CDCl}_{3}\right) \delta 143.9,135.6,131.8,128.0,120.8,115.5,108.0,106.4,101.5,96.5,31.1$, 18.7, 11.3. IR 2956 (m), 2941 (m), 2889 (w), 2864 (m), 2154 (w), $1751(\mathrm{w}), 1519$ (m), 1503 (s), 1463 (s), 1385 (w), 1362 (m), 1344 (s), 1330 (s), 1300 (m), 1256 (w), 1235 (m), 1234 (m), 1143 (w), 1129 (m), 1072 (m), 1063 (m), 1039 (w), 1020 (m), 1012 (m), 996 (m), 922 (w), 883 (s), 862 (w), $844(\mathrm{w}), 822$ (m). HRMS (ESI) calcd for $\mathrm{C}_{20} \mathrm{H}_{29} \mathrm{~N}_{2} \mathrm{O}_{2} \mathrm{Si}^{+}[\mathrm{M}+\mathrm{H}]^{+}$357.1993; found 357.1990. 


\section{5-Methoxy-1-methyl-2-((triisopropylsilyl)ethynyl)-1H-indole (4j)}

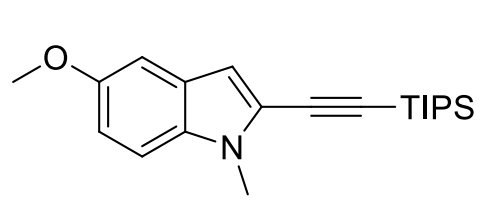

Starting from 5-methoxy-1-methyl-1H-indole (1j) $(81 \mathrm{mg}, 0.50$ mmol), 5-methoxy-1-methyl-2-((triisopropylsilyl)ethynyl)-1Hindole (4j) (94.5 mg, $0.277 \mathrm{mmol}, 55 \%$ yield) was obtained as a brownish solid, after purification by column chromatography $\left(\mathrm{SiO}_{2}\right.$, hexane to hexane/DCM 90/10) giving a highly fluorescent spot on TLC. $\mathrm{R}_{\mathrm{f}}: 0.75$ (hexanes/EtOAc 10/1). Mp.: 113-118 ${ }^{\circ} \mathrm{C} .{ }^{1} \mathrm{H}$ NMR (400 MHz, $\left.\mathrm{CDCl}_{3}\right) \delta 7.17$ (d, $1 \mathrm{H}, J=6.4 \mathrm{~Hz}$, ArH), $7.02(\mathrm{~d}, 1 \mathrm{H}, J=2.3 \mathrm{~Hz}, \operatorname{ArH}), 6.95(\mathrm{~d}, 1 \mathrm{H}, J=2.4 \mathrm{~Hz}, \operatorname{ArH}), 6.72$ (d, $1 \mathrm{H}, J=0.8 \mathrm{~Hz}$, ArH), 3.86 (s, $3 \mathrm{H}, \mathrm{Me}), 3.81$ (s, $3 \mathrm{H}, \mathrm{Me}), 1.19$ (m, $21 \mathrm{H}, \mathrm{TIPS}) .{ }^{13} \mathrm{C}$ NMR $\left(101 \mathrm{MHz}, \mathrm{CDCl}_{3}\right) \delta$ 154.5, 132.6, 127.3, 122.7, 113.9, 110.2, 107.1, 102.0, 98.3, 97.6, 55.8, 30.7, 18.7, 11.4. IR 2944 (m), $2891(\mathrm{w}), 2865$ (m), 2834 (w), 2146 (m), $1621(\mathrm{w}), 1518$ (w), $1474(\mathrm{~m}), 1464(\mathrm{~m}), 1429$ (m), 1385 (w), 1366 (w), 1349 (m), 1293 (m), 1265 (m), 1242 (m), 1215 (s), 1179 (m), 1163 (w), 1142 (m), 1101 (w), 1072 (w), 1030 (m), 1018 (w), 996 (m), 946 (w), 921 (w), 883 (s), 846 (m), 804 (m). HRMS (ESI) calcd for $\mathrm{C}_{21} \mathrm{H}_{32} \mathrm{NOSi}^{+}[\mathrm{M}+\mathrm{H}]^{+}$342.2248; found 342.2253.

\section{1-(3-Phenylpropyl)-2-((triisopropylsilyl)ethynyl)-1H-indole (4k)}

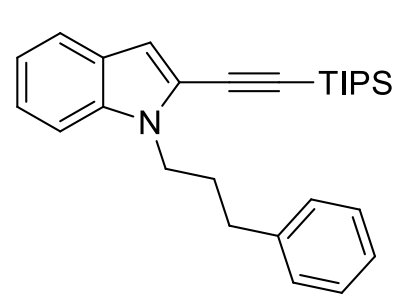

Starting from 1-(3-phenylpropyl)-1H-indole (1k) $(0.118 \mathrm{~g}, \quad 0.500$ mmol), 1-(3-phenylpropyl)-2-((triisopropylsilyl)ethynyl)-1 $H$-indole (4k) $(0.120 \mathrm{~g}, 0.289 \mathrm{mmol}, 58 \%$ yield) was obtained as a yellow oil after purification by column chromatography $\left(\mathrm{SiO}_{2}\right.$, hexane to hexane/DCM 80/20). Rf: 0.75 (hexanes:EtOAc 10:1). ${ }^{1} \mathrm{H}$ NMR (400 $\left.\mathrm{MHz}, \mathrm{CDCl}_{3}\right) \delta 7.38(\mathrm{dd}, 1 \mathrm{H}, J=7.9,0.8 \mathrm{~Hz}, \mathrm{ArH}), 7.12-6.88(\mathrm{~m}, 8 \mathrm{H}$, ArH), 6.62 (d, $1 \mathrm{H}, J=0.6 \mathrm{~Hz}, \mathrm{ArH}), 4.12\left(\mathrm{t}, 2 \mathrm{H}, J=7.3 \mathrm{~Hz}, \mathrm{CH}_{2}\right), 2.52-2.46\left(\mathrm{~m}, 2 \mathrm{H}, \mathrm{CH}_{2}\right.$ ), $1.97\left(\mathrm{~m}, 2 \mathrm{H}, \mathrm{CH}_{2}\right), 1.10-0.85$ (m, $\left.21 \mathrm{H}, \mathrm{TIPS}\right) .{ }^{13} \mathrm{C} \mathrm{NMR}\left(101 \mathrm{MHz}, \mathrm{CDCl}_{3}\right) \delta 154.6,137.7$, 132.0, 128.6, 127.7, 127.3, 126.7, 122.5, 114.0, 110.8, 107.8, 102.1, 98.2, 98.0, 44.3, 33.4, 31.8, 18.8, 11.4. IR 3057 (w), 2942 (s), 2865 (s), 2361 (w), 2341 (w), 2152 (m), 1520 (w), 1458 (s), 1388 (m), 1346 (m), 1216 (w), 1166 (w), 1073 (w), 996 (m), 922 (m), 883 (s), 851 (m). HRMS (ESI) calcd for $\mathrm{C}_{28} \mathrm{H}_{38} \mathrm{NSi}^{+}[\mathrm{M}+\mathrm{H}]^{+} 416.2768$; found 416.2762.

\section{1-Benzyl-5-methoxy-2-((triisopropylsilyl)ethynyl)-1H-indole (4l)}

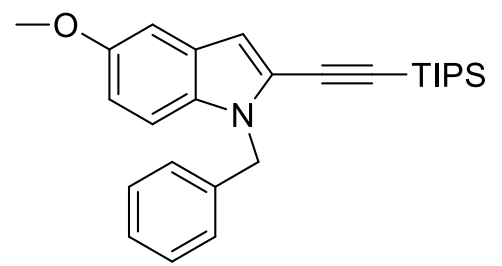

Starting from 1-benzyl-5-methoxy-1H-indole (11) (119 mg, 0.500 mmol), 1-benzyl-5-methoxy-2-((triisopropylsilyl)ethynyl)-1Hindole (4I) (86 mg, $0.21 \mathrm{mmol}, 41 \%$ yield) was obtained as a redish-brown oil after purification by column chromatography ( $\mathrm{SiO}_{2}$, hexane to hexane/DCM 90/10). $\mathrm{R}_{\mathrm{f}}$ : 0.70 (hexanes:EtOAc 10:1), 0.35 (hexanes:EtOAc 20:1). ${ }^{1} \mathrm{H}$ NMR (400 $\left.\mathrm{MHz} \mathrm{CDCl}_{3}\right) \delta$ 7.16-6.95 (m, $\left.6 \mathrm{H}, \mathrm{ArH}\right), 6.90$ (d, $1 \mathrm{H}, J=2.3 \mathrm{~Hz}, \mathrm{ArH}), 6.73(\mathrm{dd}, 1 \mathrm{H}, J=8.9,2.5 \mathrm{~Hz}, \mathrm{ArH}), 6.66$ (d, $1 \mathrm{H}, J=0.8 \mathrm{~Hz}, \operatorname{ArH})$, 5.30 (s, $2 \mathrm{H}, \mathrm{Bz} \mathrm{CH}_{2}$ ), 3.72 (s, $\left.3 \mathrm{H}, \mathrm{OMe}\right), 1.10-0.89$ (m, $\left.21 \mathrm{H}, \mathrm{TIPS}\right) .{ }^{13} \mathrm{C} \mathrm{NMR}(101 \mathrm{MHz}$, $\left.\mathrm{CDCl}_{3}\right) \delta 154.6,137.7,132.0,128.6,127.7,127.3,126.7,122.5,114.1,110.8,107.9,102.1,98.3$, 98.1, 55.8, 48.1, 18.7, 11.3; IR 2942 (m), 2891 (w), 2864 (m), 2148 (m), 1789 (w), $1624(\mathrm{w})$, 1475 (s), 1453 (s), 1392 (m), 1346 (m), 1294 (m), 1222 (s), 1175 (m), 1134 (w), 1113 (m), 1037 (m), $997(\mathrm{~m}), 883(\mathrm{~s}), 836(\mathrm{~m})$. HRMS (ESI) calcd for $\mathrm{C}_{27} \mathrm{H}_{36} \mathrm{NOSi}^{+}[\mathrm{M}+\mathrm{H}]^{+}$418.2561; found 418.2563. 


\section{1-Benzyl-2-((triisopropylsilyl)ethynyl)-1H-indole (4m)}

Starting from 1-benzyl-1H-indole (1m) $(104 \mathrm{mg}, 0.500 \mathrm{mmol}), 1$ -

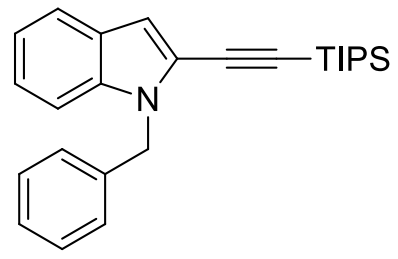

Benzyl-2-((triisopropylsilyl)ethynyl)- $1 H$-indole (4m) $(79.8 \mathrm{mg}, 0.205$

mmol, $41 \%$ yield) was obtained as a brownish oil after purification by column chromatography $\left(\mathrm{SiO}_{2}\right.$, hexane to hexane/DCM 90/10). $\mathrm{R}_{\mathrm{f}}$ : 0.65 (hexanes:EtOAc 20:1). ${ }^{1} \mathrm{H}$ NMR $\left(400 \mathrm{MHz}, \mathrm{CDCl}_{3}\right) \delta 7.50(\mathrm{~d}, 1$ $\mathrm{H}, J=7.9 \mathrm{~Hz}, \mathrm{ArH}), 7.19-6.98(\mathrm{~m}, 8 \mathrm{H}, \mathrm{ArH}), 6.78(\mathrm{~s}, 1 \mathrm{H}, \mathrm{ArH})$, 5.37 (s, $\left.2 \mathrm{H}, \mathrm{Bz} \mathrm{CH}_{2}\right), 1.07-0.92(\mathrm{~m}, 21 \mathrm{H}, \mathrm{TIPS}) .{ }^{13} \mathrm{C} \mathrm{NMR}\left(101 \mathrm{MHz}, \mathrm{CDCl}_{3}\right) \delta 137.6,136.7$, 128.6, 127.3, 126.8, 123.3, 122.2, 121.2, 120.3, 110.0, 108.4, 98.2, 98.2, 47.9, 18.7, 11.3. IR 3062 (w), 3032 (w), 2942 (s), 2891 (m), 2864 (s), 2150 (m), 1606 (w), 1455 (s), 1388 (m), 1342 (s), $1316(\mathrm{~m}), 1213$ (w), $1163(\mathrm{w}), 1075(\mathrm{w}), 999$ (m), 919 (w), 883 (s), 851 (w). ${ }^{28}$ HRMS (ESI) calcd for $\mathrm{C}_{26} \mathrm{H}_{34} \mathrm{NSi}^{+}[\mathrm{M}+\mathrm{H}]^{+}$388.2455; found 388.2436.

\section{1-Allyl-2-((triisopropylsilyl)ethynyl)-1H-indole (4n)}

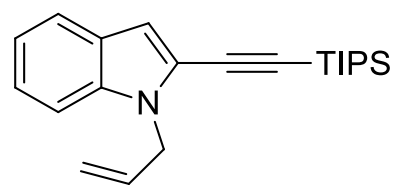

Starting from 1-allyl-1H-indole (1n) $(79 \mathrm{mg}, 0.50 \mathrm{mmol})$, 1-allyl-2((triisopropylsilyl)ethynyl)- $1 H$-indole (4n) $(74 \mathrm{mg}, 0.22 \mathrm{mmol}, 44 \%$ yield) was obtained as a yellow oil after purification by column chromatography $\left(\mathrm{SiO}_{2}\right.$, hexane). $\mathrm{R}_{\mathrm{f}}$ : 0.70 (hexanes:EtOAc 20:1) ${ }^{1} \mathrm{H}$ NMR (400 MHz, $\left.\mathrm{CDCl}_{3}\right) \delta 7.41(\mathrm{dt}, 1 \mathrm{H}, J=8.0,0.9 \mathrm{~Hz}, \mathrm{ArH}), 7.10-$ $7.03(\mathrm{~m}, 2 \mathrm{H}, \mathrm{ArH}), 6.93(\mathrm{~m}, 1 \mathrm{H}, \mathrm{ArH}), 6.64(\mathrm{~m}, 1 \mathrm{H}, \mathrm{ArH}), 5.82-5.73(\mathrm{~m}, 1 \mathrm{H}$, allyl H), 4.97 (dq, $1 \mathrm{H}, J=10.3,1.5 \mathrm{~Hz}$, allyl $\left.\mathrm{CH}_{2}\right), 4.82(\mathrm{~m}, 1 \mathrm{H}$, allyl $\mathrm{CH}), 4.70(\mathrm{dt}, 2 \mathrm{H}, J=5.1,1.7 \mathrm{~Hz}$, allyl $\left.\mathrm{CH}_{2}\right), 1.06-0.81\left(\mathrm{~m}, 21 \mathrm{H}\right.$, TIPS). ${ }^{13} \mathrm{C}$ NMR $\left(101 \mathrm{MHz}, \mathrm{CDCl}_{3}\right) \delta 136.5,133.1,127.2,123.1$, 121.8, 121.1, 120.2, 116.7, 109.9, 108.1, 97.9, 97.8, 46.7, 18.7, 11.4; IR 2942 (s), 2891 (m), 2865 (s), $2361(\mathrm{w}), 2341(\mathrm{w}), 2150(\mathrm{~s}), 1458(\mathrm{~s}), 1388(\mathrm{~m}), 1343(\mathrm{~m}), 1316(\mathrm{~m}), 1216(\mathrm{w}), 1165(\mathrm{w})$, 1073 (w), 996 (m), 922 (m), 883 (s), 851 (m). HRMS (ESI) calcd for $\mathrm{C}_{22} \mathrm{H}_{32} \mathrm{NSi}^{+}[\mathrm{M}+\mathrm{H}]^{+}$ 338.2299; found 338.2300.

\section{2-((Triisopropylsilyl)ethynyl)-1-(2-((triisopropylsilyl)oxy)ethyl)-1H-indole (40)}

Starting from 1-(2-((triisopropylsilyl)oxy)ethyl)- $1 H$-indole (1o) (159

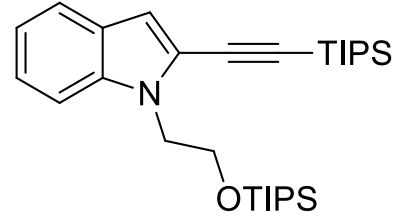

$\mathrm{mg}, \quad 0.500 \quad \mathrm{mmol}), \quad 2-(($ triisopropylsilyl)ethynyl)-1-(2((triisopropylsilyl) oxy)ethyl)-1 $H$-indole $(\mathbf{4 o})(170 \mathrm{mg}, 0.341 \mathrm{mmol}$, $68 \%$ yield) was obtained as a colorless oil after purification by column chromatography $\left(\mathrm{SiO}_{2}\right.$, hexane to hexane/DCM 90/10). $\mathrm{R}_{\mathrm{f}}$ : 0.70 (hexanes:EtOAc 20:1). ${ }^{1} \mathrm{H}$ NMR $\left(400 \mathrm{MHz}, \mathrm{CDCl}_{3}\right) \delta 7.36(\mathrm{~d}, 1 \mathrm{H}, J=7.9 \mathrm{~Hz}, \mathrm{ArH}), 7.17$ (dd, $1 \mathrm{H}, J=8.3,0.7 \mathrm{~Hz}, \mathrm{ArH}), 7.06-7.01(\mathrm{~m}, 1 \mathrm{H}, \mathrm{ArH}), 6.92-6.87(\mathrm{~m}, 1 \mathrm{H}, \mathrm{ArH}), 6.61(\mathrm{~d}, 1$ $\mathrm{H}, J=0.7 \mathrm{~Hz}, \mathrm{ArH}), 4.22\left(\mathrm{t}, 2 \mathrm{H}, J=6.3 \mathrm{~Hz}, \mathrm{CH}_{2}\right), 3.85\left(\mathrm{t}, 2 \mathrm{H}, J=6.2 \mathrm{~Hz}, \mathrm{CH}_{2}\right), 1.03-0.94(\mathrm{~m}$, 21H, TIPS), 0.87-0.67 (m, $21 \mathrm{H}$, TIPS). ${ }^{13} \mathrm{C} \mathrm{NMR}\left(101 \mathrm{MHz}, \mathrm{CDCl}_{3}\right) \delta 137.3,127.0,123.0$, 121.5, 120.9, 120.0, 110.1, 108.3, 98.3, 97.6, 62.7, 46.7, 18.7, 17.9, 11.9, 11.4; IR 2942 (s), 2891 (w), $2865(\mathrm{~s}), 2150(\mathrm{~m}), 1459(\mathrm{~m}), 1388(\mathrm{w}), 1366(\mathrm{w}), 1344(\mathrm{w}), 1318(\mathrm{w}), 1250(\mathrm{w}), 1166(\mathrm{w})$, $1123(\mathrm{~m}), 1072(\mathrm{w}), 1015$ (w), 997 (m), 936 (w), 921 (w), 882 (s). HRMS (ESI) calcd for $\mathrm{C}_{30} \mathrm{H}_{52} \mathrm{NOSi}_{2}{ }^{+}[\mathrm{M}+\mathrm{H}]^{+} 498.3582$; found 498.3589 .

\footnotetext{
${ }^{28}$ One carbon signal could not be resolved.
} 


\section{1-(2-Bromoethyl)-2-((triisopropylsilyl)ethynyl)-1 $H$-indole (4p)}

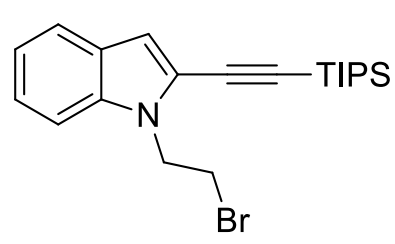

Starting from 1-(2-bromoethyl)-1 $H$-indole (1p) $(112 \mathrm{mg}, 0.500 \mathrm{mmol})$, 1-(2-bromoethyl)-2-((triisopropylsilyl)ethynyl)-1H-indole (4p) (124 $\mathrm{mg}, 0.307 \mathrm{mmol}, 61 \%$ yield) was obtained as a colorless oil after purification by column chromatography $\left(\mathrm{SiO}_{2}\right.$, hexane to hexane/DCM 90/10). Rf: 0.65 (hexanes:EtOAc 20:1), Rf: 0.30 (hexanes). ${ }^{1} \mathrm{H}$ NMR $\left(400 \mathrm{MHz}, \mathrm{CDCl}_{3}\right) \delta 7.38(\mathrm{~d}, 1 \mathrm{H}, J=7.9 \mathrm{~Hz}, \mathrm{ArH}), 7.13-7.03(\mathrm{~m}, 2 \mathrm{H}, \mathrm{ArH}), 6.93(\mathrm{~m}, 1 \mathrm{H}$, ArH), $6.63(\mathrm{~s}, 1 \mathrm{H}, \mathrm{ArH}), 4.41\left(\mathrm{t}, 2 \mathrm{H}, J=7.7 \mathrm{~Hz}, \mathrm{CH}_{2}\right), 3.41\left(\mathrm{t}, 2 \mathrm{H}, J=7.8 \mathrm{~Hz}, \mathrm{CH}_{2}\right), 1.10-0.77$ (m, $21 \mathrm{H}, \mathrm{TIPS}) .{ }^{13} \mathrm{C}$ NMR $\left(101 \mathrm{MHz}, \mathrm{CDCl}_{3}\right) \delta 136.3,127.2,123.6,121.4,121.3,120.6,109.3$, 108.9, 98.7, 97.4, 45.7, 28.6, 18.8, 11.4. IR 3059 (w), 2942 (s), 2890 (m), 2864 (s), 2150 (m), 1480 (w), 1457 (s), 1437 (w), $1386(\mathrm{~m}), 1367$ (m), 1340 (s), 1316 (m), $1281(\mathrm{w}), 1233(\mathrm{w}), 1219$ (m), 1177 (w), 1160 (m), 1073 (w), 1016 (m), 997 (m), 919 (w), 882 (s), 857 (m), 843 (w). HRMS (ESI) calcd for $\mathrm{C}_{21}{ }^{79} \mathrm{BrH}_{31} \mathrm{NSi}^{+}[\mathrm{M}+\mathrm{H}]^{+}$404.1404; found 404.1389.

\section{1-((1,3-Dioxolan-2-yl)methyl)-2-((triisopropylsilyl)ethynyl)-1H-indole (4q)}

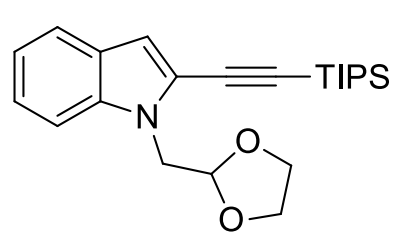

Starting from 1-((1,3-dioxolan-2-yl)methyl)-1H-indole (1q) $(102 \mathrm{mg}$, $0.500 \quad \mathrm{mmol}), \quad 1$-((1,3-dioxolan-2-yl)methyl)-2((triisopropylsilyl)ethynyl)-1H-indole (4q) (126 mg, $0.328 \mathrm{mmol}, 66 \%$ yield) was obtained as a brown oil after purification by column chromatography $\left(\mathrm{SiO}_{2}\right.$, hexane to hexane/DCM 80/20). $\mathrm{R}_{\mathrm{f}}: 0.40$ (hexanes:EtOAc 20:1) ${ }^{1} \mathrm{H}$ NMR $\left(400 \mathrm{MHz}, \mathrm{CDCl}_{3}\right) \delta 7.45(\mathrm{~d}, 1 \mathrm{H}, J=7.9 \mathrm{~Hz}, \mathrm{ArH}), 7.31(\mathrm{~m}, 1$ $\mathrm{H}, \mathrm{ArH}), 7.19-7.12(\mathrm{~m}, 1 \mathrm{H}, \mathrm{ArH}), 7.04-6.98(\mathrm{~m}, 1 \mathrm{H}, \mathrm{ArH}), 6.72(\mathrm{~s}, 1 \mathrm{H}, \mathrm{ArH}), 5.16$ (t, $1 \mathrm{H}, J=$ 4.2 Hz, $\mathrm{CH}_{2}-\mathrm{CH}-\mathrm{O}$ ), 4.36-4.28 (m, $\left.2 \mathrm{H}, \mathrm{N}-\mathrm{CH}_{2}-\mathrm{CH}\right), 3.75$ (m, $\left.4 \mathrm{H},-\mathrm{CH}_{2}-\mathrm{CH}_{2}-\right)$, 1.25-0.76 (m, 21 H, TIPS). ${ }^{13} \mathrm{C}$ NMR $\left(101 \mathrm{MHz}, \mathrm{CDCl}_{3}\right) \delta 137.2,127.2,123.3,122.2,121.0,120.3,110.3,108.7$, 102.6, 98.1, 97.9, 65.2, 47.5, 18.7, 11.4. IR $3058(\mathrm{w}), 2942(\mathrm{~m}), 2890(\mathrm{~m}), 2864(\mathrm{~s}), 2150(\mathrm{~m})$, 1480 (w), 1457 (s), 1437 (w), 1386 (m), 1367 (m), 1340 (s), 1315 (m), 1281 (w), 1233 (w), 1219 (m), 1177 (w), 1161 (m), 1160 (m), 1073 (w), 1016 (w), 997 (m), 916 (w), 882 (s), 857 (m), 843 (w). HRMS (ESI) calcd for $\mathrm{C}_{23} \mathrm{H}_{34} \mathrm{NO}_{2} \mathrm{Si}^{+}[\mathrm{M}+\mathrm{H}]^{+} 384.2353$; found 384.2357 . 


\section{Spectra}

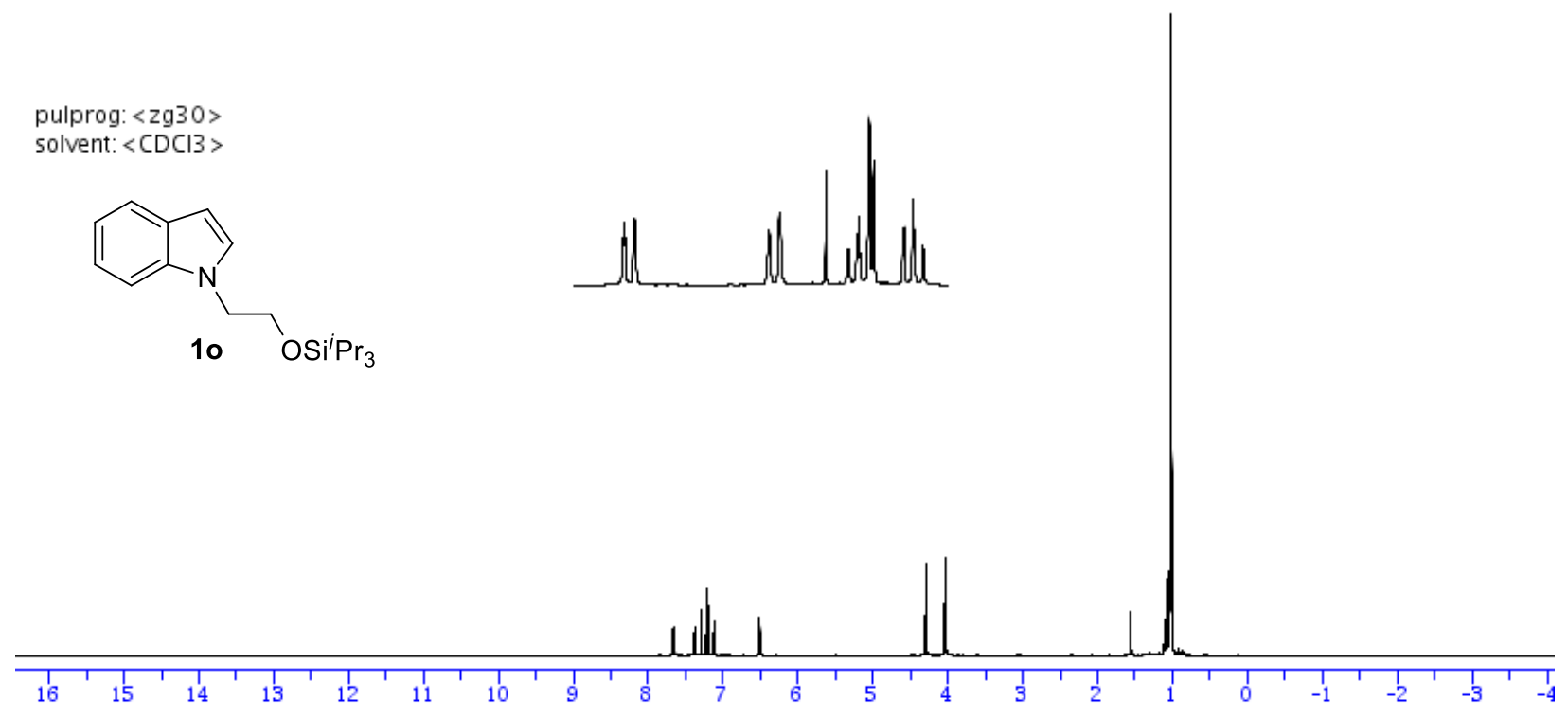

pulprog: $\langle$ zgpg30 >

solvent: $\langle\mathrm{CDCl} 3\rangle$
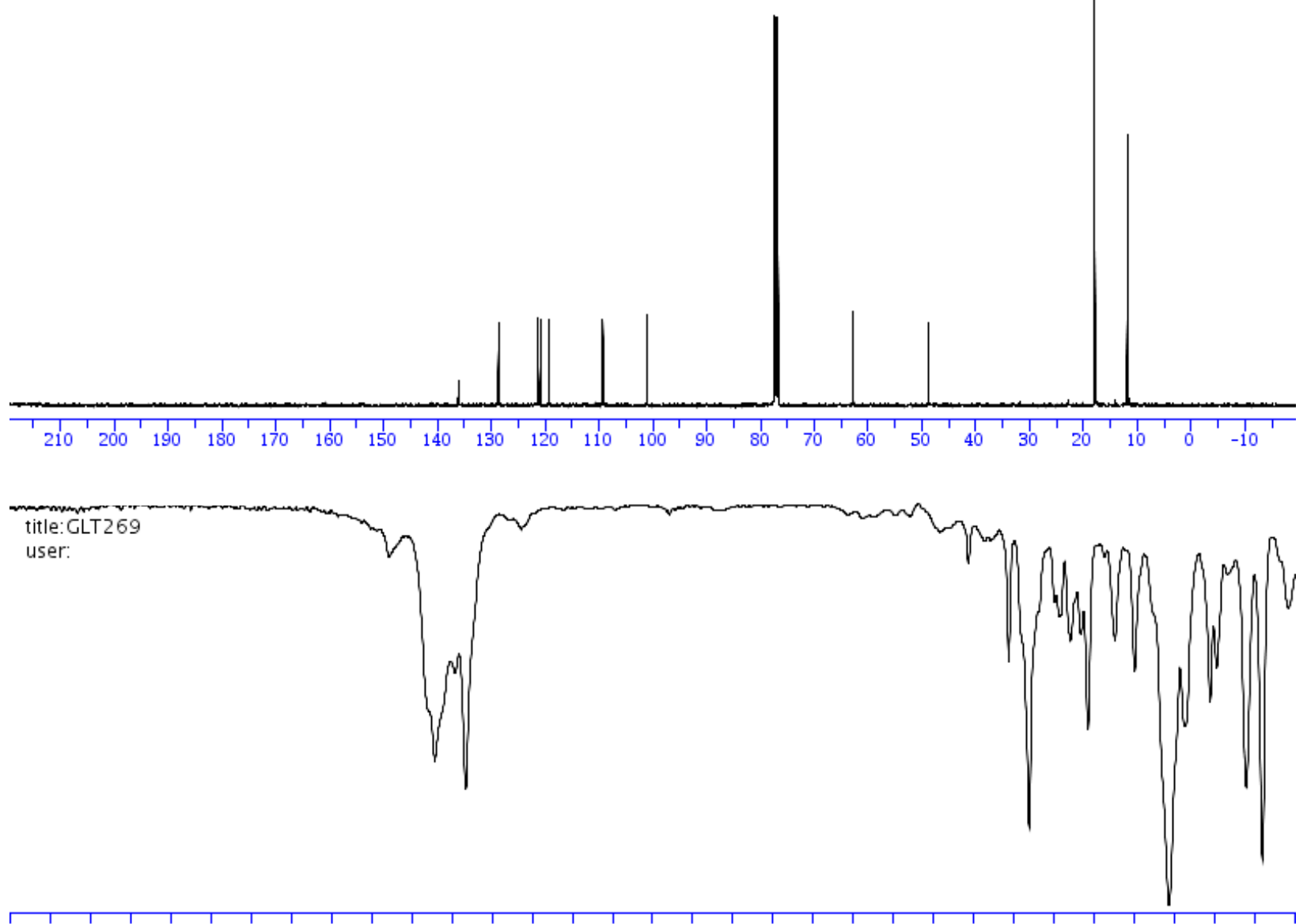
pulprog: $\langle 2 \mathrm{~g} 30\rangle$

solvent: $\langle\mathrm{CDCI} 3\rangle$

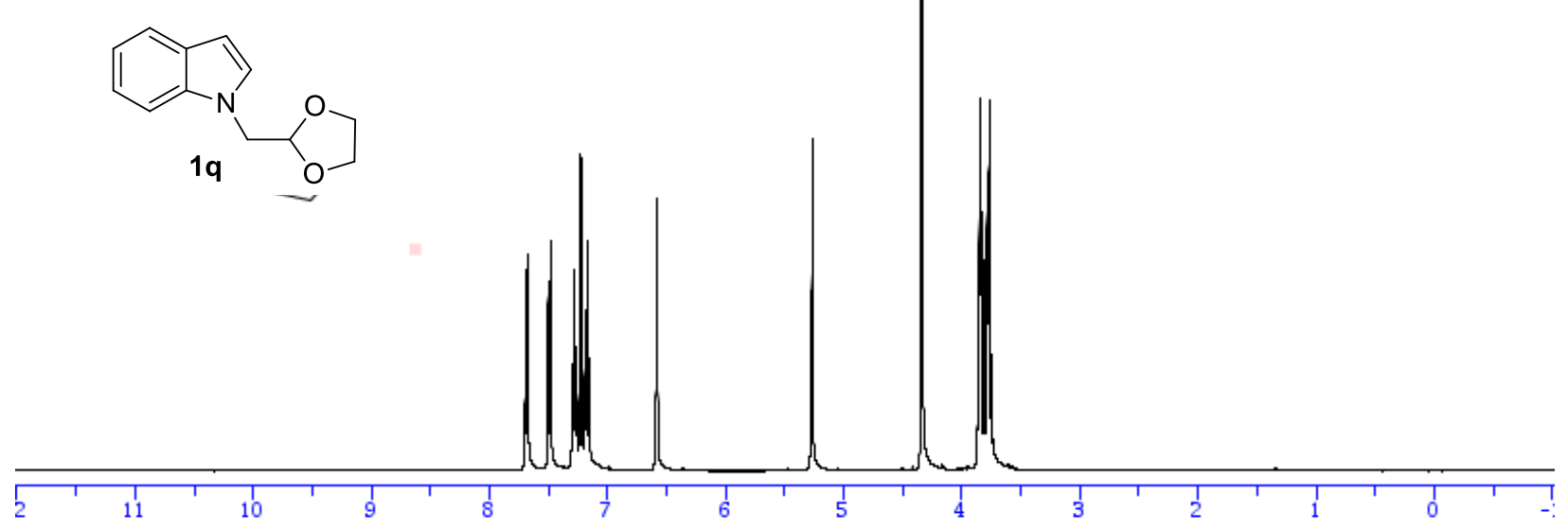

.... $-\cdots,-\cdots$,

pulprog: $\langle z g p g 30\rangle$

solvent: $\langle\mathrm{CDCI} 3\rangle$

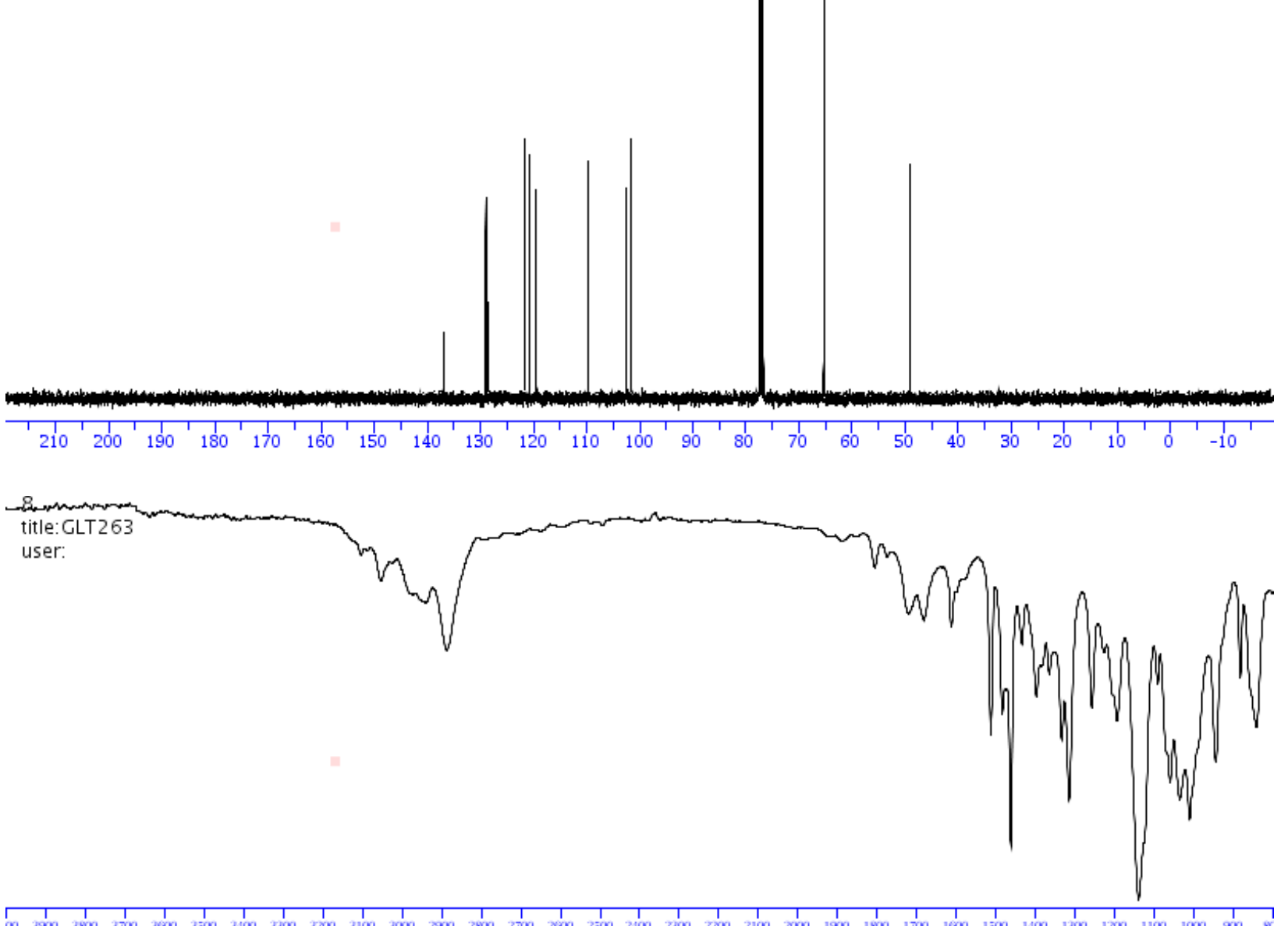




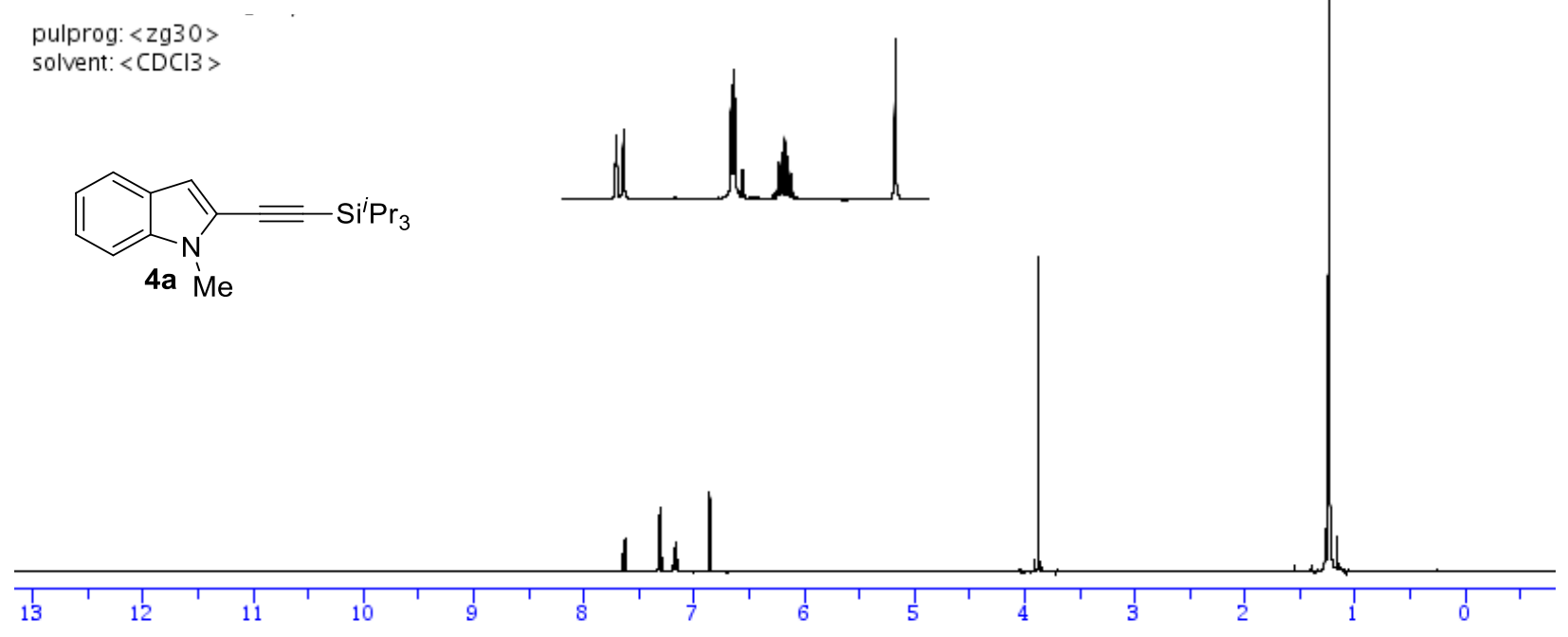

solvent: $\langle\mathrm{CDCI}\rangle$
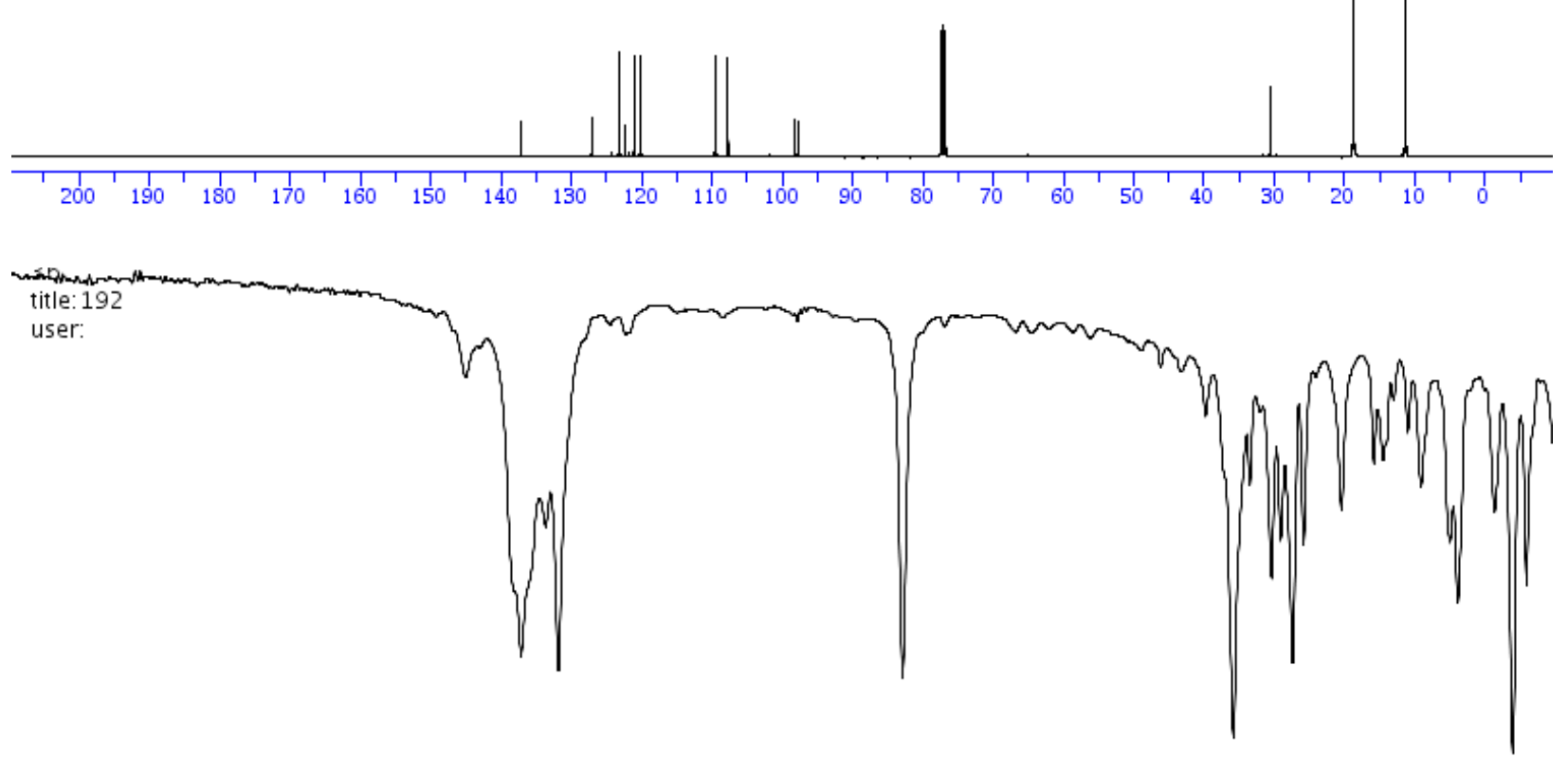

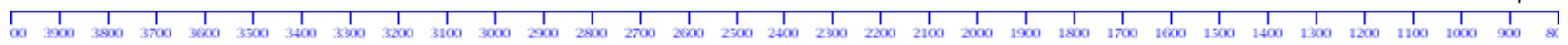



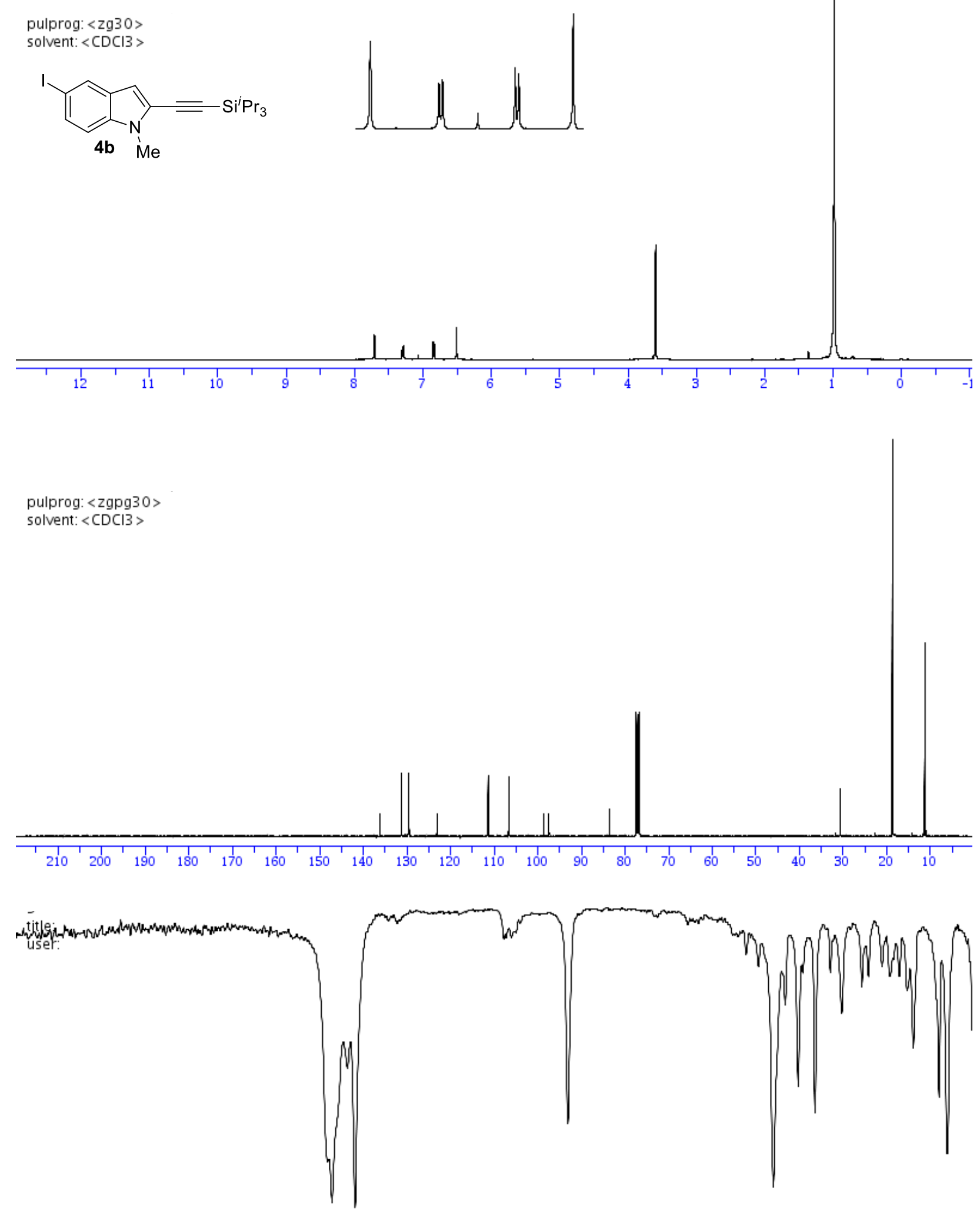

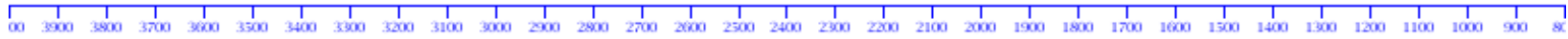



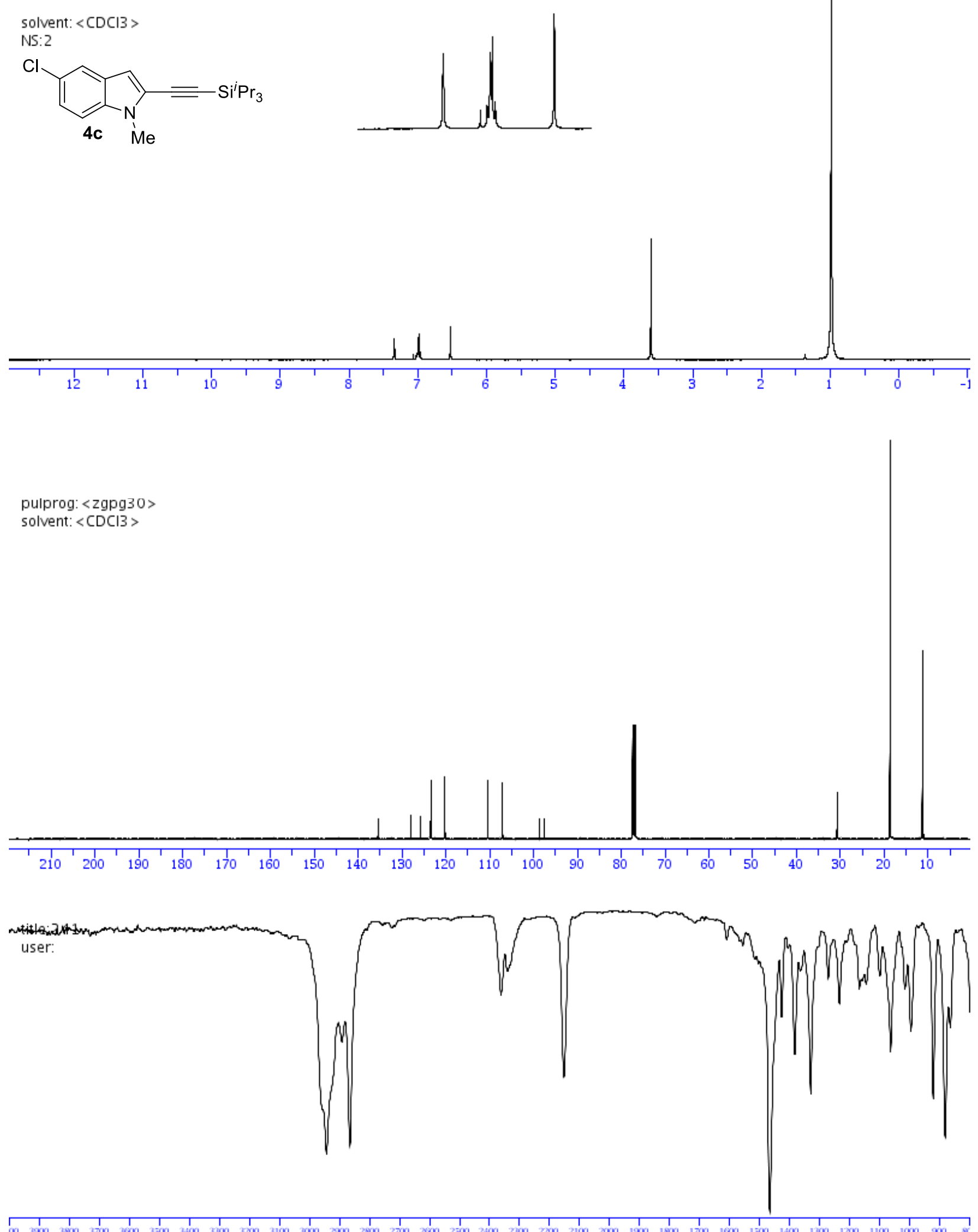

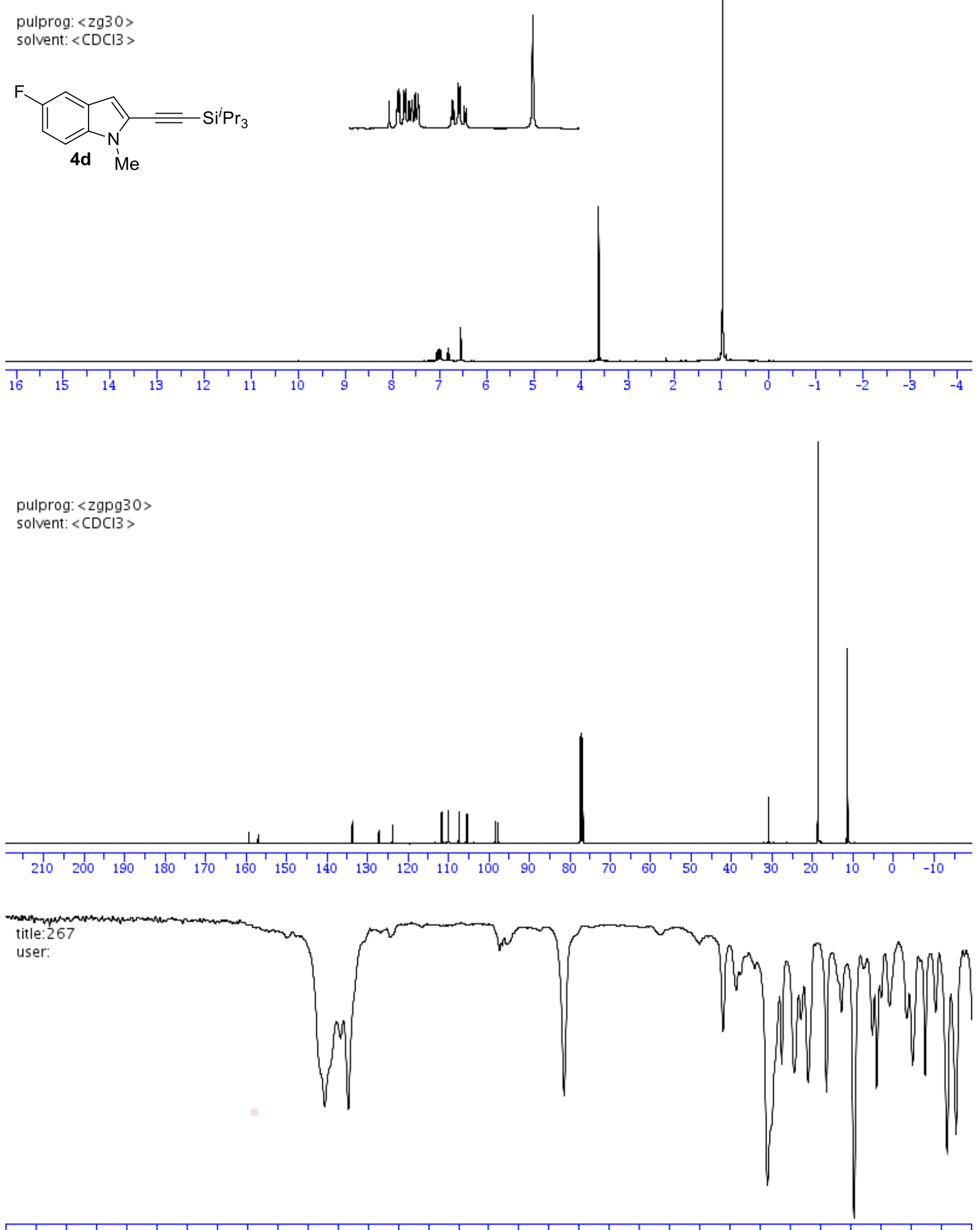

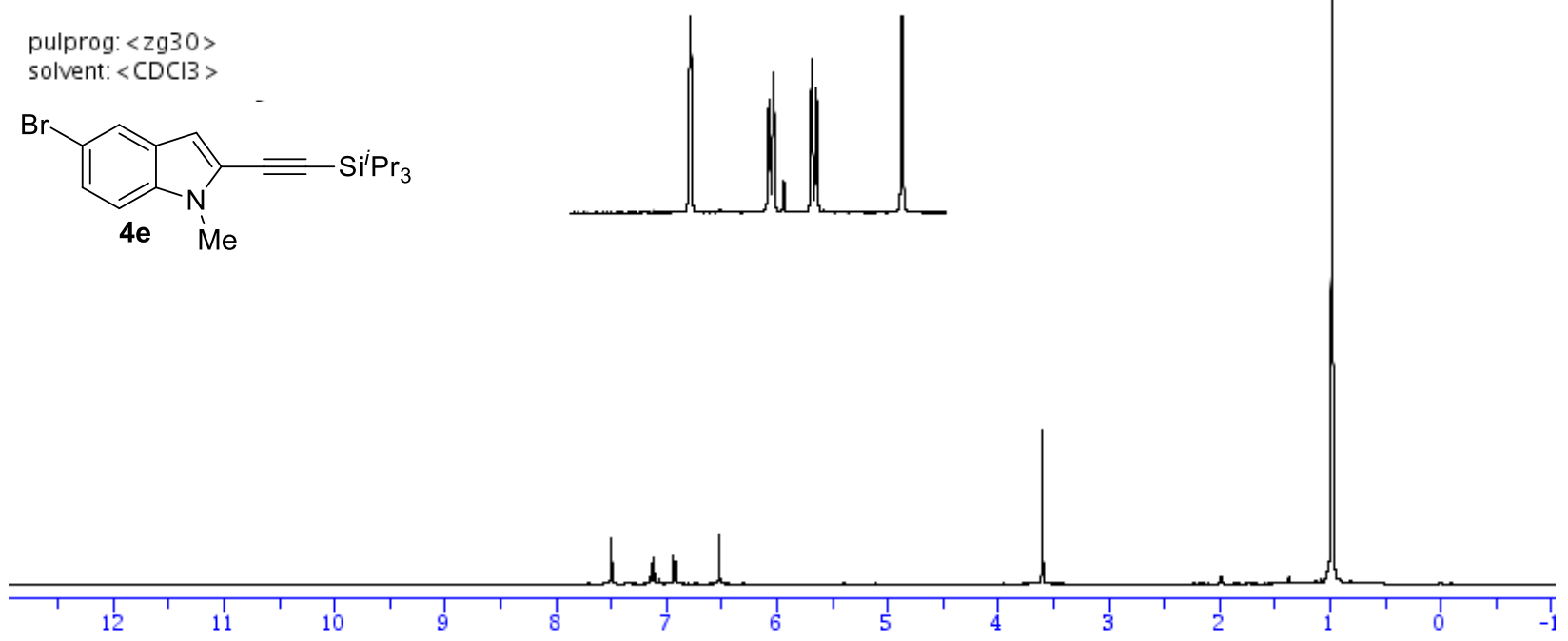

pulprog: $\langle$ zgpg30 >

solvent: $\langle\mathrm{CDCl} 3\rangle$
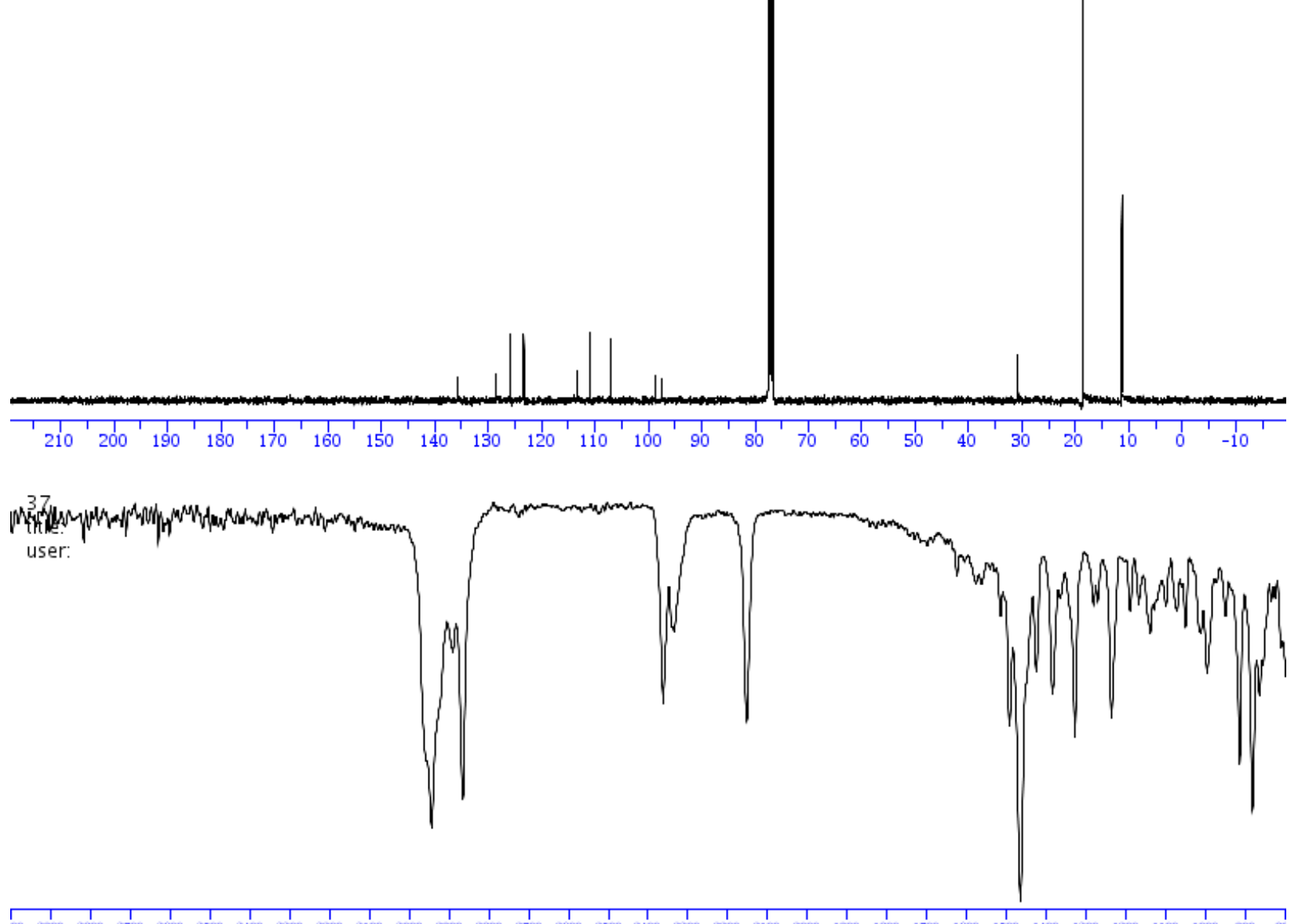

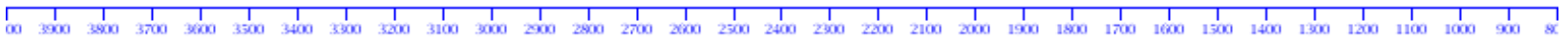


pulprog: $\langle 2 g 30\rangle$

enkmnt. , rnris -

$\mathrm{Si}_{4 \mathrm{i}} \mathrm{Pr}_{3}$
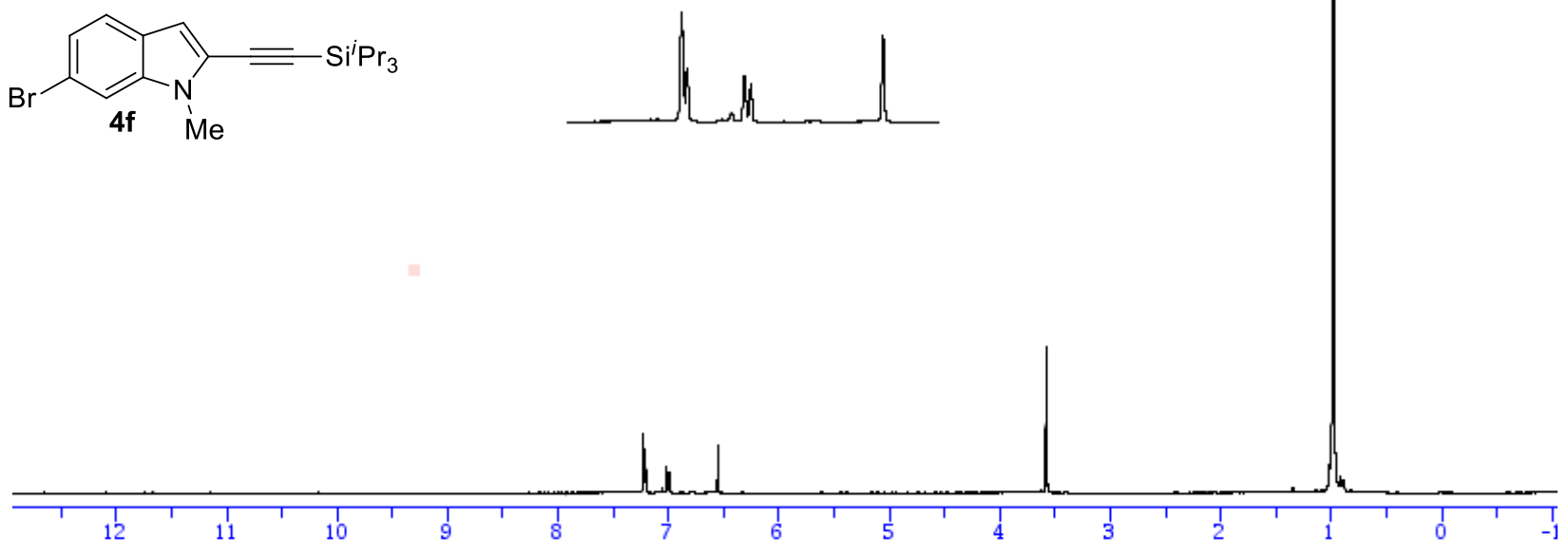

pulprog: $\langle$ zgpg30>

solvent: $\langle\mathrm{CDCI} 3\rangle$
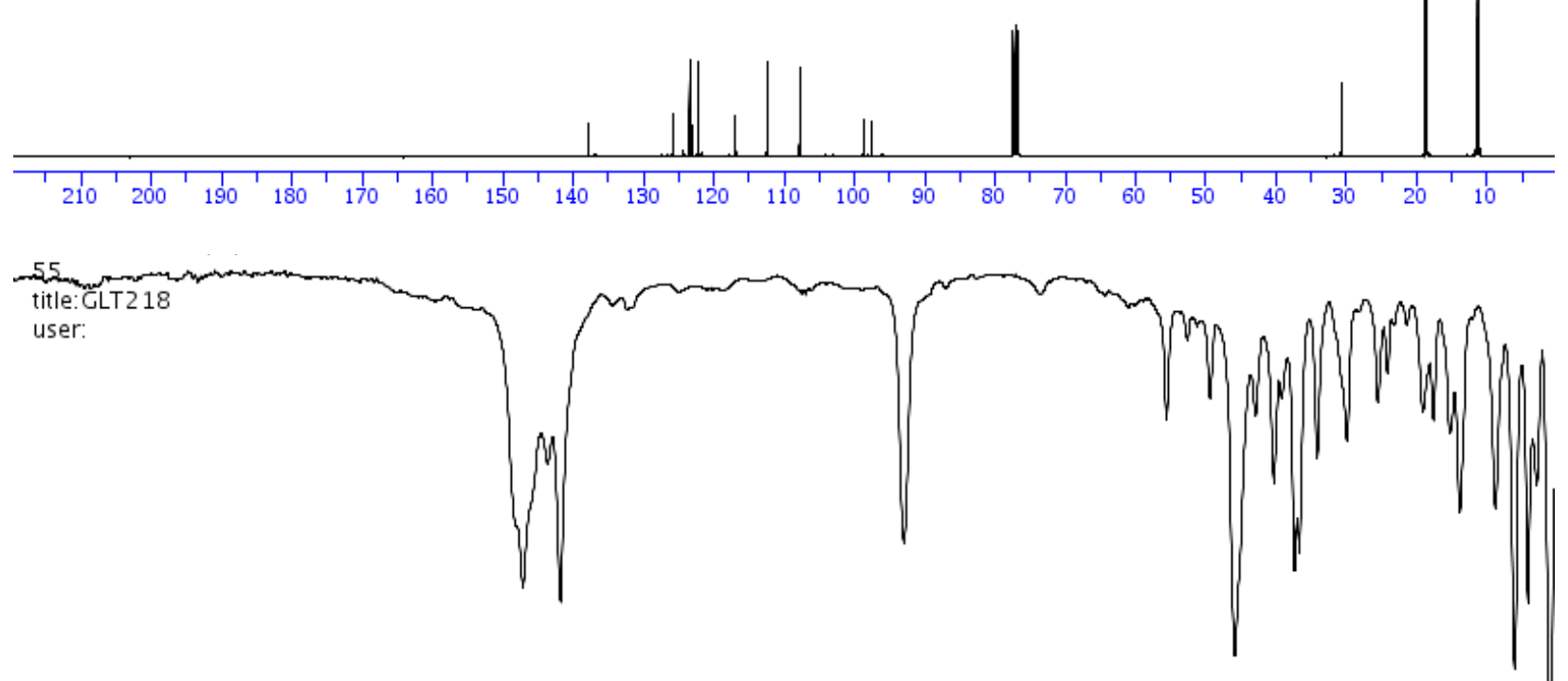

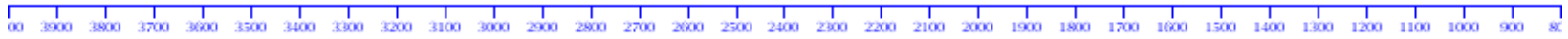


pulprog: $\langle z \mathrm{~g} 30\rangle$

solvent: $\langle\mathrm{CDCl} 3\rangle$<smiles>Cn1c(C#C[SiH2][18F])cc2cccc(Br)c21</smiles>

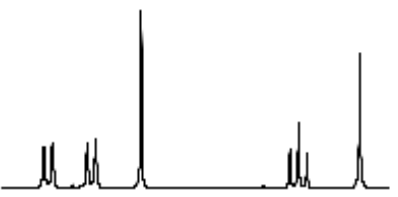

pulprog: <zgpg30 >

solvent: $\langle\mathrm{CDCl} 3\rangle$
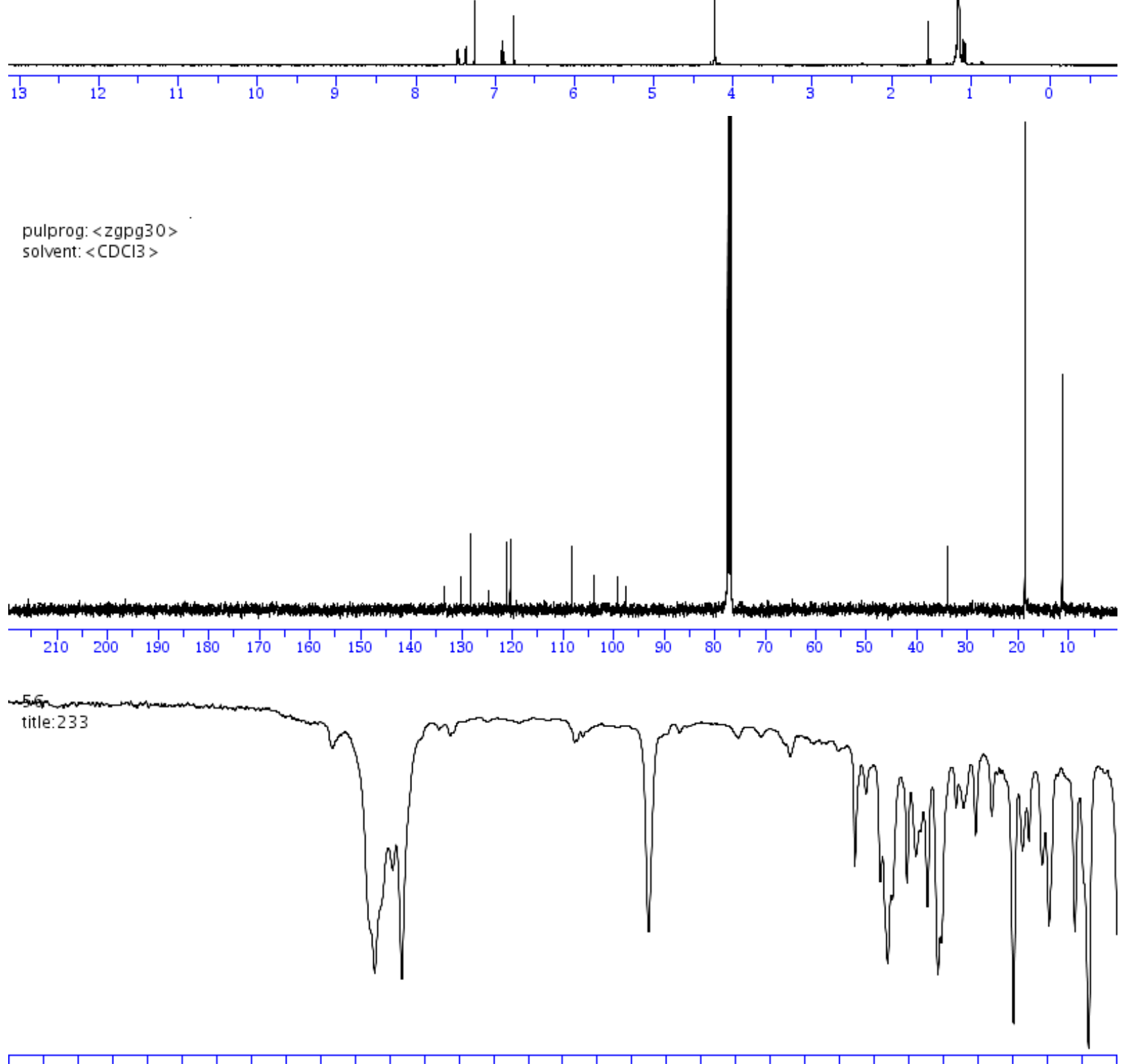

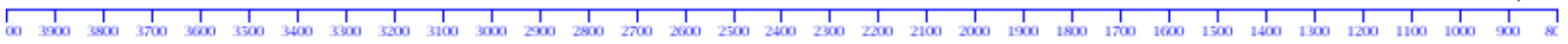



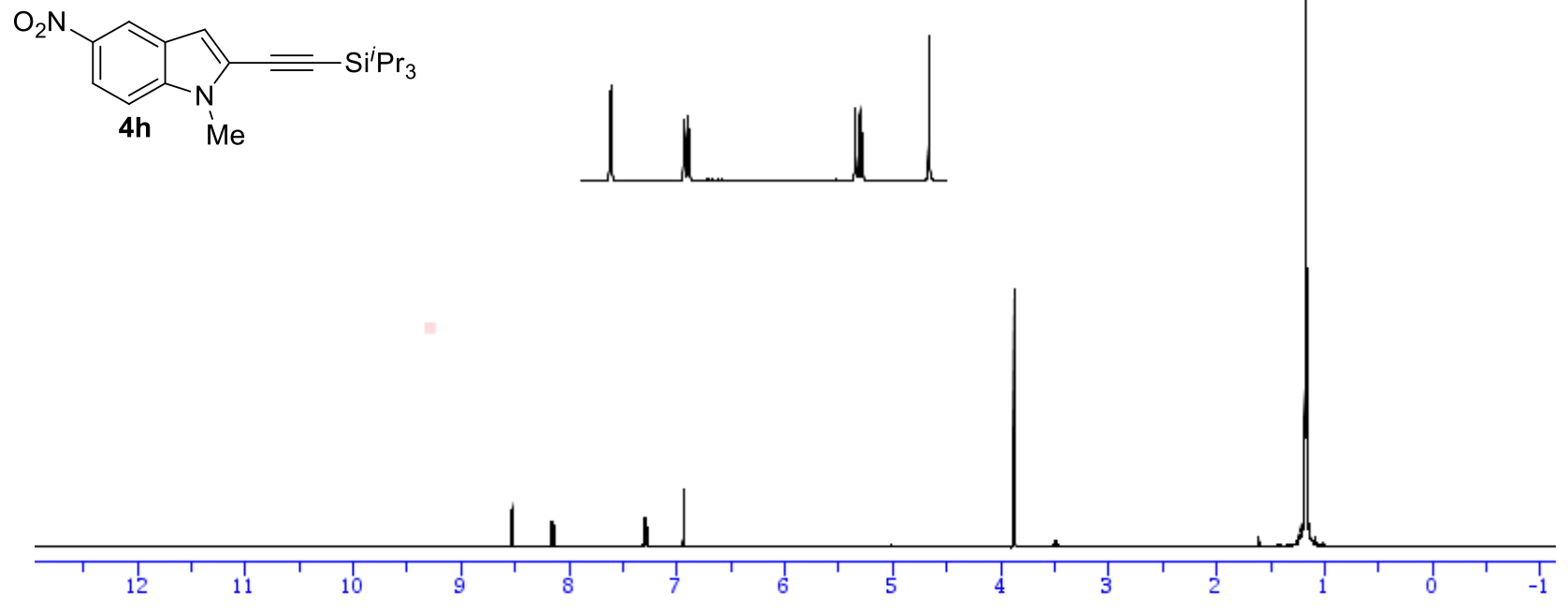

pulprog: $\langle z g p g\rangle$
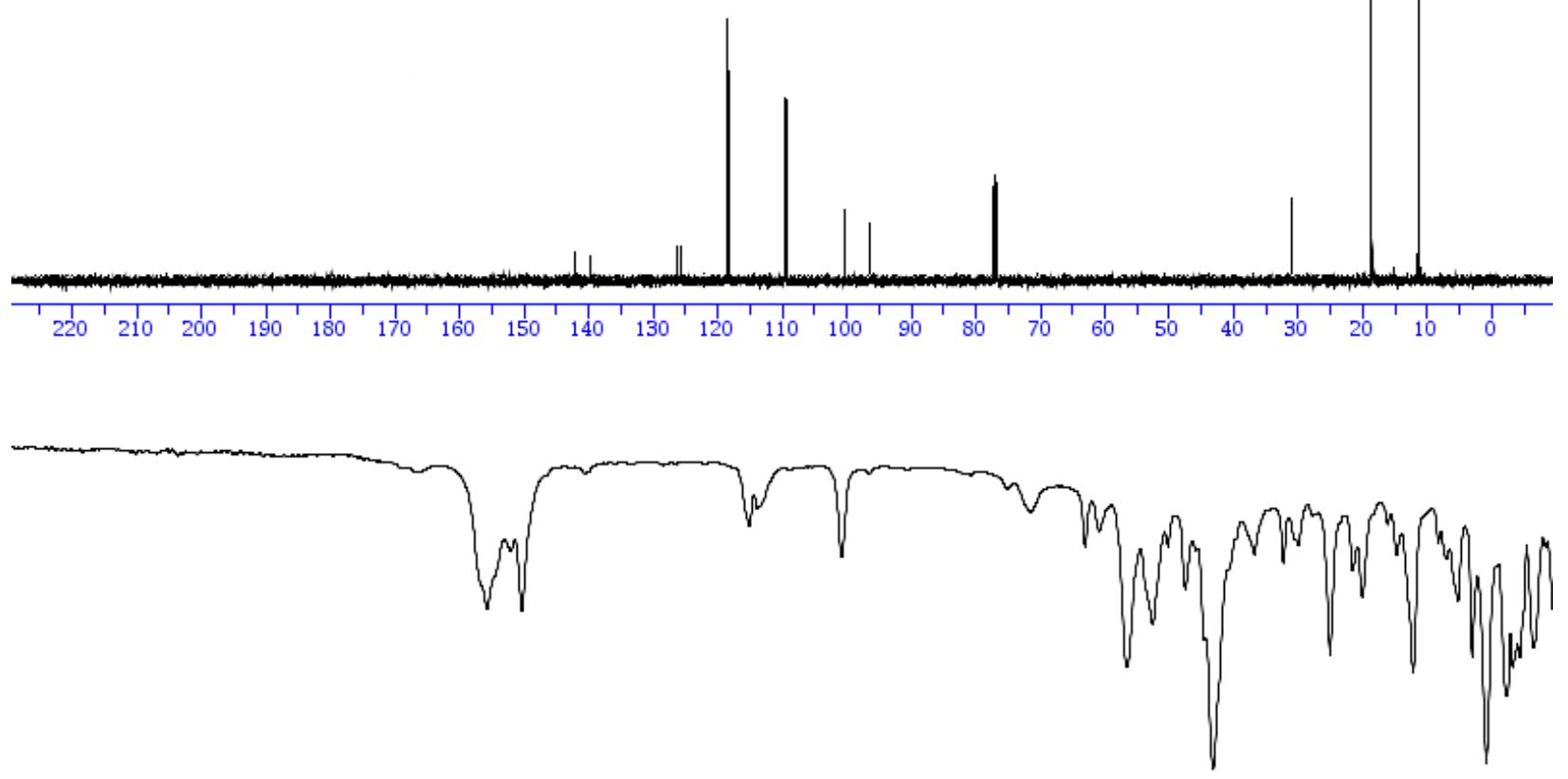

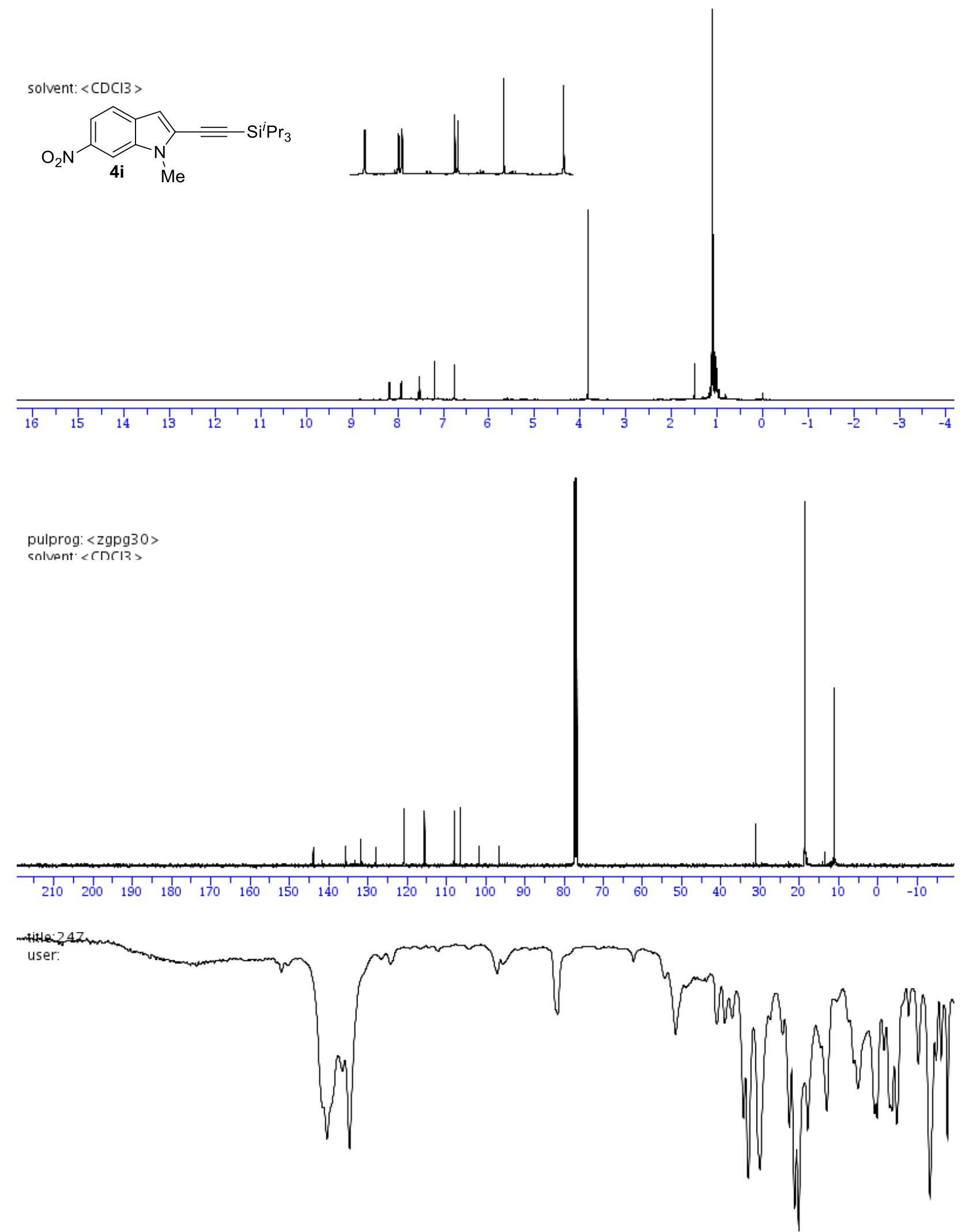

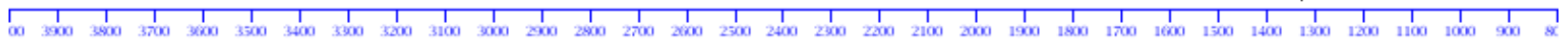


pulprog: $\langle\operatorname{zg} 30\rangle$

solvent: $\langle\mathrm{CDCI}\rangle$
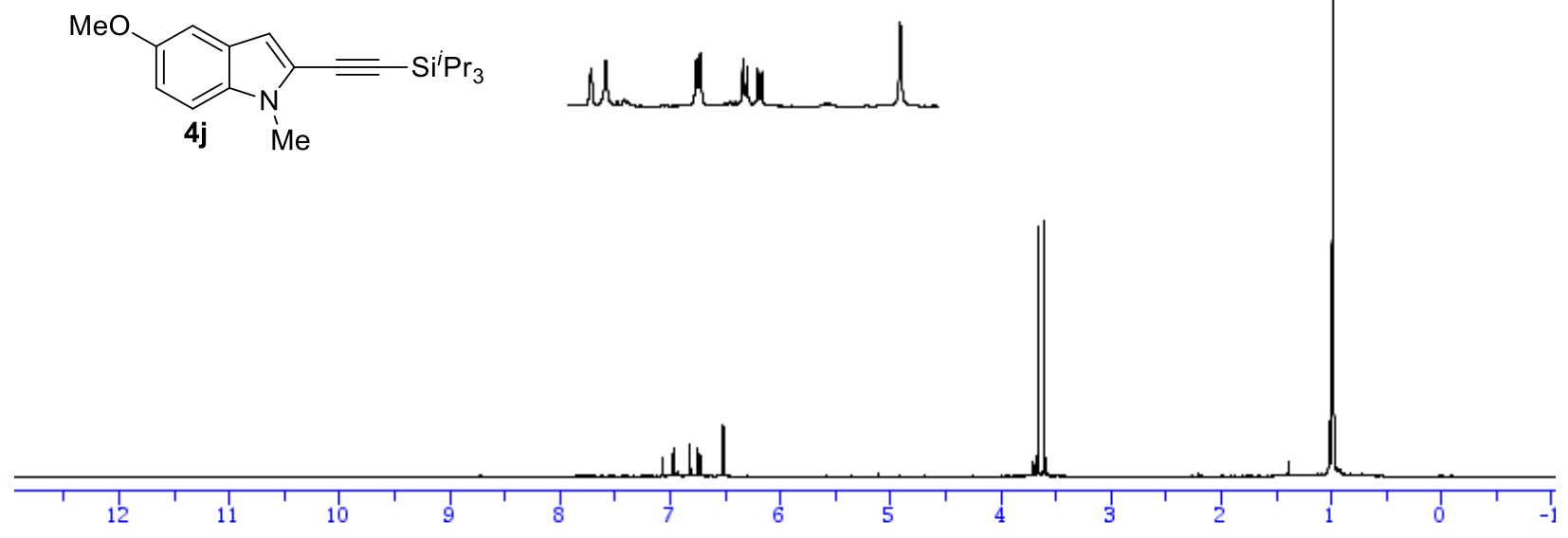

pulprog: $\langle$ zgpg30>

solvent: $\langle\mathrm{CDCl} 3\rangle$
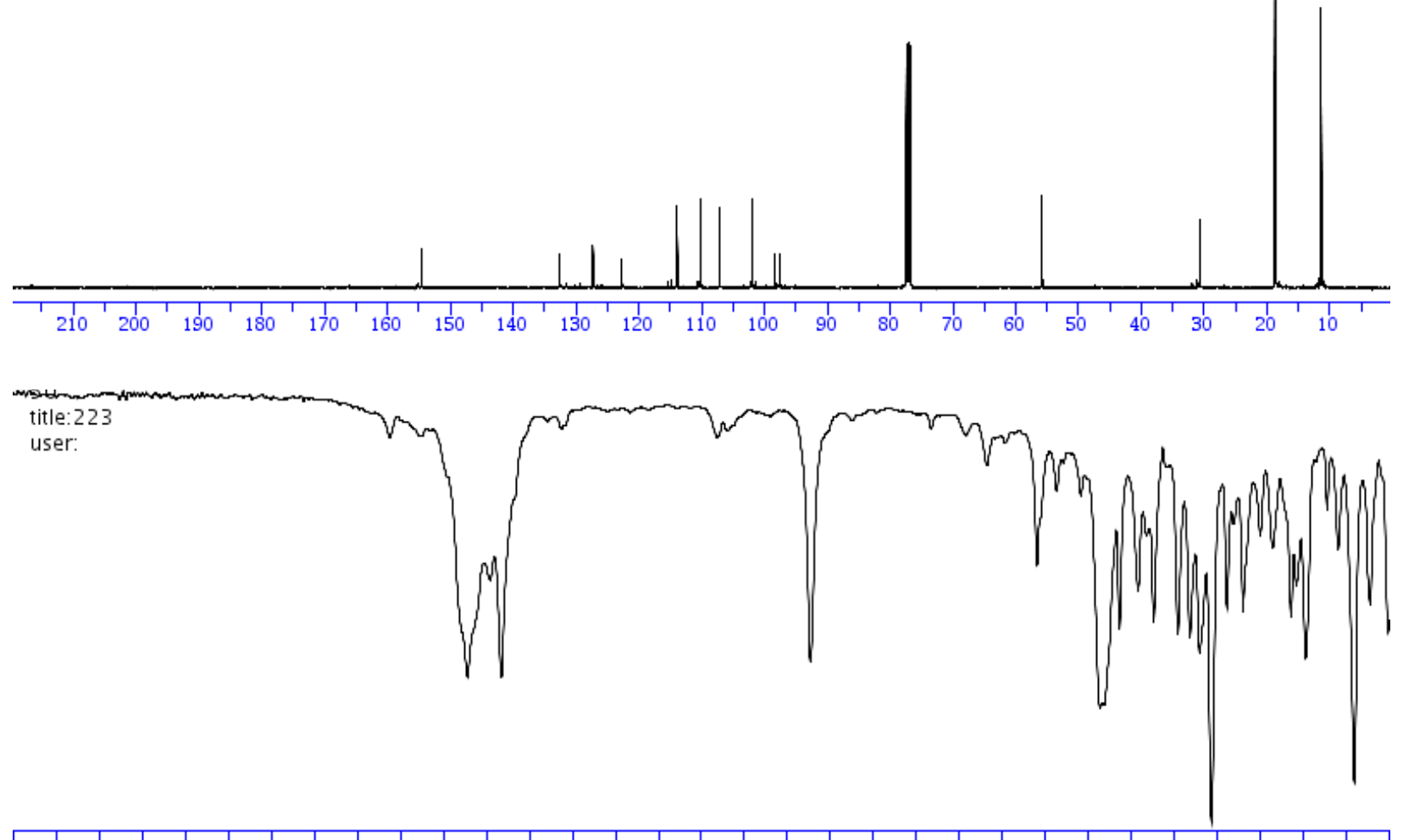

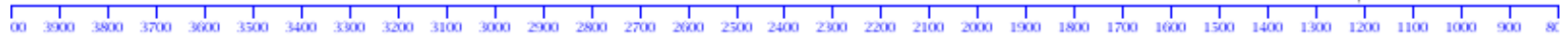


pulprog: $\langle z \mathrm{~g} 30\rangle$

solvent: $\langle\mathrm{CDCl} 3\rangle$
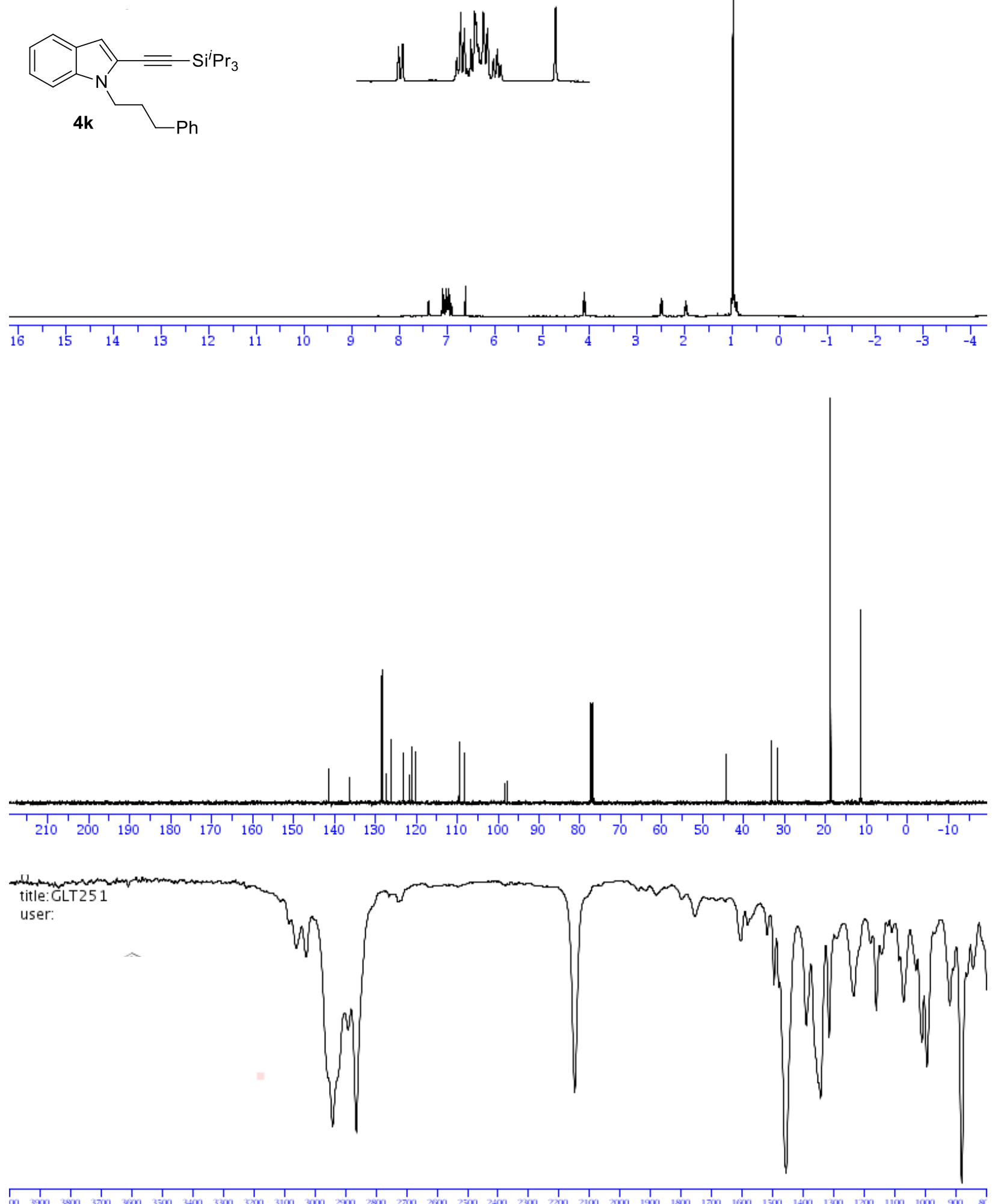

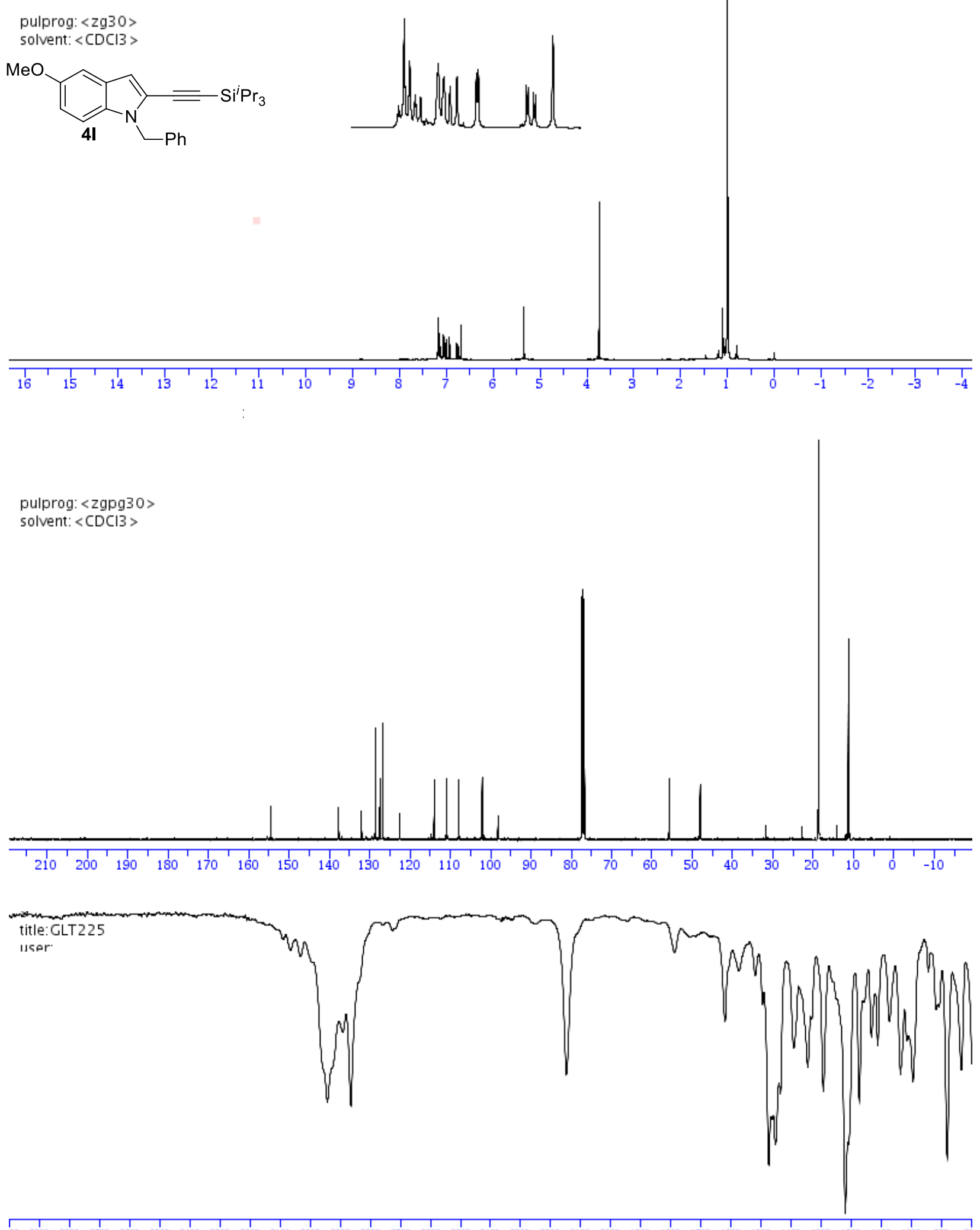
solvent: $\langle\mathrm{CDCI}\rangle$

NS: 2<smiles>CC(C)[SiH2]C#Cc1cc2ccccc2n1Cc1ccccc1</smiles>
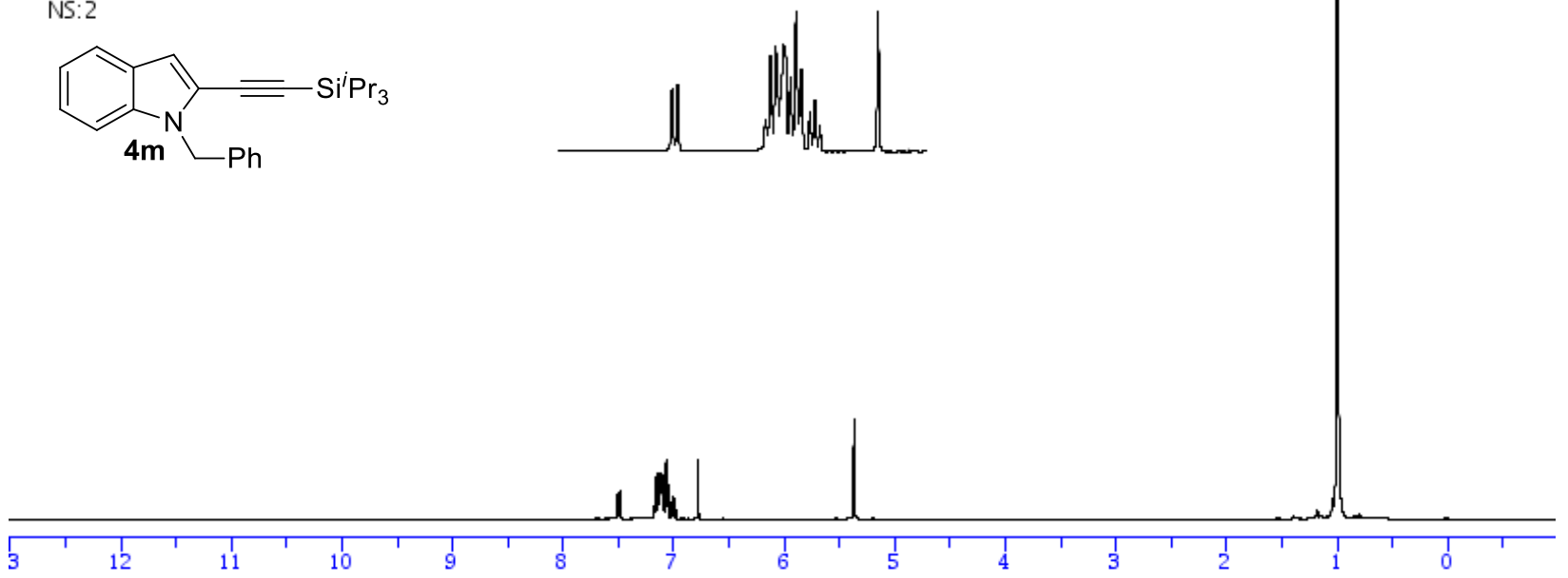

pulprog: <zgpg30 >

solvent: $\langle\mathrm{CDCl} 3\rangle$
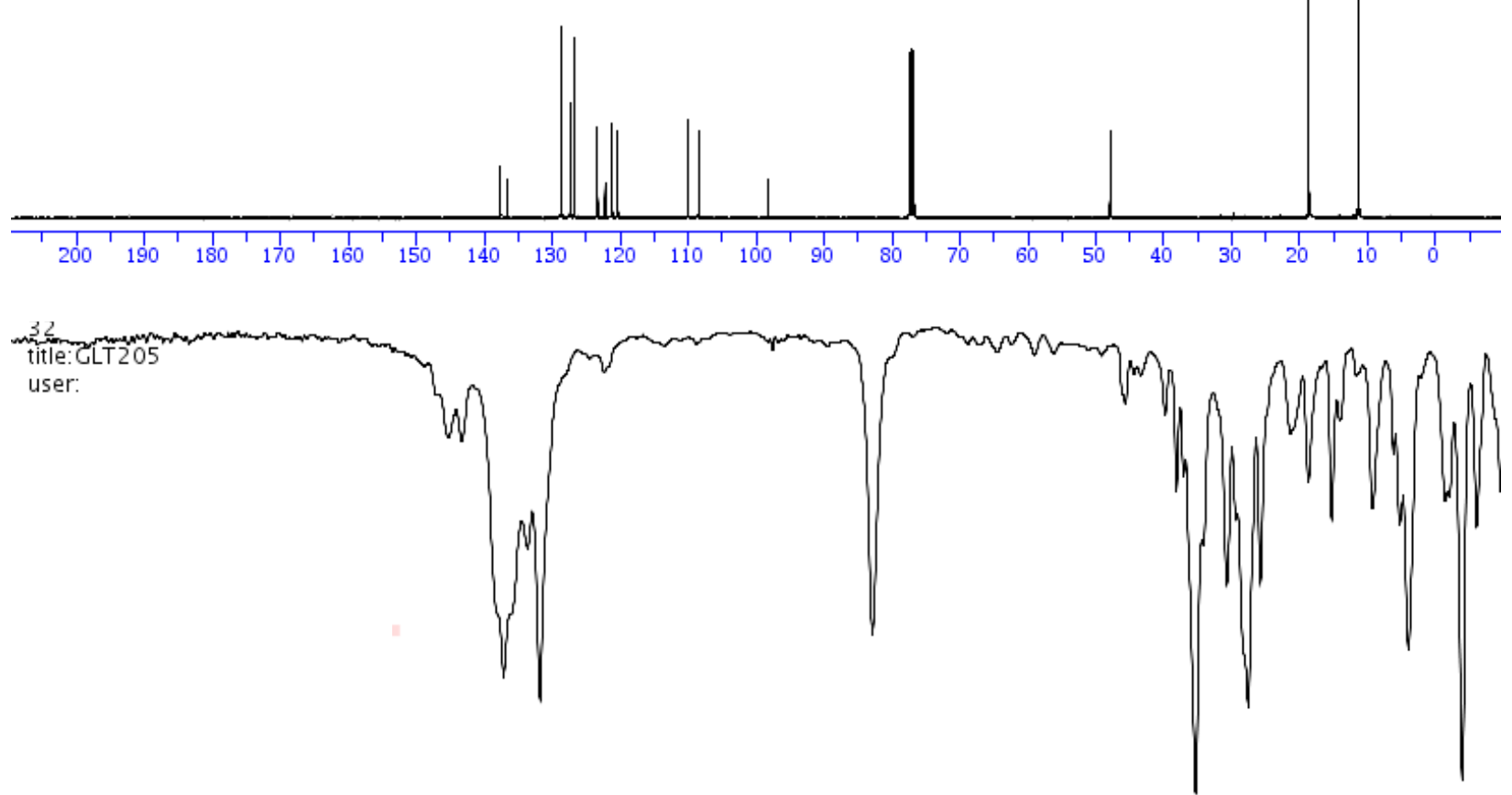

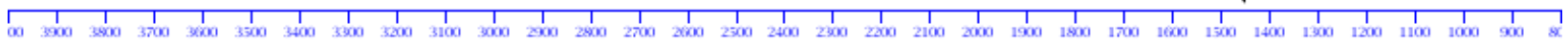



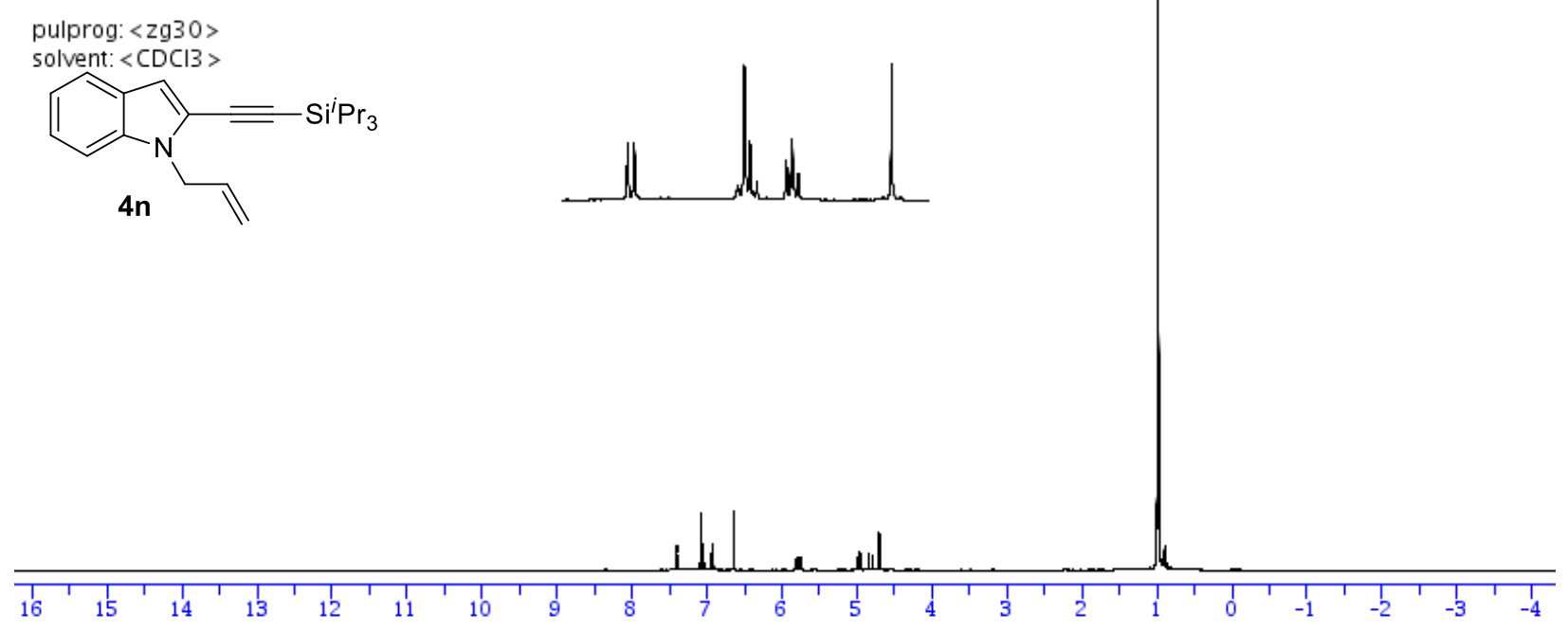

pulprog: $\langle$ zgpg30>

solvent: $\langle\mathrm{CDCl} 3\rangle$
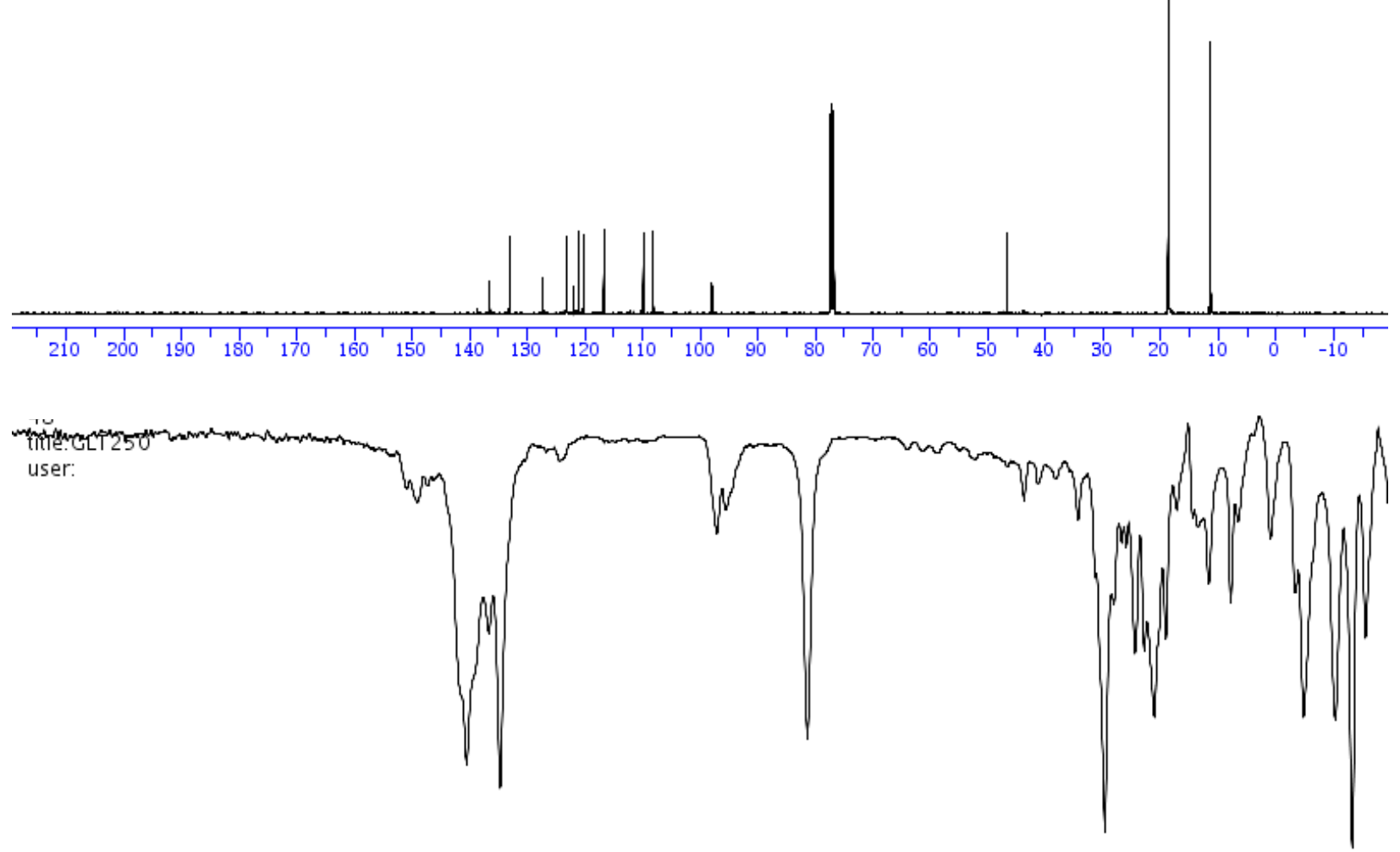

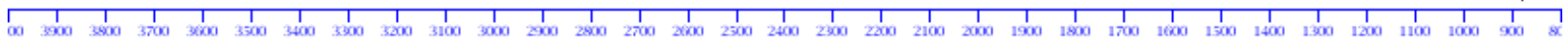


pulprog: $\langle\mathrm{zg} 30\rangle$

solvent: $\langle\mathrm{CDCI} 3\rangle$<smiles></smiles>

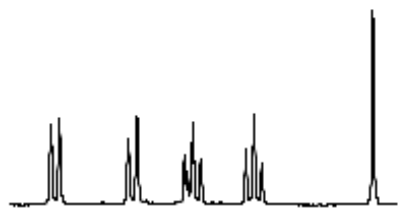

Iive

U

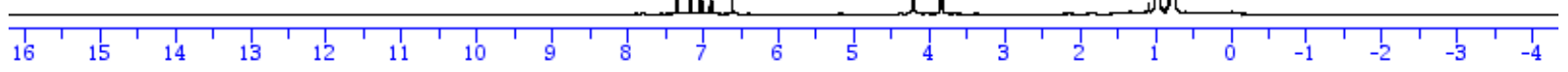

pulprog: <zgpg30>

solvent: $\langle\mathrm{CDCl} 3\rangle$
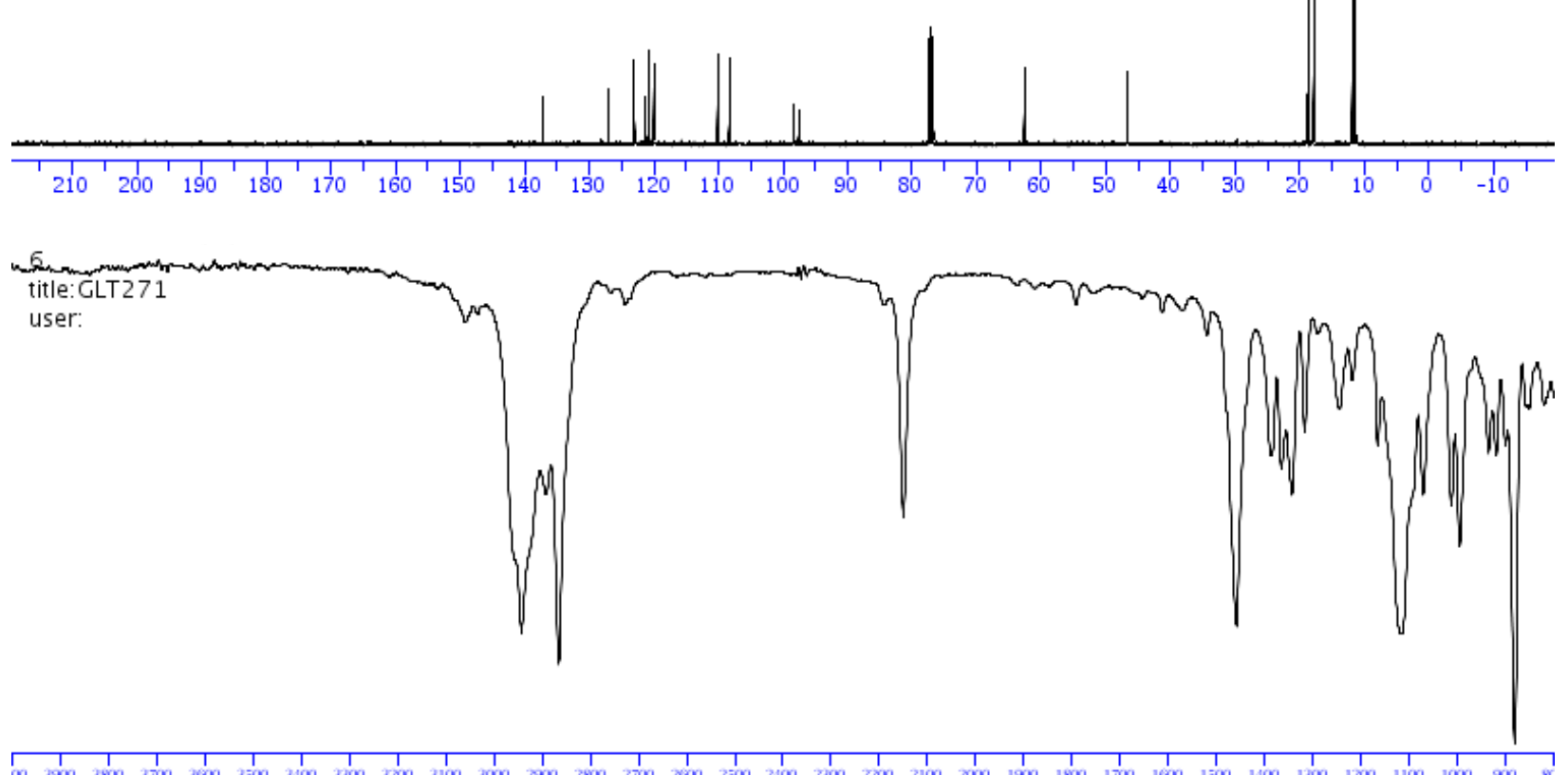

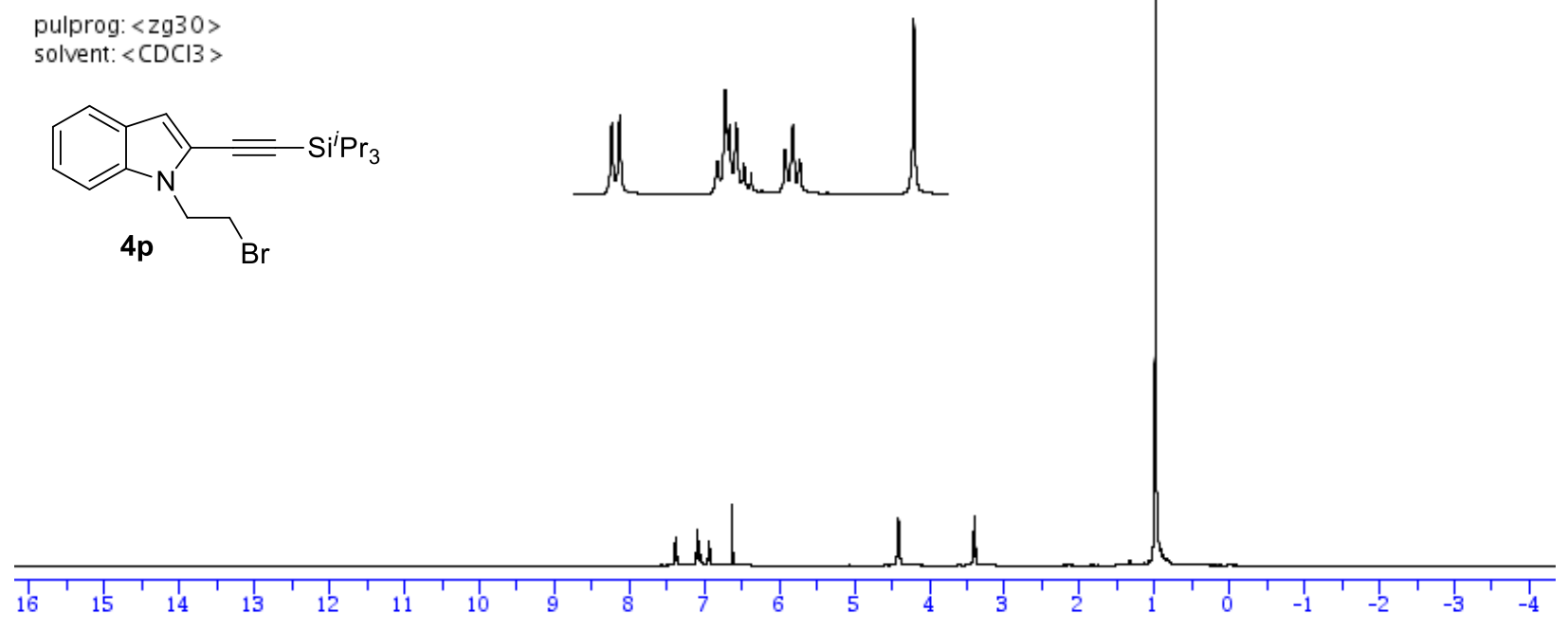

pulprog: <zgpg30>

solvent: $\langle\mathrm{CDCl} 3\rangle$
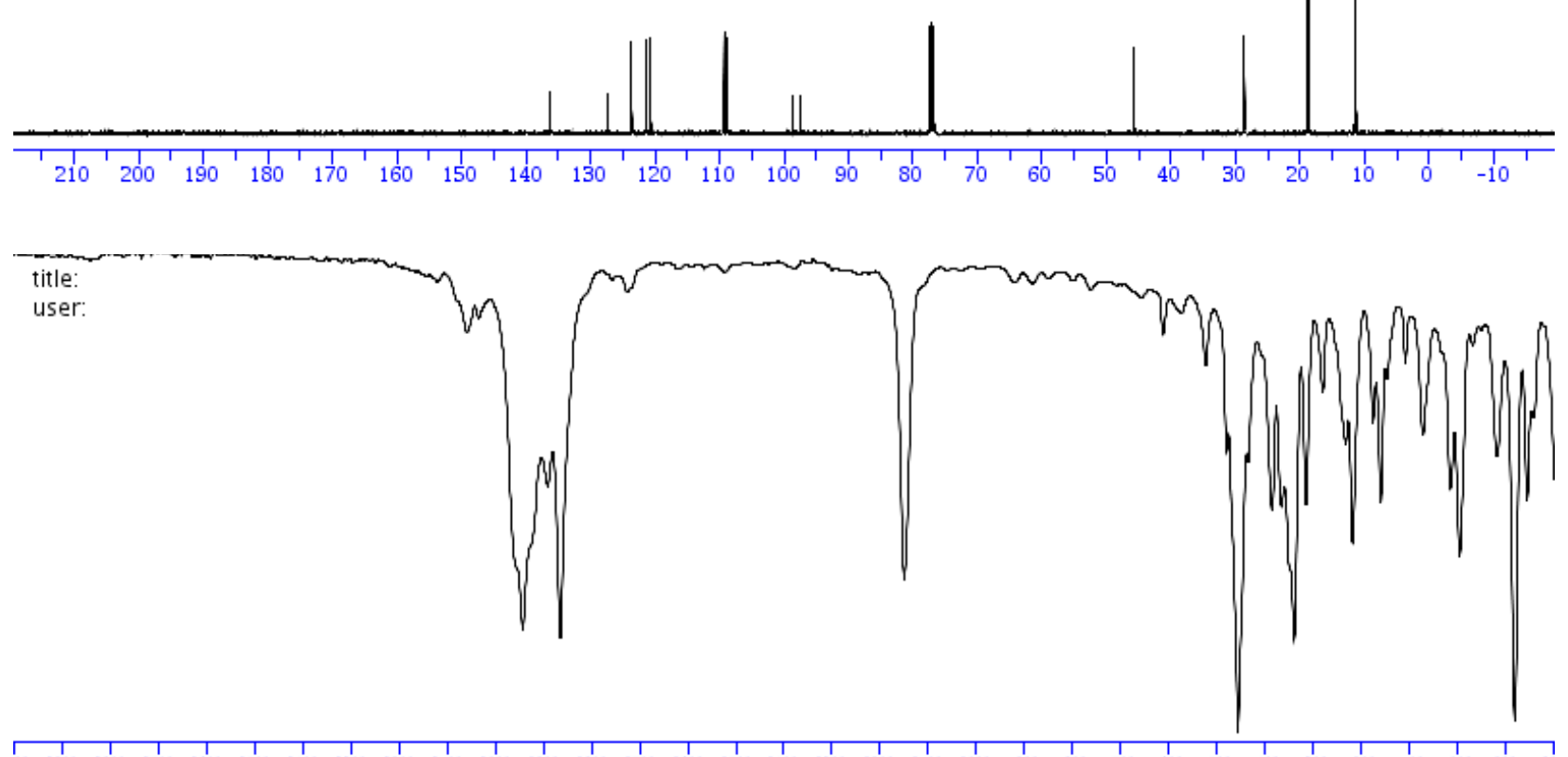

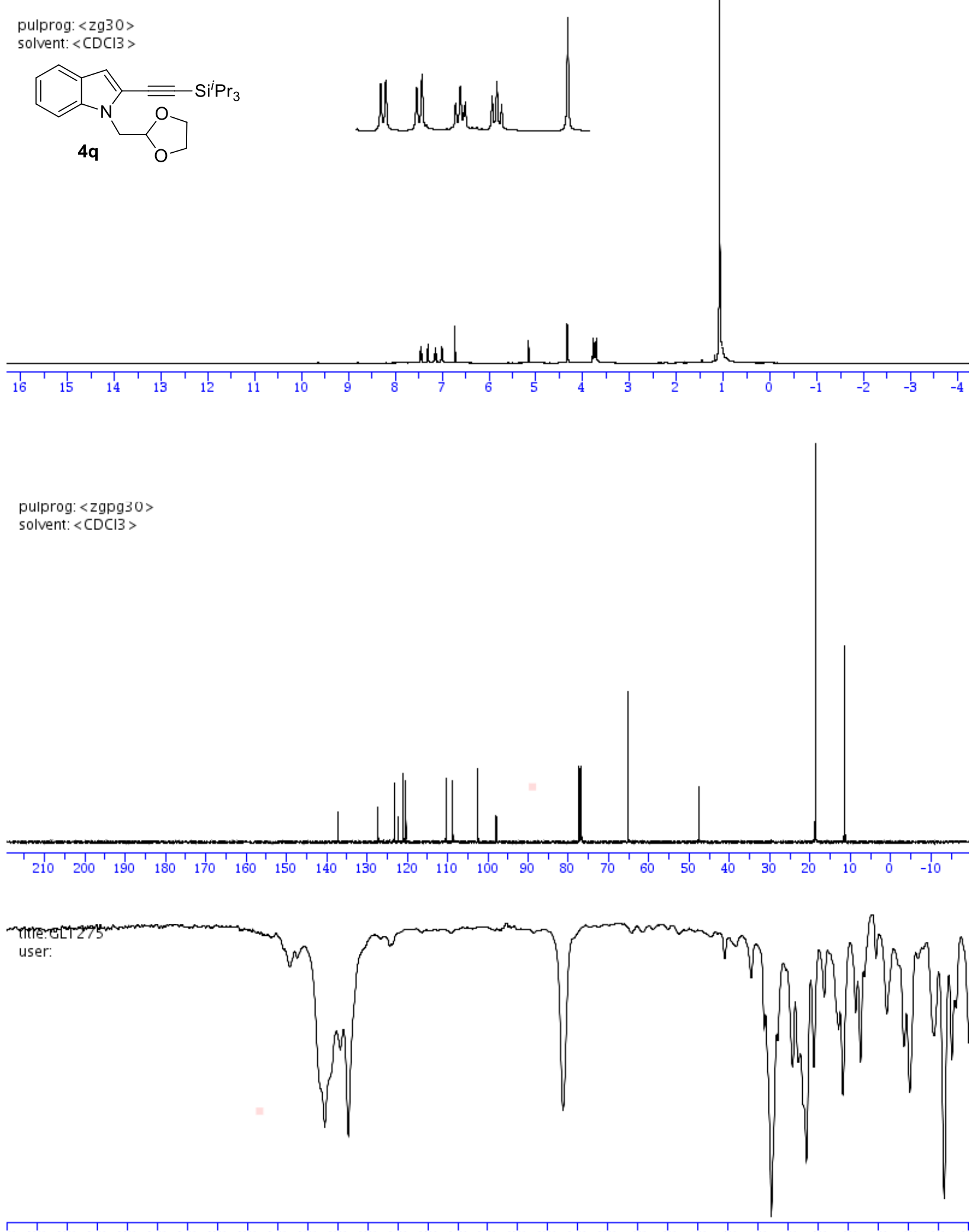